\title{
Singularidades simples de curvas determinantais
}

\author{
Nancy Carolina Chachapoyas Siesquén
}





\title{
Singularidades simples de curvas determinantais ${ }^{1}$
}

\section{Nancy Carolina Chachapoyas Siesquén}

Orientadora: Profa. Dra. Maria Aparecida Soares Ruas

Dissertação apresentada ao Instituto de Ciências Matemáticas e de Computação - ICMC-USP, como parte dos requisitos para obtenção do título de Mestre em Ciências - Matemática.

\author{
USP - São Carlos \\ Julho / 2010
}

\footnotetext{
${ }^{1}$ Este trabalho teve suporte financeiro da CAPES
} 
A Matemática é o mais maravilhoso instrumento criado pelo génio do homem para a descoberta da verdade.

Laisant. 


\section{Agradecimentos}

Gostaria de expressar aqui minha gratidão em primeiro lugar a Deus com todo meu coração por seu infinito amor e pelo grande dom da Vida, que me permitiram chegar onde estou.

Quero expressar meus mais sinceros agradecimentos a minha mãe, María Yolanda, pelo seu carinho e apoio incondicional durante o transcurso da minha vida. A meu pai, Santiago, pelos ensinamentos em minha infância que me foram muito úteis. A meus irmãos: Jimmy, Sandra, José e Alexis pela compreensão, companherismo e carinho durante estes anos. Talvez nem saibam a sua importância em minha vida, meus sobrinhos Jhon, Juliana e Estrella. Alegrias incomparáveis ao lado deles.

À minha orientadora Maria Aparecida Soares Ruas pela amizade, paciência e excelente orientação. Pelas conversas que sempre me esclareciam e acalmavam. Pelo constante incentivo, pela confiança desprendida, acreditando sempre no meu trabalho. Pela enorme contribuição, sem a qual este trabalho não teria sido realizado.

Aos professores do ICMC, com os quais muito aprendi. A Lizandro Sanchez Challapa, a todos os colegas da USP e em especial, àqueles que se fizeram mais presentes: Manuel Zuloeta, Luis Espinoza, Napoleón Caro. A meu amor José Bravo, pelo apoio e pela compreensão com que sempre me brinda.

À Universidade de São Paulo e em especial ao ICMC/USP pelo dinamismo e competência como instituição, e a todos seus funcionários. Aos antigos e inesquecíveis amigos e irmãos de que sentimos saudades, que apesar de estarem em caminhos divergentes, nos são caros e pelos quais temos especial gratidão; em especial para: Rosa Fuentes, Ruth Flores, Jaime Vargas.

A Capes, Coordenação de Aperfeiçoamento de Pessoal de Nível Superior, pelo apoio financeiro. Reitero aqui minhas palavras, sendo este um grande sonho, de que não se pode empreendê-lo sem a ajuda dos que nos cercam e querem bem. Um muito obrigado a todos, por estarem lado a lado nessa caminhada. 
Com amor, a minha mãe Yolanda e a meus irmãos. 


\section{Resumo}

Neste trabalho, estudamos a classificação de singularidades de curvas espaciais simples que não são intersecções completas. O Teorema de Hilbert-Burch nos permite usar a matriz de representação para estudar a variedade definida pelo ideal gerado por seus menores maximais. Da mesma forma, as deformações da variedade determinantal podem ser representadas por perturbações da matriz e qualquer perturbação da matriz fornece uma deformação da variedade. Assim, o estudo das singularidades de curvas determinantais pode ser formulado em termos da matriz de representação da curva. 
viii 


\section{Abstract}

In this work, we study the classification of simple space curve singularities which are not complete intersections. The Theorem of Hilbert-Burch enables us to deal with the presentation matrices instead of the ideals defined by their maximal minors. In the same way, deformations of the determinantal variety can be represented by perturbations of the matrix and any perturbation of the matrix gives rise to a deformation of the variety. Therefore, the study of determinantal curves can be formulated in terms of the presentation matrices. 


\section{Sumário}

Lista de Tabelas $\quad$ xiv

Introdução 1

História . . . . . . . . . . . . . . . . . . . . . . . 2

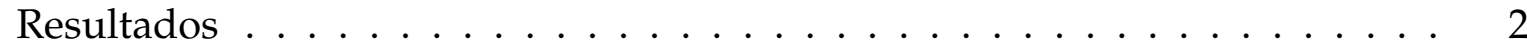

1 Definições e Resultados Básicos 5

1.1 Germes e Jatos . . . . . . . . . . . . . . . . . . . . . . . 5

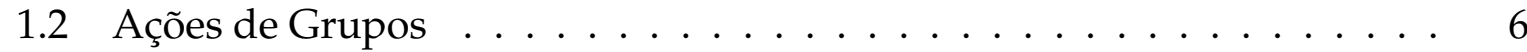

1.3 Germes Finitamente Determinados . . . . . . . . . . . . . . . . . . . . . . 9

1.4 Deformações de Singularidades . . . . . . . . . . . . . . . . . . . 12

1.5 A Profundidade e a Codimensão de um Ideal . . . . . . . . . . . . . . . . 15

1.6 Teorema de Hilbert-Burch . . . . . . . . . . . . . . . . . . . . . . 17

2 Germes Cohen-Macaulay de Codimensão 2 em $\left(\mathbb{C}^{m}, 0\right)$

2.1 Descrição da Matriz de Representação . . . . . . . . . . . . . . . . . . . 21

2.2 O Grupo $\mathcal{G}(n) \ldots \ldots \ldots \ldots \ldots \ldots$

2.3 Espaço Tangente . . . . . . . . . . . . . . . . . . . . 28

2.4 O Cálculo de $T^{1} \ldots \ldots \ldots \ldots \ldots \ldots$

2.5 Determinação Finita da $\mathcal{G}(n)$ - Equivalência $\ldots \ldots \ldots$. . . . . . 36

2.6 Matrizes Quase- Homogêneas . . . . . . . . . . . . . . . . . . . . . 51

3 Classificação de Curvas Espaciais Simples $\quad 57$

3.1 Redução ao Problema . . . . . . . . . . . . . . . . . . . . . . 57

3.2 Germes Simples . . . . . . . . . . . . . . . . . . . . . . . . 59

3.3 Candidatos para Curvas Espaciais Simples . . . . . . . . . . . . . 63 
3.4 Passo I: Classificação de Três Pontos em $\mathbb{P}^{2}$.

3.5 Passo II: Classificação dos 1-Jatos da Matriz de Representação. . . . . . . 67

3.6 Passo III: Procurar Candidatos em cada um dos Casos do Item (2). . . . . 81

3.7 Deformações Semi-Universais das Formas Normais $\ldots \ldots \ldots \ldots \ldots$. . . 87

\begin{tabular}{ll}
\hline Referências Bibliográficas & 95
\end{tabular} 


\section{Lista de Tabelas}

$3.1 \quad$ Curvas espaciais não simples . . . . . . . . . . . . . . . . . . . . . 62

3.2 Curvas espaciais de mutiplicidade $3 \ldots \ldots$. . . . . . . . . . . 64

3.3 Curvas espaciais de mutiplicidade $4 \ldots \ldots$. . . . . . . . . . 65

3.4 Diagrama de adjacências . . . . . . . . . . . . . . . . 91 



\section{Introdução}

Este trabalho tem o objetivo de estudar curvas espaciais simples que não são necessariamente intersecções completas. Nosso estudo se baseia no trabalho de Anne Frühbis-Krüger [15], que classifica o espaço de curvas determinantais através de uma relação de equivalência definida pelo grupo $\mathcal{G}(n)$, que é um subgrupo do grupo de contato. Tais curvas podem ser representadas como a variedade do ideal gerado pelos menores maximais de uma matriz $3 \times 2$, se constituindo numa variedade 1 - dimensional Cohen-Macaulay de codimensão 2.

O trabalho [15] complementa a classificação de curvas que são intersecções completas obtida por M. Giusti em seu artigo [20].

O Teorema de Hilbert-Burch afirma que toda variedade Cohen-Macaulay de codimensão dois é obtida pelos menores maximais de uma matriz de representação $n \times$ $(n+1), M$, e viceversa. No mesmo contexto toda deformação de tal variedade pode ser representada por perturbações da matriz $M$, o que motiva a utilização da teoria de singularidades de matrizes para estudar estas variedades.

Este resultado permite reformular o espaço normal, $N_{X}$, e o espaço das deformações de primeira ordem, $T_{X}^{1}$, em termos da matriz representação. A ação do grupo $\mathcal{G}(n)$ é dada por mudanças de coordenadas na fonte e transformações de linhas e colunas da matriz. A relação de equivalência do grupo $\mathcal{G}(n)$ para matrizes se identifica com a relação de equivalência do grupo $\mathcal{K}$ para funções determinadas pelos seus menores maximais. Uma singularidade é simples se e só se pode se deformar a um número finito de classes de equivalência, dada pelo grupo $\mathcal{G}(n)$.

Neste trabalho, desenvolvemos o estudo da $\mathcal{G}(n)$ - equivalência e aplicamos os resultados da teoria de singularidades de matrizes para obter a classificação das singularidades simples de curvas determinantais.

No Capítulo 1, relembramos algumas definições e resultados básicos tais como germes de aplicações, ações de grupos, determinação finita e deformações de singularidades. Além disso, apresentaremos os conceitos e propriedades de anéis Cohen- 
Macaulay e o Teorema de Hilbert-Burch.

No Capítulo 2, usamos o teorema de Hilbert-Burch para obter uma boa descrição de uma variedades Cohen-Macaulay de codimensão dois e de suas deformações em termos da matriz de representação, introduzimos a $\mathcal{G}(n)$ equivalência, definimos o espaço tangente e estudamos a $\mathcal{G}(n)$ - determinação finita em termos da matriz de representação. Apresentaremos duas provas do critério infinitesimal de determinação finita baseadas nos trabalhos de [15] e [27]. Além disso estudaremos matrizes quasehomogêneas.

No Capítulo 3, definimos a noção de germes simples e estudaremos critérios que os identifiquem. Mostraremos que os valores para $n$ são 1 e 2 . O caso $n=1$, foi estudado por M.Giusti em [20]. Apresentaremos duas Tabelas de curvas simples contendo as classes $A_{k} \vee L, D_{k} \vee L$ e $E_{k} \vee L$. Estas classes são obtidas pela união de uma curva plana de tipo $A_{k}, D_{k}$ ou $E_{k}$ e uma linha não contida nesse plano intersectando à curva plana em seus pontos singulares. Além disso apresentamos o diagrama de adjacências obtidas em [15].

\section{História}

Na teoria de classificação das singularidades, Arnold foi o primeiro a classificar singularidades simples de hipersuperfícies em seu trabalho pioneiro [? ], que contribuiu com as famosas listas de singularidades de tipo ADE. Giusti em seu trabalho [20], alguns anos depois, obteve uma lista de singularidades de intersecções completas simples, e combinando estas duas listas temos uma lista de curvas espaciais simples Cohen-Macaulay de codimensão dois que são intersecções completas. Uma década mais tarde, a lista de Giusti foi completada por Anne Frühbis [15] que contribuiu com uma lista de curvas espaciais simples Cohen-Macaulay de codimensão dois que não são intersecções completas. Além disso, Gibson e Hobbs [19] obtiveram uma classificação do espaço de curvas irredutíveis simples com relação a $\mathcal{A}$-equivalência.

A teoria de curvas tem sido amplamente estudada por diversos autores.

\section{Resultados}

Os resultados mais importantes estudados neste trabalho são mencionados nesta seção e suas provas são encontradas na seção (2.1), página (21) e na seção (3.3).

Considere $\mathcal{P}$ a algebra $\mathbb{K}\left\{x_{1}, \ldots, x_{m}\right\}$ das séries convergentes. 


\section{Proposição 0.1.}

1. Sejam $M \in \operatorname{Mat}_{(n+1) \times n}\left(\mathbb{C}\left\{x_{1}, x_{2}, \cdots, x_{m}\right\}\right), \Delta=\left\langle\Delta_{1}, \Delta_{2}, \cdots, \Delta_{n+1}\right\rangle$ o ideal gerado por seus menores maximais (o indice de $\Delta_{i}$ indica o indice da linha de $M$ que foi eliminada para calcular o menor).

Se a codim $(V(\Delta)) \geq 2$, a seguinte seqüência

$$
0 \longrightarrow \mathcal{P}^{n} \stackrel{M}{\longrightarrow} \mathcal{P}^{n+1} \stackrel{\Delta}{\longrightarrow} \mathcal{P} \longrightarrow \frac{\mathcal{P}}{(\Delta)} \longrightarrow 0
$$

é exata. Além disso $\frac{\mathcal{P}}{(\Delta)}$ é Cohen-Macaulay e Codim $(V(\Delta))=2$.

2. Se $X=V(I) \subseteq \mathbb{C}^{m}$ é Cohen-Macaulay, $\operatorname{codim}(X)=2$ então $\mathcal{P} / I$ tem uma resolução livre minimal do tipo.

$$
0 \longrightarrow \mathcal{P}^{n} \stackrel{M}{\longrightarrow} \mathcal{P}^{n+1} \stackrel{I}{\longrightarrow} \mathcal{P} \longrightarrow \frac{\mathcal{P}}{I} \longrightarrow 0
$$

Além disso existe uma unidade $u \in \mathcal{P}$ tal que $I=u . \Delta$, onde $\Delta=\left\langle\Delta_{1}, \ldots, \Delta_{n+1}\right\rangle$.

3. Qualquer perturbação da $M$ dá origem a uma deformação de $X$.

4. Qualquer deformação da variedade X pode ser gerada por uma perturbação da matriz $M$.

Teorema 0.2. (Anne Frühbis-Krüger, [15]) As seguintes singularidades são curvas espaciais simples de multiplicidade 3:
1. $\left(\begin{array}{ccc}z & y & x^{k-3} \\ 0 & x & y\end{array}\right), A_{k-3} \vee L \quad k \geq 4$
4. $\left(\begin{array}{ccc}z & y & x^{3} \\ x & z & y\end{array}\right), E_{8}(1)$
2. $\left(\begin{array}{ccc}z & y & x^{2} \\ x & z & y\end{array}\right), E_{6}(1)$
5. $\left(\begin{array}{ccc}z+x^{2} & y & x^{k+2} \\ 0 & z & y\end{array}\right), J_{2, k}(2)$
3. $\left(\begin{array}{ccc}z+x^{2} & y & x \\ 0 & z & y\end{array}\right), E_{7}(1)$
6. $\left(\begin{array}{ccc}z & y & x^{3} \\ x^{2} & z & y\end{array}\right), E_{12}(2)$

Teorema 0.3. (Anne Frühbis-Krüger, [15]) As seguintes singularidades são curvas espaciais simples de multiplicidade 4:
1. $\left(\begin{array}{ccc}z & 0 & x^{k+2}-y^{2} \\ 0 & x & y\end{array}\right), D_{k+4} \vee L$
2. $\left(\begin{array}{ccc}z & -y^{2} & -x^{3} \\ 0 & x & y\end{array}\right), E_{6} \vee L$ 

3. $\left(\begin{array}{ccc}z & x^{3}-y^{2} & 0 \\ 0 & x & y\end{array}\right), E_{7} \vee L$
6. $\left(\begin{array}{ccc}z & x & y \\ 0 & y & x^{2}-z^{3}\end{array}\right), T_{7}^{*}$
4. $\left(\begin{array}{ccc}z & -y^{2} & -x^{4} \\ 0 & x & y\end{array}\right), E_{8} \vee L$
7. $\left(\begin{array}{ccc}z & x y & x^{2} \\ x & z & y\end{array}\right), U_{7}^{*}$
5. $\left(\begin{array}{ccc}z & x & y \\ 0 & y & x^{2}-z^{2}\end{array}\right), S_{6}^{*}$
8. $\left(\begin{array}{ccc}z & y^{2} & x^{2} \\ x & z & y\end{array}\right), W_{8}^{*}$ 


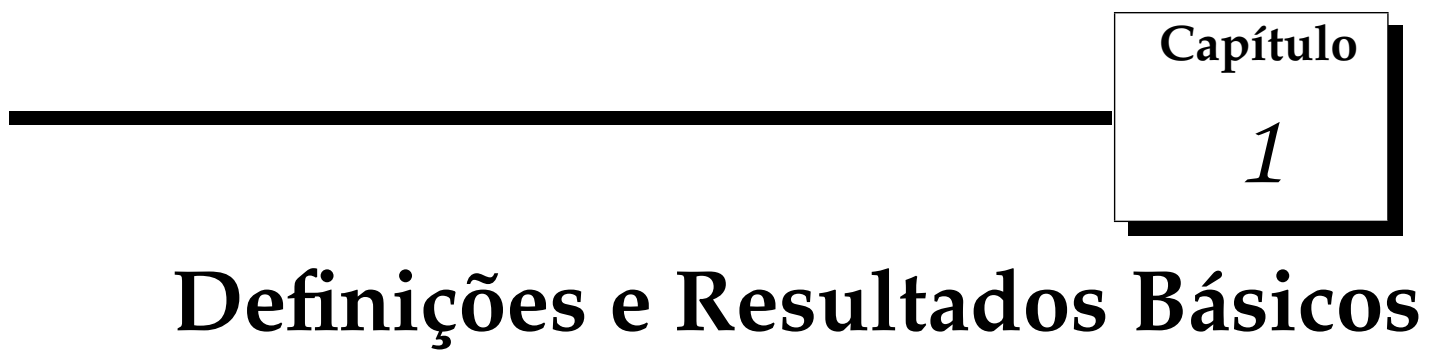

Neste capítulo apresentamos as definições e principais resultados da teoria de singularidades que serão usados para o desenvolvimento deste trabalho . As principais referências para este capítulo são [18], [30], [21], [14], [31], [3].

\subsection{Germes e Jatos}

Sejam $N$ e $P$ variedades de dimensões $n$ e $p$ respectivamente, e $x \in N$. No conjunto das aplicações suaves numa vizinhança de $x$ em $N$ e com valores em $P$ introduzimos a seguinte relação de equivalência $(\sim)$ :

Definição 1.1. Duas aplicações suaves $f_{1}: U_{1} \rightarrow P$ e $f_{2}: U_{2} \rightarrow P$ são equivalentes, $\left(f_{1} \sim f_{2}\right)$, quando existir uma vizinhança $U \subset U_{1} \cap U_{2}$ de $x$ em $N$ tal que as restrições $\left.f_{1}\right|_{U}$ e $\left.f_{2}\right|_{U}$ coincidem.

As classes de equivalência dessa relação são chamadas de germes de aplicações em $x$ e um elemento da classe de equivalência é chamado de representante do germe. Notação: $f:(N, x) \rightarrow(P, y), f(x)=y$ (chamamos $x$ de fonte e $y$ de meta do germe). Para cada germe $f:(N, x) \rightarrow(P, y)$ temos a derivada $d_{x} f: T_{x} N \rightarrow T_{y} P$ que é definida como sendo a derivada em $x$ de qualquer representante. Dizemos que $f:(N, x) \rightarrow$ $(P, y)$ é um germe de difeomorfismo se, e somente se, um de seus representantes (e portanto qualquer) é um difeomorfismo local, e portanto, segue do teorema da função inversa que sua derivada é um isomorfismo. O posto de um germe é definido como o posto de sua derivada em $x$. Quando o posto é igual à dimensão de $N$ o germe é imersível e quando o posto é igual à dimensão de $P$ o germe é submersível. Quando o germe não é imersível e nem submersível em $x$ dizemos que $x$ é ponto singular. 
Definição 1.2. Dois germes $f_{1}:\left(N_{1}, x_{1}\right) \longrightarrow\left(P_{1}, y_{1}\right)$ e $f_{2}:\left(N_{2}, x_{2}\right) \longrightarrow\left(P_{2}, y_{2}\right)$ são $\mathcal{A}$ equivalentes quando existem germes de difeomorfismos $h:\left(N_{2}, x_{2}\right) \rightarrow\left(N_{1}, x_{1}\right)$ e $k$ : $\left(P_{2}, y_{2}\right) \rightarrow\left(P_{1}, y_{1}\right)$ para os quais o seguinte diagrama comuta:

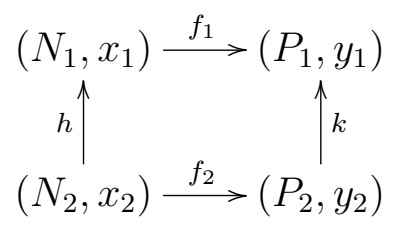

isto é $f_{1}=k \circ f_{2} \circ h^{-1}\left(\right.$ ou $\left.f_{1} \circ h=k \circ f_{2}\right)$.

Como qualquer germe $f:(N, x) \rightarrow(P, y)$ é equivalente a algum germe $\left(\mathbb{R}^{n}, 0\right) \rightarrow\left(\mathbb{R}^{p}, 0\right)$, consideramos apenas germes $\left(\mathbb{R}^{n}, 0\right) \rightarrow\left(\mathbb{R}^{p}, 0\right)$.

Definição 1.3. (O espaço dos jatos $J_{k}(n, p)$ ) É o espaço vetorial real das aplicações $f: \mathbb{R}^{n} \rightarrow$ $\mathbb{R}^{p}$ onde cada componente $f_{i}$ de $f$ é um polinômio de grau $\leq k$ nas coordenadas canônicas $x_{1}, x_{2}, \cdots, x_{n}$ de $\mathbb{R}^{n}$ com termo constante nulo. Os elementos de $J_{k}(n, p)$ são chamados de $k$-jatos.

Para cada aplicação de classe $C^{\infty} f: \mathbb{R}^{n} \rightarrow \mathbb{R}^{p}$ e cada $a \in \mathbb{R}^{n}$, definimos a aplicação $j^{k} f: \mathbb{R}^{n} \rightarrow J_{k}(n, p)$ por : $j^{k} f(a)$ é o polinômio de Taylor de $f(x+a)-f(a)$ de ordem $k$ na origem. A aplicação $j^{k} f$ é de classe $C^{\infty}$ e $j^{k} f(a)$ é chamado o $k$-jato de $f$ em $a$.

Exemplo 1.4. Seja $f: \mathbb{R} \rightarrow \mathbb{R}$ então $j^{k} f(a)=f^{\prime}(a) x+\frac{f^{\prime \prime}(a)}{2 !} x^{2}+\cdots+\frac{f^{k}(a)}{k !} x^{k}$

Definição 1.5. Dois germes de aplicações $f$ e $g$ de classe $C^{\infty}$ têm d-jatos $A^{d}$ equivalentes se existem germes de difeomorfismos $h:\left(\mathbb{R}^{n}, 0\right) \rightarrow\left(\mathbb{R}^{n}, 0\right)$ e $k:\left(\mathbb{R}^{p}, 0\right) \rightarrow\left(\mathbb{R}^{p}, 0\right)$ tais que:

$$
j^{d}(f \circ h)=j^{d}(k \circ d)
$$

Esta relação induz naturalmente uma relação de equivalência nos espaço dos jatos $J^{d}(n, p)$.

\subsection{Ações de Grupos}

Definição 1.6. (Ações de grupos sobre um conjunto) Seja G um grupo e M um conjunto. Uma ação de grupos de G sobre M é uma aplicação,

$$
\begin{aligned}
\Psi: G \times M & \longrightarrow M \\
(g, x) & \longmapsto g . x
\end{aligned}
$$

satisfazendo as seguintes propriedades: 
- $1 . x=x, \forall x \in M$, onde 1 é a identidade em $G$

- $(g h) . x=g .(h . x) \forall x \in M, \forall g, h \in G$.

Definimos a órbita de $x$, para qualquer $x \in M$ :

$$
G \cdot x=\{g \cdot x / g \in G\} .
$$

Dizemos que $x, y \in M$ são $G$ - equivalentes se pertencem à mesma órbita por essa ação.

Definição 1.7. Grupo de Lie. Um grupo de Lie é um grupo e uma variedade diferenciável onde as operações de grupo (a multiplicação e a inversão) são aplicações de classe $C^{\infty}$.

Seja $\mathbb{K}$ o corpo dos números reais ou complexos. Indicamos por $\mathcal{O}_{n}$ o conjunto de germes de funções analíticas $\left(\mathbb{K}^{n}, 0\right) \rightarrow \mathbb{K}$. Usando operações de soma e multiplicação de $\mathbb{K}$ temos que $\mathcal{O}_{n}$ possui uma estrutura de anel comutativo com unidade. Este anel é um anel local (possui um único ideal maximal), cujo ideal maximal é dado por $\mathbf{m}=$ $\left\{f \in \mathcal{O}_{n} / f(0)=0\right\}$. Considere $\mathcal{O}(n, p)=\left\{f: \mathbb{K}^{n}, 0 \rightarrow \mathbb{K}^{p} / \mathrm{f}\right.$ é um germe analítico $\}$ como um $\mathcal{O}_{n}$ módulo.

Apresentaremos a seguir alguns exemplos de ações de grupos que utilizaremos em nosso trabalho.

Exemplo 1.8. Mudança de coordenadas na fonte. (O grupo $\left.\mathcal{R}_{n}\right)$

Seja $\mathcal{R}_{n}=\left\{h:\left(\mathbb{K}^{n}, 0\right) \rightarrow\left(\mathbb{K}^{n}, 0\right) / h\right.$ é um germe de difeomorfismo $\}$. Defina-se:

$$
\begin{aligned}
\Psi: \mathcal{R}_{n} \times \mathbf{m}_{n} \mathcal{O}(n, p) & \longrightarrow \mathbf{m}_{n} \mathcal{O}(n, p) \\
(h, f) & \longmapsto f \circ h^{-1}
\end{aligned}
$$

\section{Exemplo 1.9. Equivalência de contato $\mathcal{K}$}

Seja $\mathcal{K}=\left\{H:\left(\mathbb{K}^{n} \times \mathbb{K}^{p}, 0\right) \rightarrow\left(\mathbb{K}^{n} \times \mathbb{K}^{p}, 0\right) / H\right.$ é um germe de difeomorfismo, $H(x, y)=$ $(h(x), \Theta(x, y))$ onde $h \in \mathcal{R}$ e $\Theta(x, 0)=0$ para $x$ próximo a 0$\}$.

Dois germes $f, g \in \mathbf{m}_{n} \mathcal{O}(n, p)$ são $\mathcal{K}$-equivalentes se existe $H=(h, \Theta) \in \mathcal{K}$ tal que :

$$
(h(x), g \circ h(x))=H(x, f(x))
$$

\section{Exemplo 1.10. C equivalência}

Seja $\mathcal{C}$ o subgrupo de $\mathcal{K}$ definido: $\mathcal{C}=\left\{H \in \mathcal{K} / H=\left(i d_{n}, \Theta\right)\right\}$. Dizemos que dois germes $f, g \in \mathbf{m}_{n} \mathcal{O}(n, p)$ são $\mathcal{C}$-equivalentes se existe $H=\left(i d_{n}, \Theta\right) \in \mathcal{C}$ tal que :

$$
(x, g(x))=H(x, f(x)),
$$


Temos a seguinte proposição que nos permite calcular o espaço tangente à órbita.

Proposição 1.11. Seja $\phi: G \times M \rightarrow M$ uma ação suave de um grupo de Lie G sobre uma variedade suave $M$. Assuma que todas as órbitas são subvariedades suaves de $M$. Então para cada $x \in M$ a aplicação natural: $\phi_{x}: G \longrightarrow G \cdot x$ dada por: $g \longmapsto g . x$ é uma submersão.

Observação 1.12. Segue-se desta proposição que o espaço tangente à órbita é $T_{x}(G . x)=$ $\left(d \phi_{x}\right)_{1}\left(T_{1} G\right)$.

Proposição 1.13. O grupo de contato $\mathcal{K}$ é um produto semi-direto de $\mathcal{R}_{n}$ e $\mathcal{C}$

Demonstração: Identifique $\mathcal{R}=\{h, h \times 1 / h \in \mathcal{R}\}$, logo $\mathcal{R}$ é um subgrupo de $\mathcal{K}$, temos que $\mathcal{K}=\mathcal{R}$.C. De fato, dado $(h, H) \in \mathcal{K}$ podemos escrever $(h, H)=(h, h \times 1) .(1,(h \times$ $\left.1)^{-1} \circ H\right) \in \mathcal{R}_{n} \cdot \mathcal{C}$.

Verifica-se também que $\mathcal{C} \triangleleft \mathcal{K}$ e que $\mathcal{R}_{n} \cap \mathcal{C}=\{i d\}$ e portanto $\exists \Psi: \mathcal{R} \rightarrow \operatorname{Aut}(\mathcal{C}, \mathcal{C})$ tal que $: \mathcal{K} \cong \mathcal{R}_{n} \times_{\Psi} \mathcal{C}$

Lema 1.14. (Lema de Nakayama) Seja $R$ um anel comutativo com identidade 1 e $\mathbf{m}$ um ideal em $R$ com a propriedade que $1+x$ é invertível em $R, \forall x \in \mathbf{m}$.

Seja $C$ um $R$-módulo e $A, B$ sub-módulos de $C$ com A finitamente gerado.

Se $A \subseteq B+\mathbf{m} A$ então $A \subseteq B$.

Lema 1.15. (Lema de Mather) Seja G um grupo de Lie, $M$ uma variedade suave, $\phi: G \times M \rightarrow$ $M$ uma ação. Considere $V \in M$ uma subvariedade conexa de $M$ e suponha que as órbitas são subvariedades suaves de M. Então $V$ está contida numa única órbita se, somente se:

1. $T_{v} V \subseteq T_{v}(G \cdot v) \forall v \in V$.

2. $\operatorname{dim}_{v}(G . v)$ é constante $\forall v \in V$.

Lema 1.16. (Lema de Hadamard) Seja $U$ uma vizinhança conexa de 0 em $\mathbb{R}^{n}$, e seja $f$ uma função suave definida sobre $U \times \mathbb{R}^{q}$ que se anula em $0 \times \mathbb{R}^{q}$, então existem funções suaves $f_{1}, f_{2}, \ldots, f_{n}$ definidas em $U \times \mathbb{R}^{q}$ tais que $f=x_{1} f_{1}+x_{2} f_{2}+\ldots+x_{n} f_{n}$ onde $x_{1}, x_{2}, \ldots, x_{n}$ são funções coordenadas em $\mathbb{R}^{n}$.

A seguinte proposição determina condições equivalente à $\mathcal{C}$-equivalencia.

Proposição 1.17. ([18]) Sejam $f, g \in \mathbf{m} \mathcal{O}(n, p)$, as seguintes três condições são equivalentes:

1. $f, g$ são $\mathcal{C}$-equivalentes.

2. $I_{f}=I_{g}$ onde $I_{f}=\left\langle f_{1}, f_{2}, \ldots, f_{p}\right\rangle$. 
3. Existe uma matriz invertível $\left(u_{i j}\right)_{p \times p}$ com entradas em $\mathcal{O}_{n}$ onde $f_{i}=\sum u_{i j} g_{j}$ para $1 \leq i \leq p$.

Temos também uma proposição para a $\mathcal{K}$ - equivalência que é análoga à proposição anterior:

Proposição 1.18. As seguintes três condições de germes $f, g \in \mathbf{m} \mathcal{O}(n, p)$ são equivalentes:

1. $f, g$ são $\mathcal{K}$-equivalentes.

2. $I_{f}=I_{g \circ h}$ para algum $h \in \mathcal{R}_{n}$ e onde $I_{f}=\left\langle f_{1}, f_{2}, \ldots, f_{p}\right\rangle$.

3. Existem uma matriz invertível $U=\left(u_{i j}\right)_{p \times p}$ com entradas em $\mathcal{O}_{n}$ e $h \in \mathcal{R}_{n}$ tais que $h^{*}(g)=U \cdot(f) .^{1}$

\subsection{Germes Finitamente Determinados}

Definição 1.19. Seja $G$ um Grupo de Mather ${ }^{2}$, dizemos que $f \in \mathcal{O}_{n}$ é $k$ G-determinado se para todo $g \in \mathcal{O}_{n}$ tal que $j^{k} f(0)=j^{k} g(0), f$ é G-equivalente a $g$.

Dizemos que $f$ é finitamente determinado se $f$ é $k$-determinado para algum $k \in \mathbb{N}$.

O critério infinitesimal de determinação finita dá condições suficientes em termos do espaço tangente para que um germe seja finitamente determinado.

Ele se fundamenta no seguinte teorema.

Teorema 1.20. ( Trivialidade Local, [30]) Seja $F \in \mathbb{C}\left\{x_{1}, \cdots, x_{n}, t\right\}, b \geq 0$ e c $\geq 0$ inteiros.

1. As seguintes afirmações são equivalentes:

(a) $\frac{\partial F}{\partial t} \in\left\langle x_{1}, \cdots, x_{n}\right\rangle^{b} \cdot\left\langle\frac{\partial F}{\partial x_{1}}, \cdots, \frac{\partial F}{\partial x_{n}}\right\rangle+\left\langle x_{1}, \cdots, x_{n}\right\rangle^{c}\langle F\rangle$.

(b) Existem $\phi=\left(\phi_{1}, \cdots, \phi_{n}\right) \in \mathbb{C}\left\{x_{1}, \cdots, x_{n}, t\right\}^{n}, u \in \mathbb{C}\left\{x_{1}, \cdots, x_{n}, t\right\}$ satisfazendo.

(i) $u\left(x_{1}, \cdots, x_{n}, 0\right)=1$,

(ii) $u\left(x_{1}, \cdots, x_{n}, t\right)-1 \in\left(x_{1}, \cdots, x_{n}\right)^{c} \mathbb{C}\left\{x_{1}, \cdots, x_{n}, t\right\}$,

(iii) $\phi_{i}\left(x_{1}, \cdots, x_{n}, 0\right)=x_{i}, \forall i=1, \cdots, n$,

(iv) $\phi_{i}\left(x_{1}, \cdots, x_{n}, t\right)-x_{i} \in\left(x_{1}, \cdots, x_{n}\right)^{b} \mathbb{C}\left\{x_{1}, \cdots, x_{n}, t\right\}, \forall i=1, \cdots, n$,

(v) $u\left(x_{1}, \cdots, x_{n}, t\right) \cdot F\left(\phi\left(x_{1}, \cdots, x_{n}, t\right), t\right)=F\left(x_{1}, \cdots, x_{n}, 0\right)$.

2. A condição: $\frac{\partial F}{\partial t} \in\left\langle x_{1}, \cdots, x_{n}\right\rangle^{b}\left\langle\frac{\partial F}{\partial x_{1}}, \cdots, \frac{\partial F}{\partial x_{n}}\right\rangle$ é equivalente a (1.b) com $u=1$.

${ }^{1} h^{*}(g)=g \circ h$.

${ }^{2}$ os grupos $\mathcal{R}_{n}, \mathcal{C}, \mathcal{K}$ são grupos de Mather 
Demonstração: Notação $x=\left(x_{1}, \cdots, x_{n}\right)$.

$(a) \Longrightarrow(b)$ Temos pela hipótese que $\frac{\partial F}{\partial t} \in\langle x\rangle^{b} \cdot\left\langle\frac{\partial F}{\partial x_{1}}, \cdots, \frac{\partial F}{\partial x_{n}}\right\rangle+\langle x\rangle^{c}\langle F\rangle$ portanto existem $Y_{1}, \cdots, Y_{n} \in(x)^{b} . \mathbb{C}\{x, t\}$ e $Z \in(x)^{c} . \mathbb{C}\{x, t\}$ tais que:

$$
\frac{\partial F}{\partial t}=-\sum_{i=1}^{n} \frac{\partial F}{\partial x_{i}} . Y_{i}-Z . F
$$

Seja $Y=\left(Y_{1}, \cdots, Y_{n}\right)$ e seja a seguinte equação diferencial ordinária.

$$
\frac{\partial \phi}{\partial t}(x, t)=Y(\phi(x, t), t), \text { com condição inicial } \phi(x, 0)=x .
$$

A teoria de equações diferenciais ordinárias nos garante a existência e unicidade da solução de (1.2). Seja $\phi=\left(\phi_{1}, \cdots, \phi_{n}\right)$ a solução para $t$ perto de 0 .

Afirmamos que $\phi_{i}$ satisfaz (iv), de fato suponha que $b \geq 1$ então $Y(0, t)=0$ próximo de $t$, pois $Y \in\langle x\rangle^{b} \mathbb{C}\{x, t\}^{n}$. Assim $\phi=0$ é uma solução da equação diferencial ordinária.

$$
\frac{\partial \phi}{\partial t}(0, t)=Y(\phi(0, t), t), \text { com condição inicial } \phi(0,0)=0 .
$$

Pela unicidade da solução, $\phi(0, t)=0$ o que significa que $\phi_{i} \in(x) . \mathbb{C}\{x, t\}$ e como $Y \in\langle x\rangle^{b} \mathbb{C}\{x, t\}^{n}$ segue-se que

$$
\frac{\partial \phi_{i}}{\partial t}=Y_{i}(\phi(x, t), t) \in(x)^{b} . \mathbb{C}\{x, t\}
$$

e assim $\phi_{i}-x_{i} \in(x)^{b} . \mathbb{C}\{x, t\}$, o que demonstra a afirmação.

Agora seja $\psi(x, t)=(\phi(x, t), t)$, diferenciando $(\mathrm{v})$ com respeito a $\mathrm{t}$, temos:

$$
\frac{\partial}{\partial t}(u \cdot(F \circ \psi)(x, t))=0 .
$$

A condição $(v)$ também vale para $t=0$.

Consideremos a seguinte equação diferenciável ordinária.

$$
\frac{\partial}{\partial t}(x, t)=u(x, t) \cdot(Z \circ \psi)(x, t) \text { com condição inicial } u(x, 0)=1,
$$

e seja $u$ a única solução de $(1.3), Z \in\langle x\rangle^{c}$ implica que $\frac{\partial u}{\partial t} \in\langle x\rangle^{c}$ e assim $u-1 \in$ $\langle x\rangle^{c}$. Usando as equações $(1.1),(1.3)$ e usando a regra da cadeia obtemos. 


$$
\begin{aligned}
\frac{\partial}{\partial t}(u \cdot(F \circ \psi)) & =\frac{\partial u}{\partial t} \cdot(F \circ \psi)+u \frac{\partial(F \circ \psi)}{\partial t} \\
& =u \cdot(Z \circ \psi)(F \circ \psi)+u\left(\sum_{n}^{i=1} \frac{\partial F}{\partial x_{i}} \circ \psi \cdot \frac{\partial \phi_{i}}{\partial t}+\frac{\partial F}{\partial t} \circ \psi\right) \\
& =u \cdot\left((Z \cdot F) \circ \psi+\sum_{n}^{i=1} \frac{\partial F}{\partial x_{i}} \circ \psi \cdot \frac{\partial \phi_{i}}{\partial t}-\sum_{i=1}^{n}\left(\frac{\partial F}{\partial x_{i}} \cdot Y_{i}\right) \circ \psi-(Z . F) \circ \psi\right) \\
& =0,
\end{aligned}
$$

o que é equivalente a $(v)$.

$(b) \Longrightarrow(a)$ Temos que $\psi(x, t)=(\phi(x, t), t)$ define um isomorfismo $\psi:\left(\mathbb{C}^{n} \times\right.$ $\mathbb{C},(0,0)) \rightarrow(\mathbb{C}, 0)$. Defina a inversa como, $\chi=\psi^{-1}$.

Derivando $(v)$ com respeito a $t$ temos:

$$
\begin{aligned}
& u(x, t) \cdot F(\psi(x, t), t)=F(x, 0) \\
& \frac{\partial}{\partial t}(u(x, t) \cdot F(\psi(x, t), t))=0 \\
& \frac{\partial u}{\partial t}(x, t) \cdot F(\psi(x, t))+u\left(\sum_{i=1}^{n} \frac{\partial F}{\partial x_{i}}(\psi(x, t)) \cdot \frac{\phi_{i}}{\partial t}(x, t)+\frac{\partial F}{\partial t}(\psi)\right)=0 .
\end{aligned}
$$

Compondo por $\chi$ e multiplicando por $u$ à esquerda temos:

$$
-\frac{\partial F}{\partial t}=u^{-1}\left(\frac{\partial u}{\partial t} \circ \chi\right) \cdot F+\sum_{i=1}^{n} \frac{\partial \phi_{i}}{\partial t} \circ \chi \cdot \frac{\partial F}{\partial x_{i}} .
$$

Seja $b$ um inteiro positivo, como $\phi_{i}(x, t)-x_{i} \in\langle x\rangle^{b} \mathbb{C}\{x, t\}$ então $\phi(0, t)=0$ e assim $\chi(x, t) \in(\langle x\rangle \mathbb{C}\{x, t\})^{n+1}$. De $(i i)$ temos $u(x, t)-1 \in\langle x\rangle^{c} \mathbb{C}\{x, t\}$, logo, derivando temos que:

$$
\begin{gathered}
\frac{\partial u(x, t)}{\partial t} \in\langle x\rangle^{c} \mathbb{C}\{x, t\} \\
\frac{\partial u(\chi(x, t))}{\partial t} \in\langle x\rangle^{c} \mathbb{C}\{x, t\} .
\end{gathered}
$$

Temos também pela afirmação $(i v)$ que $\phi_{i}-x_{i} \in\langle x\rangle^{b} \mathbb{C}\{x, t\}$ assim a derivada $\frac{\partial \phi_{i}(x, t)}{\partial t} \in\langle x\rangle^{b} \mathbb{C}\{x, t\}$. Então $\frac{\partial \phi_{i}(\chi(x, t))}{\partial t} \in\langle x\rangle^{b} \mathbb{C}\{x, t\}$.

$\mathrm{O}$ resultado segue de (1.5)

A prova da parte (2) é um caso particular de (1) se $Z=0$ então $u=1$ é solução única de (1.3)

O seguinte resultado resulta do Teorema anterior.

Corolário 1.21. Seja $f \in \mathbf{m} \subset \mathbb{C}\{x\}$. 
1. se $\mathbf{m}^{k+1} \subset \mathbf{m}^{2} \cdot\left\langle\frac{\partial f}{\partial x_{1}}, \cdots \frac{\partial f}{\partial x_{n}}\right\rangle$ então $f$ é $k-\mathcal{R}_{n}$ determinado.

2. Se $\mathbf{m}^{k+1} \subset \mathbf{m}^{2} \cdot\left\langle\frac{\partial f}{\partial x_{1}}, \cdots \frac{\partial f}{\partial x_{n}}\right\rangle+\mathbf{m}\langle f\rangle$ então $f$ é $k-\mathcal{K}$ determinado.

\subsection{Deformações de Singularidades}

Na presente seção estudaremos algumas definições clássicas da teoria de deformações de germes de espaços complexos. Definiremos também deformação semiuniversal de um germe de espaços complexos que é uma deformação que contém informação de qualquer deformação destes germes. Um fato fundamental na teoria de deformações é que toda singularidade isolada tem uma deformação versal. As referências para esta seção são: Jan Stevens [31], Michael Artin [3] e Martin Greuel [21].

Sejam $(X, 0)$ e $(S, 0)$ variedades análiticas, $\pi:(X, 0) \rightarrow(S, 0)$ um germe de aplicação analítica, e suponha que a fibra $\left(X_{0}, 0\right)=\left(\pi^{-1}(0), 0\right) \subset\left(\mathbb{C}^{n}, 0\right)$ é dada. Então $\pi$ pode ser considerado como a composição de um mergulho $(X, 0) \subset\left(S \times \mathbb{C}^{n}, 0\right)$ e a projeção de $\left(S \times \mathbb{C}^{n}, 0\right)$ no primeiro fator (ver [21] ).

Seja $\left(F_{1}, F_{2}, \ldots, F_{k}\right)$ gerando o ideal $I$ de $(X, 0)$ em $P=\mathcal{O}_{S, 0} \otimes \mathbb{C}\left\{z_{1}, \ldots, z_{n}\right\}$ onde $\mathcal{O}_{S, 0}$ é o anel local de $S$. As funções $f_{i}(z)=F_{i}(0, z)$ geram o ideal $I_{0}$ de $\left(X_{0}, 0\right)$ em $P_{0}=\mathbb{C}\left\{z_{1} \ldots, z_{n}\right\}$.

Definição 1.22. A aplicação $\pi:(X, 0) \rightarrow(S, 0)$ é plana em 0 se cada relação $\sum f_{i} r_{i}=0 \mathrm{em}$ $f_{i}$ se levanta a uma relação $\sum F_{i} R_{i}=0 \in P$ entre os $F_{i}$. A aplicação $\pi: X \rightarrow S$ entre espaços complexos é plana, se é plana em cada um dos pontos de $X$.

Definição 1.23. Seja $A$ um anel. Um A-módulo $M$ é chamado plano se para qualquer seqüência exata de A módulos

$$
0 \longrightarrow N^{\prime} \longrightarrow N \longrightarrow N^{\prime \prime} \longrightarrow 0
$$

a seguinte seqüência

$$
0 \longrightarrow M \otimes_{A} N^{\prime} \longrightarrow M \otimes_{A} N \longrightarrow M \otimes_{A} N^{\prime \prime} \longrightarrow 0
$$

é também exata. 
Proposição 1.24. (Jan Stevens, [31]) Uma aplicação $\pi:(X, 0) \rightarrow(S, 0)$ é plana em 0 , se somente se $\mathcal{O}_{X, 0}$ é um $\mathcal{O}_{S, 0}$ módulo plano.

Definição 1.25. Uma deformação de um germe $\left(X_{0}, 0\right)$ é uma aplicação plana $\pi:(X, 0) \rightarrow$ $(S, 0)$ tal que $\left(X_{0}, 0\right)$ é isomorfa à fibra $\left(\pi^{-1}(0), 0\right)$ sob um isomorfismo dado $i:\left(X_{0}, 0\right) \rightarrow$ $\left(\pi^{-1}(0), 0\right)$.

Definição 1.26. Um morfismo entre duas deformações $\pi:(X, 0) \rightarrow(S, 0)$ e $\pi^{\prime}:\left(X^{\prime}, 0\right) \rightarrow$ $(S, 0)$ de $\left(X_{0}, 0\right)$ sob a mesma base $(S, 0)$ é um morfismo $f:(X, 0) \rightarrow\left(X^{\prime}, 0\right)$ sobre $(S, 0)^{3}$, compatível com as aplicações $i:\left(X_{0}, 0\right) \rightarrow(X, 0) e i^{\prime}:\left(X_{0}, 0\right) \rightarrow\left(X^{\prime}, 0\right){ }^{4}$

Dados os morfismos entre espaços complexos $\phi: X \rightarrow S$ e $\varphi: T \rightarrow S$, definimos a fibra produto (ou pullback) de $X$ e $T$ sobre $S$ como sendo a tripla $\left(X \times_{S} T, \pi_{X}, \pi_{T}\right)$ tal que o seguinte diagrama comuta.

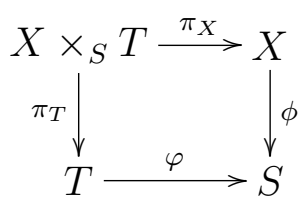

e satisfaz a propriedade universal, ou seja: dados $Z$ um espaço complexo, $f: Z \rightarrow X$ e $g: Z \rightarrow T$ tais que $\phi \circ f=\varphi \circ g$ então existe um único morfismo $h: Z \rightarrow X \times{ }_{S} T$ tal que o seguinte diagrama comuta:

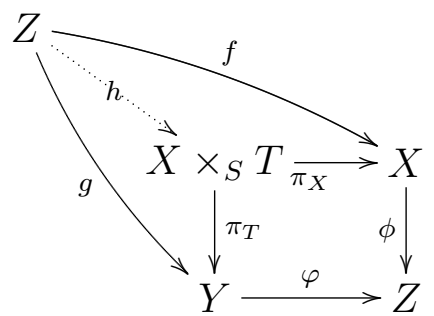

A existência deste espaço é dada considerando $D=X \times{ }_{S} T=\{(x, t) \in X \times$ $T / \varphi(t)=\phi(x)\}$ e as aplicacões $\pi_{X}$ e $\pi_{T}$ são dadas pelas projeções.

Introduziremos agora a definição de deformação induzida, que surge de maneira natural considerando deformações sobre bases diferentes.

Seja $\left(X_{0}, 0\right) \longrightarrow(X, 0) \stackrel{\pi}{\longrightarrow}(S, 0)$ uma deformação e $\varphi:(T, t) \rightarrow(S, 0)$ um morfismo de germes, então temos o seguinte diagrama comutativo

\footnotetext{
${ }^{3} \pi^{\prime} \circ f=\pi$

${ }^{4} f \circ i=i^{\prime}$
} 


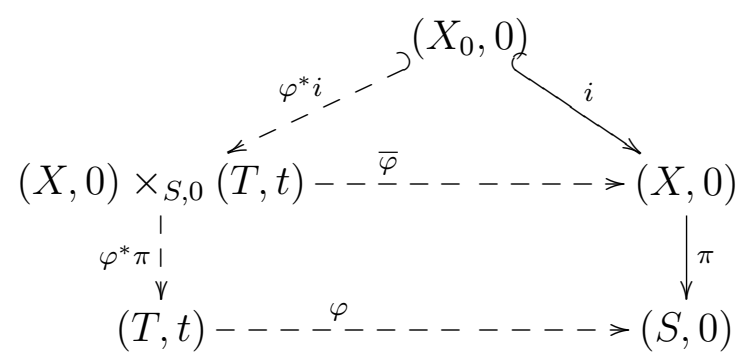

onde $\varphi^{*} \pi$, respectivamente $\bar{\varphi}$ são induzidas pela segunda e primeira projeção e:

$$
\varphi^{*} i=\left(\left.\bar{\varphi}\right|_{\left(\varphi^{*} \pi\right)^{-1}}\right)^{-1} \circ i
$$

Definição 1.27. Seja $\pi:(X, 0) \rightarrow(S, 0)$ uma deformação de $\left(X_{0}, 0\right)$ e seja $\varphi:(T, 0) \rightarrow(S, 0)$ uma aplicação holomorfa. A deformação induzida é a aplicação $\varphi^{*}(\pi):\left(X \times_{S} T, 0\right) \rightarrow(T, 0)$.

Definição 1.28. Uma deformação $\pi:(X, 0) \rightarrow(S, 0)$ de $\left(X_{0}, 0\right)$ é chamada universal ou semiuniversal se cada deformação $\rho:(Y, 0) \rightarrow(T, 0)$ de $\left(X_{0}, 0\right)$ é isomorfa à deformação $\varphi^{*}(\pi)$, para alguma aplicação $\varphi:(T, 0) \rightarrow(S, 0)$; a aplicação $\varphi$ não precisa ser única, mas a derivada tem que ser única.

Teorema 1.29. (Greuel-Lossen-Shustin, [21]) Seja $\left(X_{0}, 0\right)$ um germe de uma singularidade isolada. Então existe uma deformação semiuniversal de $\left(X_{0}, 0\right)$.

Uma deformação de primeira ordem de $X \subset \mathbb{C}^{n}$ é uma deformação sob $\mathbb{C}\{\varepsilon\}$, onde $\varepsilon$ é definido como uma variável com a propriedade que $\varepsilon^{2}=0$. Denotamos por $\operatorname{Der}_{\mathbb{C}}\left(\mathcal{O}_{\mathbb{C}^{m}}\right)$ o módulo dos germes de campos vetores analíticos na origem.

Definição 1.30. Seja $\left(X_{0}, 0\right) \subset\left(\mathbb{C}^{m}, 0\right)$ o germe de uma variedade analítica complexa, com singularidade isolada na origem e $\mathcal{O}_{X_{0}, 0}=\mathbb{C}\{x\} /$ I seu anel local.

- O espaço $N_{\left(X_{0}, 0\right)}$ das deformações mergulhadas de $1^{a}$ ordem de $\left(X_{0}, 0\right)$, é dado por

$$
N_{X_{0}, 0}=\operatorname{Hom}_{\mathcal{O}_{X_{0}}, 0}\left(I / I^{2}, \mathcal{O}_{X_{0}, 0}\right) .
$$

Alguns autores também se referem a $N_{\left(X_{0}, 0\right)}$ como espaço normal de $\left(X_{0}, 0\right)$.

- O espaço vetorial das deformações de primeira ordem de $\left(X_{0}, 0\right)$, que indicamos por $T^{1}\left(X_{0}, 0\right)$,é definido pela seguinte seqüência exata:

$$
\begin{gathered}
0 \longrightarrow \operatorname{Der}_{\mathbb{C}}\left(\mathcal{O}_{\mathbb{C}^{m}}\right) \longrightarrow \mathcal{O}_{X_{0}, 0} \otimes \operatorname{Der}_{\mathbb{C}}\left(\mathcal{O}_{\mathbb{C}^{m}}\right) \longrightarrow N_{X_{0}, 0} \longrightarrow T_{\left(X_{0}, 0\right)}^{1} \longrightarrow 0 \\
\frac{\partial}{\partial x_{i}} \vdash----->\left(f_{j} \vdash-->\frac{\partial f_{j}}{\partial x_{i}}\right) .
\end{gathered}
$$




\subsection{A Profundidade e a Codimensão de um Ideal}

Definição 1.31. Sejam $R$ um anel e $M$ um $R$-módulo. Uma seqüência $\left(a_{1}, \ldots, a_{n}\right)$ de elememtos de $R$ é regular em relação à $M$ ou uma $M$-seqüência se:

1. $a_{i}$ não é um divisor de zero em $M /\left\langle a_{1}, \ldots, a_{i-1}\right\rangle$ M para $i=1, \ldots, n$;

2. $M \neq\left\langle a_{1}, \ldots, a_{n}\right\rangle M$.

Se $R$ é um anel Noetheriano e $\mathrm{x}=\left(x_{1}, \ldots, x_{n}\right)$ é uma seqüência, então podemos estender a seqüência ascendente $\left(x_{1}\right) \subset\left(x_{1}, x_{2}\right) \subset \ldots \subset\left(x_{1}, \ldots, x_{n}\right)$ para uma seqüência maximal.

Definição 1.32. Dado um anel $R$ Noetheriano, $M$ um $R$-módulo e I um ideal próprio $I \subset R$, então a profundidade (ou depth) de I em $M$, denotado por depth $(I, M)$, é definida como o comprimento máximo de qualquer $M$-seqüência contida no ideal $I$.

Quando $M=R$, escrevemos simplesmente $\operatorname{depth}(I)$. Se $M=I M$, então $\operatorname{depth}(I, M)$ é por convensão, infinito. Quando $(R, \mathbf{m})$ é um anel local, então o $\operatorname{depth}(\mathbf{m}, M)$ é simplesmente denotado por depth $(M)$.

Definição 1.33. A dimensão de Krull (ou dimensão) de um anel $R$ é o supremo dos comprimentos das cadeias:

$$
P_{0} \subsetneq P_{1} \subsetneq \ldots \subsetneq P_{r-1} \subsetneq P_{r}
$$

onde cada $P_{i}$ é ideal primo $P_{i} \subsetneq R$ para todo $i$.

Se $I$ é um ideal em $R$, definimos a dimensão de $I$ como sendo a dimensão do anel $R / I$.

Definição 1.34. Seja $R$ um anel e $P$ um ideal primo de $R$. Definimos a altura de $P$, denotada por $h t(P)$, pelo supremo dos comprimentos das cadeias de ideais

$$
P_{0} \subsetneq P_{1} \subsetneq \ldots \subsetneq P_{r}
$$

tais que $P_{i}$ é ideal primo, para todo $i$ e $P_{r}=P$

Se I é um ideal arbitrário de $R$, definimos a altura (ou codimensão) de I como

$$
h t(I)=\inf \{h t(P) / P \text { é ideal primo de Re } P \supset I\}
$$

Teorema 1.35. ([14]) Dado um ideal I de um anel Noetheriano $R$, então

$$
\operatorname{depth}(I, R) \leq \operatorname{codim}(I) .
$$


Definição 1.36. ([14]) Dizemos que um anel Noetheriano $R$ é um anel Cohen-Macaulay se $\operatorname{depth}(\mathbf{m})=\operatorname{codim}(\mathbf{m})$ para todo ideal maximal $\mathbf{m}$ de $R$.

Exemplo 1.37. O anel $\mathbb{C}\left\{x_{1}, \ldots, x_{m}\right\}$ é Cohen-Macaulay.cz $z v c x$

Definição 1.38. (Anéis Determinantais) Um anel $R$ é determinantal se pode ser escrito da forma $R=\frac{S}{I}$ onde $S$ é um anel Cohen-Macaulay e $I$ é o ideal de $R$ gerado pelos menores de tamanho $r \times r$ de uma matriz $M$ de tamanho $p \times q$ com entradas em $R$, para $p, q, r$ tais que a codimensão de I em $S$ é $(p-r+1)(q-r+1)$.

Observação 1.39. ([21]) Anéis Determinantais são Cohen-Macaulay.

Definição 1.40. Um anel local Noetheriano $(R, \mathbf{m}, \mathbb{K})$ é regular se $\operatorname{dim} A=\operatorname{dim}_{\mathbb{K}} \frac{\mathbf{m}}{\mathbf{m}^{2}}$.

Dizemos que um anel $R$ é uma intersecção completa se existe um anel regular $S$ e uma seqüência regular $x_{1}, \ldots, x_{m} \in S$ tais que $R \cong S /\left(x_{1}, \ldots, x_{m}\right)$.

Interseções completas são determinantais e portanto Cohen-Macaulay.

Definição 1.41. (Joseph Rotman, [28]) Uma resolução projetiva de um $R$ - módulo $M$ é um complexo

$$
\mathcal{F}: \ldots \longrightarrow F_{n} \stackrel{\phi_{n}}{\longrightarrow} F_{n-1} \stackrel{\phi_{n-1}}{\longrightarrow} \cdots \longrightarrow F_{1} \stackrel{\phi_{1}}{\longrightarrow} M \longrightarrow 0
$$

de $R$ - módulos projetivos tais que coker $\phi=M$ e $\mathcal{F}$ tem homologia nula ${ }^{5}$. Se cada módulo $F_{i} e ́$ livre, então $\mathcal{F}$ é chamada resolução projetiva livre.

Dizemos que $\mathcal{F}$ é uma resolução projetiva finita de comprimento $n$, se $F_{n+1}=0$ e $F_{i} \neq 0$ para $0 \leq i \leq n$. A dimensão projetiva de $M$, denotada por $p d(M)$, é o número mínimo dos comprimentos das resoluções projetivas de $M$.

Definição 1.42. Seja $R$ um anel Noetheriano local e $M$ um $R$-módulo não nulo. Dizemos que $M$ é perfeito se $p d(M)=\operatorname{depth}(M)$. Se I é um ideal de $R$, então I é perfeito se depth $(I)=$ $\operatorname{pd}(R / I)$.

Teorema 1.43. (Fórmula de Auslander-Buchsbaum, [21]) Seja R um anel local e M um Rmódulo finitamente gerado com dimensão projetiva finita. Então

$$
p d(M)+\operatorname{depth}(M)=\operatorname{depth}(A)
$$

\footnotetext{
${ }^{5} \mathrm{~A}$ homologia do complexo em $F_{n}$ é o módulo $\operatorname{ker} \phi_{n} / \operatorname{Im} \phi_{n+1}$
} 


\subsection{Teorema de Hilbert-Burch}

O Teorema de Hilbert-Burch fornece uma descrição para ideais perfeitos usando uma matriz $M,(n+1) \times n$, e é muito usado no estudo de anéis Cohen-Macaulay de codimensão 2 , que serão estudados na seguinte seção.

Teorema 1.44. (Caracterização de Resoluções Livres [14], página 496) Seja $R$ um anel Noetheriano. Então o complexo de módulos livres finitamente gerados:

$$
\mathcal{F}: 0 \longrightarrow F_{n} \stackrel{\phi_{n}}{\longrightarrow} F_{n-1} \stackrel{\phi_{n-1}}{\longrightarrow} \cdots \longrightarrow F_{1} \stackrel{\phi_{1}}{\longrightarrow} F_{0}
$$

é um complexo exato se somente se as seguintes condições se cumprem:

1. $\operatorname{rank}\left(F_{k}\right)=\operatorname{rank}\left(\phi_{k}\right)+\operatorname{rank}\left(\phi_{k+1}\right), \forall k=1, \cdots, n-1 \operatorname{erank}\left(F_{n}\right)=\operatorname{rank}\left(\phi_{n}\right)$,

2. $\operatorname{depth}\left(I\left(\phi_{k}\right)\right) \geq k, \forall k=1, \cdots, n$.

onde $I\left(\phi_{k}\right)$ é o ideal gerado pelos menores de tamanho $r \times r, r=\operatorname{rank} \phi_{k}$.

Observação 1.45. O complexo exato acima descreve uma resolução livre de $\frac{F_{0}}{\operatorname{Im}\left(\phi_{1}\right)}$

$$
\mathcal{F}: 0 \longrightarrow F_{n} \stackrel{\phi_{n}}{\longrightarrow} F_{n-1} \stackrel{\phi_{n-1}}{\longrightarrow} \cdots \longrightarrow F_{1} \stackrel{\phi_{1}}{\longrightarrow} \operatorname{Im}\left(\phi_{1}\right) \stackrel{\psi}{\longrightarrow} \frac{F_{0}}{\operatorname{Im}\left(\phi_{1}\right)}
$$

Corolário 1.46. Seja $R$ um anel Noetheriano. Qualquer Ideal I não trivial de R que tem uma resolução livre contém um elemento a não divisor de 0.

Teorema 1.47. (Teorema de Hilbert-Burch [14], página 510) Seja $R$ um anel Noetheriano e I um ideal não trivial próprio de $R$.

1. Se o complexo

$$
\mathcal{F}: 0 \longrightarrow F_{2} \stackrel{\phi_{2}}{\longrightarrow} F_{1} \stackrel{\phi_{1}}{\longrightarrow} R \longrightarrow \frac{R}{I} \longrightarrow 0
$$

é exato e $F_{1} \cong R^{n+1}$, então $F_{2} \cong R^{n}$ e existe " a" não divisor de zero tal que $I=a I_{n}\left(\phi_{2}\right)$. De fato a $i$ - ésima entrada da matriz de $\phi_{1} e ́(-1)^{i}$ a vezes o menor obtido de $\phi_{2}$ eliminando a $i$-ésima linha. O ideal $I_{n}\left(\phi_{2}\right)$ tem profundidade igual a 2.

2. Dada uma matriz $\phi_{2}$ de tamanho $n \times(n+1)$ tal que depth $I\left(\phi_{2}\right) \geq 2$, e " a" não divisor de zero, a aplicação $\phi_{1}$ obtida como na parte 1, faz $\mathcal{F}$ uma resolução livre de $\frac{R}{I}$, com $I=a I_{n}\left(\phi_{2}\right)$. 


\section{Demonstração:}

1. Temos que o complexo é exato:

$$
\mathcal{F}: 0 \longrightarrow F_{2} \stackrel{\phi_{2}}{\longrightarrow} F_{1} \stackrel{\phi_{1}}{\longrightarrow} R \stackrel{\phi}{\longrightarrow} \frac{R}{I} \longrightarrow 0
$$

Então $\operatorname{Im}\left(\phi_{1}\right)=\operatorname{ker}(\phi)=I, \phi_{1} ¥ 0$ portanto existe ao menos um elemento da matriz $\phi_{1}$ não nulo, assim temos que $\operatorname{rank}\left(\phi_{1}\right)=1$.

Pelo Teorema (1.44) temos que:

$\operatorname{rank}\left(F_{1}\right)=\operatorname{rank}\left(\phi_{1}\right)+\operatorname{rank}\left(\phi_{2}\right)$ então $n+1=1+\operatorname{rank}\left(\phi_{2}\right)$, ademais $\operatorname{rank}\left(F_{2}\right)=$ $\operatorname{rank}\left(\phi_{2}\right)=n$ o que mostra a primeira parte de (1).

Por outro lado $\operatorname{depth}\left(I\left(\phi_{2}\right)\right)=\operatorname{depth}\left(I_{n}\left(\phi_{2}\right)\right) \geq 2$, usando Teorema (1.35) e a Observação 1.38) se tem:

$$
\operatorname{depth}\left(I_{n}\left(\phi_{2}\right)\right) \leq \operatorname{codim}\left(I_{n}\left(\phi_{2}\right)\right)=((n+1)-n+1)(n-n+1)=2
$$

portanto temos que $\operatorname{depth}\left(I_{n}\left(\phi_{2}\right)\right)=2$

Defina o seguinte homomorfismo de $R$ módulos.

$$
\begin{aligned}
\Delta: F_{1} & \longrightarrow R \\
e_{i} & \longmapsto \Delta\left(e_{i}\right)=\Delta_{i},
\end{aligned}
$$

onde $\Delta_{i}$ é o menor maximal da matriz de $\phi_{2}$ deixando a i-ésima linha. Assim:

$$
0 \longrightarrow F_{2} \stackrel{\phi_{2}}{\longrightarrow} F_{1} \stackrel{\Delta}{\longrightarrow} R \longrightarrow \frac{R}{I} \longrightarrow 0
$$

é um complexo. De fato, $\Delta \circ \phi_{2}=\sum_{j=0}^{n+1} \Delta_{j} \phi_{2}^{j i}=0$, pois é o determinante da matriz $\phi_{2}$ aumentando a i-ésima coluna da mesma $\phi_{2}$. Portanto temos dois complexos:

$$
\begin{gathered}
\mathcal{F}: 0 \longrightarrow F_{2} \stackrel{\phi_{2}}{\longrightarrow} F_{1} \stackrel{\phi_{1}}{\longrightarrow} R \longrightarrow \frac{R}{I} \longrightarrow 0, \\
\mathcal{F}^{\prime}: 0 \longrightarrow F_{2} \stackrel{\phi_{2}}{\longrightarrow} F_{1} \stackrel{\Delta}{\longrightarrow} R \longrightarrow \frac{R}{I} \longrightarrow 0 .
\end{gathered}
$$


Aplicando o funtor contra-variante $\operatorname{Hom}_{R}(-, R)$ aos complexos temos:

$$
\begin{aligned}
& \mathcal{F}^{*}: 0 \longrightarrow R^{*} \stackrel{\phi_{1}^{*}}{\longrightarrow} F_{1}^{*} \stackrel{\phi_{2}^{*}}{\longrightarrow} F_{2}^{*}, \\
& \mathcal{F}^{*^{\prime}}: 0 \longrightarrow R^{*} \stackrel{\Delta^{*}}{\longrightarrow} F_{1}^{*} \stackrel{\phi_{2}^{*}}{\longrightarrow} F_{2}^{*} .
\end{aligned}
$$

Provamos que $R^{*} \cong R, F_{1}^{*} \cong F_{1}, F_{2}^{*} \cong F_{2}$ usando este homomorfismo que de fato é um isomorfismo.

$$
\begin{aligned}
\cdot: F_{1}^{*} & \longrightarrow F_{1} \cong R^{n+1} \\
f & \longmapsto\left(f\left(e_{1}\right), \cdots, f\left(e_{n+1}\right)\right),
\end{aligned}
$$

onde $\left(e_{1}, \ldots, e_{n}\right)$ é uma base de $F_{1}$. Também se mostra que as aplicações $\phi_{1}^{*}=\phi_{1}^{T}$, $\phi_{2}^{*}=\phi_{2}^{T}$ e $\Delta^{*}=\Delta^{T}$.

Portanto temos os seguintes complexos:

$$
\begin{gathered}
\mathcal{F}^{*}: 0 \longrightarrow R \stackrel{\phi_{1}^{T}}{\longrightarrow} F_{1} \stackrel{\phi_{2}^{T}}{\longrightarrow} F_{2} \\
\mathcal{F}^{*^{\prime}}: 0 \longrightarrow R \stackrel{\Delta^{T}}{\longrightarrow} F_{1} \stackrel{\phi_{2}^{T}}{\longrightarrow} F_{2} .
\end{gathered}
$$

Sabemos que $\operatorname{rank}\left(\phi_{2}^{T}\right)=\operatorname{rank}\left(\phi_{2}\right)=n$ e $\operatorname{depth}\left(I\left(\phi_{2}^{T}\right)\right)=\operatorname{depth}\left(I\left(\phi_{2}\right)\right) \geq 2 \geq 1$. Como $\operatorname{rank}\left(\phi_{2}\right)=n$ então existe ao menos uma sub-matriz de $\phi_{2}$ com determinante distinto de 0 , portanto ao menos algum $\Delta_{i} \neq 0$, assim $\operatorname{rank}\left(\Delta^{T}\right)=1$, e de fato $I\left(\Delta^{T}\right)=I_{1}\left(\Delta^{T}\right)=I_{t}\left(\phi_{2}\right)$.

Agora $\operatorname{depth}\left(I\left(\Delta^{T}\right)\right)=\operatorname{depth}\left(I\left(\phi_{2}\right)\right) \geq 2$. Como $\operatorname{rank}\left(\Delta^{T}\right)=1=\operatorname{rank}(R)$, $\operatorname{rank}\left(F_{1}\right)=n+1=\operatorname{rank}\left(\phi_{2}^{T}\right)+\operatorname{rank}\left(\Delta^{T}\right)$, então pelo Teorema 1.44 se tem que $\mathcal{F}^{*^{\prime}}$ é um complexo exato. Dada a aplicação $I d: F_{2} \rightarrow F_{2}$, então existem aplicações de cadeias tais que o digrama comuta.

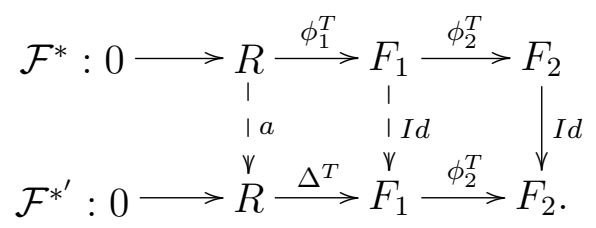

Como as aplicações são únicas salvo homotopia temos que $I d: F_{1} \rightarrow F_{1}$ satisfaz 
o quadrado direito e defina

$$
\begin{aligned}
a: R & \longrightarrow R \\
r & \longmapsto a . r
\end{aligned}
$$

Portanto $\phi_{1}^{T}=\Delta^{T} \circ$ a que é o mesmo que $\phi_{1}\left(e_{i}\right)=\Delta_{i} . a$. Assim $I=\operatorname{Im}\left(\phi_{1}\right)=$ $\left\langle\Delta_{1} . a, \cdots, \Delta_{n+1} . a\right\rangle=a I_{n}\left(\phi_{2}\right)$.

$I=a \cdot I_{n}\left(\phi_{2}\right)$ tem uma resolução livre por hipótese, pelo Corolário 1.46 existe um $r \in I$ que não é divisor de zero. Assim $r \in\langle a\rangle$, ou seja $r=a s$ para algum $s \in R$. Suponha que $a$ é um divisor de zero, então $a u=0$ para algum $u \in R$. Assim, $u r=u a s=0$, fazendo $r$ um divisor de zero, o que é uma contradição.

2. Agora suponha que temos uma matriz $\phi_{2}$ de tamanho $(n+1) \times n$ tal que $\operatorname{depth}\left(\phi_{2}\right) \geq$ 2 e um elemento $a \in R$ que não é divisor de 0 .

Como o depth $\left(I_{n}\left(\phi_{2}\right)\right) \geq 2$ então $\phi_{2} \neq 0$, portanto $\operatorname{rank}\left(\phi_{2}\right) \geq n$ e como $\phi_{2}$ é uma matriz de tamanho $(n+1) \times n$ então $\operatorname{rank}\left(\phi_{2}\right)=n$.

Considerando $\Delta$ como na parte $(1)$, temos que $I_{1}(\Delta)=I_{t}\left(\phi_{2}\right) \neq 0$ e como a não é divisor de zero, $I_{1}(a . \Delta)=a \cdot I_{1}(\Delta) \neq 0$. Assim $\operatorname{rank}(a \Delta) \geq 1$.

Além disso note que $I(a . \Delta)=I_{1}(a . \Delta)=a I_{1}(\Delta)=a I\left(\phi_{2}\right)$, e pela hipótese temos que $\operatorname{depth}(I(a . \Delta)) \geq 2 \geq 1$. Por tanto, pelo Teorema (1.44) temos um complexo exato da forma.

$$
\mathcal{F}: 0 \longrightarrow R^{n} \stackrel{\phi_{2}}{\longrightarrow} R^{n+1} \stackrel{a \Delta}{\longrightarrow} R \longrightarrow \frac{R}{a \Delta} \longrightarrow 0
$$

Usando os isomorfismos $F^{1} \cong R^{n+1}$ e $F^{2} \cong R^{n}$

$$
\mathcal{F}: 0 \longrightarrow F_{2} \stackrel{\phi_{2}}{\longrightarrow} F_{1} \stackrel{a \Delta}{\longrightarrow} R \longrightarrow \frac{R}{a \Delta} \longrightarrow 0 \text {. }
$$




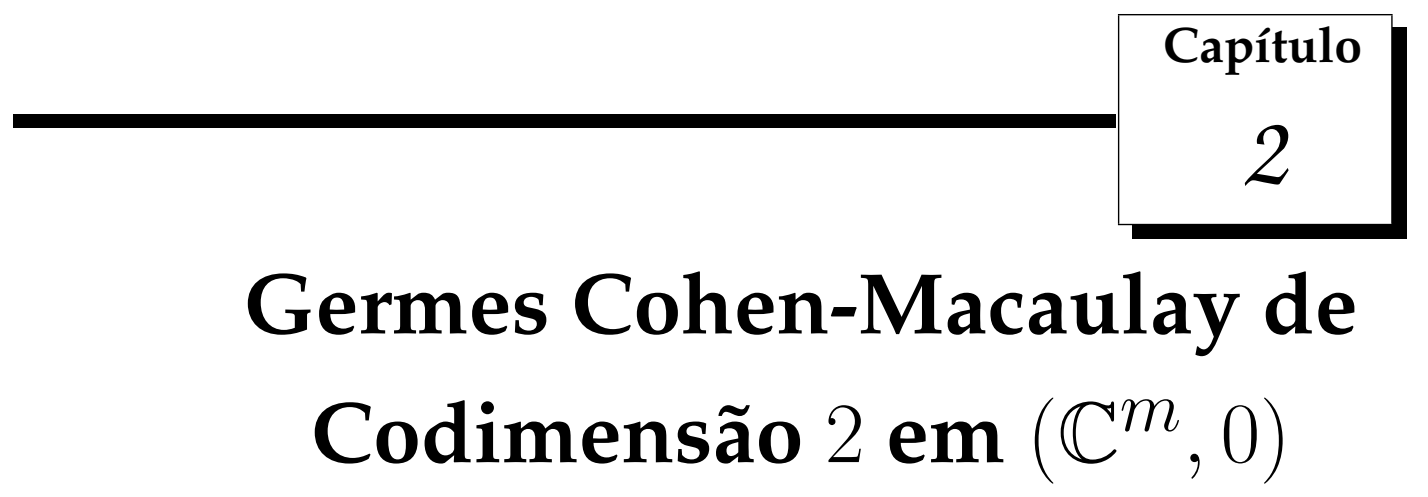

Os objetivos deste capítulo são enunciar uma aplicação do Teorema de HilbertBurch para anéis Cohen- Macaulay de codimensão 2, definir o grupo $\mathcal{G}(n)$ e encontrar critérios de determinação finita de matrizes. Além disso calcularemos os espaço normal, $N_{X}$, e o espaço das deformações de primeira ordem, $T_{X}^{1}$, em função da matriz de representação de $X$, onde $X=V(I)$ é uma variedade Cohen-Macaulay de codimensão dois.

Na seqüência usaremos a notação $\mathcal{P}$ para representar o anel $\mathcal{O}_{m}$ e $\mathbb{K}[[x]]$ para representar o anel das séries formais sobre $\mathbb{K}$.

\subsection{Descrição da Matriz de Representação}

Podemos usar o Teorema de Hilbert-Burch (1.47), para mostrar a seguinte proposição que diz que todo germe Cohen-Macaulay de codimensão 2 pode ser expresso como os menores maximais de uma matriz $M$ de tamanho $(n+1) \times n$ e viceversa. No mesmo contexto, deformações de um germe Cohen-Macaulay de codimensão 2 podem ser representadas por perturbações da matriz $M$, e qualquer perturbação da matriz surge como uma deformação do germe $X$. Tal matriz $M$ será chamada matriz de representação.

\section{Proposição 2.1.}

1. Sejam $M \in \mathcal{M a t}_{(n+1) n}\left(\mathbb{C}\left\{x_{1}, x_{2}, \cdots, x_{m}\right\}\right), e \Delta=\left(\Delta_{1}, \Delta_{2}, \cdots, \Delta_{n+1}\right)$ o ideal gerado por seus menores maximais (o indice de $\Delta_{i}$ indica o indice da linha de $M$ que foi elimi- 
nada para calcular o menor).

Se a $\operatorname{codim}(V(\Delta)) \geq 2$, a seguinte seqüência

$$
0 \longrightarrow \mathcal{P}^{n} \stackrel{M}{\longrightarrow} \mathcal{P}^{n+1} \stackrel{\Delta}{\longrightarrow} \mathcal{P} \longrightarrow \frac{\mathcal{P}}{(\Delta)} \longrightarrow 0
$$

é exata. Então, $\frac{\mathcal{P}}{(\Delta)}$ é Cohen - Macaulay e codim $(V(\Delta))=2$.

2. Se $X=V(I) \subseteq \mathbb{C}^{m}$ é Cohen- Macaulay, tal que codim $(V(I))=2$ então $\mathcal{P} / I$ tem uma resolução livre minimal do tipo.

$$
0 \longrightarrow \mathcal{P}^{n} \stackrel{M}{\longrightarrow} \mathcal{P}^{n+1} \stackrel{I}{\longrightarrow} \mathcal{P} \longrightarrow \frac{\mathcal{P}}{I} \longrightarrow 0
$$

Além disso existe uma unidade $u \in \mathcal{P}$ tal que $I=u . \Delta$, onde $\Delta=\left\langle\Delta_{1}, \ldots, \Delta_{n+1}\right\rangle$.

3. Qualquer perturbação da matriz $M$ dá origem a uma deformação do $X$.

4. Qualquer deformação da variedade X pode ser gerada por uma perturbação da matriz $M$.

Observação 2.2. $X \subseteq \mathbb{C}^{m}$ é chamado Cohen-Macaulay se o anel local , $\frac{\mathcal{P}}{I}$,é Cohen-Macaulay

\section{Demonstração:}

1. Como $\operatorname{codim} V(\Delta) \geq 2$ então $\operatorname{depth}(\Delta) \geq 2$. Pelo Teorema de Hilbert-Burch 1.47 , parte $(2)$, temos que a seqüência

$$
0 \longrightarrow \mathcal{P}^{n} \stackrel{M}{\longrightarrow} \mathcal{P}^{n+1} \stackrel{\Delta}{\longrightarrow} \mathcal{P} \longrightarrow \frac{\mathcal{P}}{(\Delta)} \longrightarrow 0
$$

é exata. Além disso, depth $(\Delta)=2$. Portanto $\mathcal{P} / \Delta$ é Cohen Macaulay.

2. Considere $I=\left\langle f_{1}, \ldots, f_{n+1}\right\rangle$, como a $\operatorname{codim} V(I)=2$ então $\operatorname{depth} P / I=\mathrm{m}-2$, (ver [3]). Usando a Fórmula de Auslander-Buchsbaum (1.43) temos que depthP/I+ $h d_{P} P / I=m$, $\operatorname{logo} h d_{P} P / I=2$, ou seja $P / I$ tem uma resolução projetiva de comprimento dois.

$$
0 \longrightarrow F \stackrel{M}{\longrightarrow} \mathcal{P}^{n+1} \stackrel{I}{\longrightarrow} \mathcal{P} \longrightarrow \frac{\mathcal{P}}{I} \longrightarrow 0
$$

Pelo Teorema de Hilbert- Burch (1.47), temos que $F \cong \mathcal{P}^{n}$. Além disso existe $u \in \mathcal{P}$ tal que $I=u \Delta$, onde $\Delta$ é o ideal gerado pelos menores maximais da matriz $M$. 
3. Seja $X=V(I) \subseteq \mathbb{C}^{m}$ uma variedade Cohen-Macaulay de codimensão 2 então pela parte $(2), \mathcal{P} / I$ admite uma seqüência exata do tipo

$$
(*) \quad 0 \longrightarrow \mathcal{P}^{n} \stackrel{M}{\longrightarrow} \mathcal{P}^{n+1} \stackrel{I}{\longrightarrow} \mathcal{P} \longrightarrow \frac{\mathcal{P}}{\Delta} \longrightarrow 0
$$

onde $I=u . \Delta$, para alguma unidade $u$ de $\mathcal{P}$.

Seja $\widetilde{M}$ uma perturbação de $M$ sobre $\mathcal{P}_{A}=A[x]$, onde $A=\mathbb{C}\{e\} /\left\langle e^{2}\right\rangle$. Indiquemos por $M_{i j}^{\prime}$ e $M_{i j}$ as entradas da matriz $\widetilde{M}$ e $M$ respectivamente tais que temos o seguinte homomorfismo canônico:

$$
\begin{aligned}
\rho: \mathcal{P}_{A} & \longrightarrow \mathcal{P} \\
M_{i j}^{\prime} & \longmapsto M_{i j}
\end{aligned}
$$

Defina-se $\Delta_{i}^{\prime}$ como sendo o menor maximal de $M^{\prime}$ cujo índice indica o índice da linha de $M^{\prime}$ que foi eliminada para calcular o menor, e considere-se $\Delta^{\prime}=$ $\left(\Delta_{1}^{\prime}, \ldots, \Delta_{n+1}^{\prime}\right)$. Como $\operatorname{codim} V(\Delta) \geq 2$ então $\operatorname{codim} V\left(\Delta^{\prime}\right) \geq 2$ e pela parte $(2)$ do Teorema de Hilbert-Burch (1.47), existe uma seqüência exata do tipo:

$$
(* *) \quad 0 \longrightarrow \mathcal{P}_{A}^{n} \stackrel{M^{\prime}}{\longrightarrow} \mathcal{P}_{A}^{n+1} \stackrel{\Delta^{\prime}}{\longrightarrow} \mathcal{P}_{A} \longrightarrow \frac{\mathcal{P}_{A}}{\Delta^{\prime}} \longrightarrow 0
$$

Temos que $(* *) \otimes \mathbb{K}=(*)$, portanto $\frac{\mathcal{P}_{A}}{\Delta^{\prime}}$ é um $A$ módulo plano.

Isto demonstra que qualquer deformação $M^{\prime}$ de $M$ dá origem a uma deformação $X_{A}=V\left(\Delta^{\prime}\right)$ de $X=V(\Delta)$.

4. Seja $X=V(\Delta)$ uma variedade Cohen-Macaulay de codimensão dois então pela parte (2), $\mathcal{P} / I$ admite uma seqüência exata do tipo

$$
(*) \quad 0 \longrightarrow \mathcal{P}^{n} \stackrel{M}{\longrightarrow} \mathcal{P}^{n+1} \stackrel{I}{\longrightarrow} \mathcal{P} \longrightarrow \frac{\mathcal{P}}{\Delta} \longrightarrow 0
$$

onde $I=u . \Delta$, para alguma unidade $u$ de $\mathcal{P}$.

Seja $X_{A}$ uma deformação infinitesimal de $X=V(\Delta)$ então $X_{A}=V\left(I_{A}\right)$ onde $I_{A}=\left(f_{1}, \ldots, f_{n+1}\right)$. Como $\operatorname{codim} V(\Delta) \geq 2$ então $\operatorname{codim} V\left(\Delta^{\prime}\right) \geq 2$ e pela parte $(2)$ do Teorema de Hilbert-Burch (1.47), existe uma seqüência exata do tipo:

$$
\left(* *^{\prime}\right) \quad 0 \longrightarrow \mathcal{P}_{A}^{n} \stackrel{M^{\prime}}{\longrightarrow} \mathcal{P}_{A}^{n+1} \stackrel{\Delta^{\prime}}{\longrightarrow} \mathcal{P}_{A} \longrightarrow \frac{\mathcal{P}_{A}}{I_{A}} \longrightarrow 0 .
$$

Sejam $\Delta_{i}^{\prime}$ os determinantes dos menores maximais de $M^{\prime}$ e considere o ideal $J_{A}=$ 
$\left(\Delta_{1}^{\prime}, \ldots, \Delta_{n+1}\right)$, então temos que a seguinte seqüência é exata.

$$
0 \longrightarrow \mathcal{P}_{A}^{n} \stackrel{M^{\prime}}{\longrightarrow} \mathcal{P}_{A}^{n+1} \stackrel{\Delta^{\prime}}{\longrightarrow} \mathcal{P}_{A} \longrightarrow \frac{\mathcal{P}_{A}}{J_{A}} \longrightarrow 0
$$

considerando os duais de $(* *)$ e $\left(* *^{\prime}\right)$ temos o seguinte diagrama comutativo.

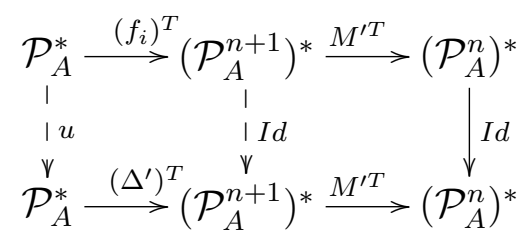

então existe $u \in \mathcal{P}_{A}$ invertível tal que $f_{i}=u \Delta_{i}$, ou seja $I_{A}=J_{A}$.

Assim qualquer deformação de $X=V(\Delta)$ é obtida por $(* *)$.

\subsection{O Grupo $\mathcal{G}(n)$}

Definição 2.3. Para uma matriz $M \in \mathcal{M a t}_{(n+1) n}(\mathcal{P})$ seja $\sum(M)=V(\Delta)$ que é o correspondente germe de espaço analítico em $\left(\mathbf{C}^{m}, 0\right)$, definido pelos menores maximais de $M$.

Definição 2.4. (O Grupo $\mathcal{G}(n)$ ) Seja $G L_{i}(\mathcal{P})$ o grupo das matrizes $i \times i$ invertíveis com entradas em $\mathcal{P}$.

Então definimos $\mathcal{G}(n)=\mathcal{R}_{m} \times G l_{n}(\mathcal{P}) \times G l_{n+1}(\mathcal{P})$, o qual é um grupo com as seguintes operações.

$$
(\phi, R, L) \circ(\psi, S, N)=\left(\phi \circ \psi,\left(\psi^{*} R\right) . S,\left(\psi^{*} L\right) . N\right)
$$

onde $\psi^{*}(M)$ é a matriz gerada aplicando $\psi$ em cada uma das entradas de $M$.

Definimos a ação de $\mathcal{G}(n)$ sobre $\mathcal{M a t}_{(n+1) n}(\mathcal{P})$

$$
\begin{aligned}
\Psi: \mathcal{G}(n) \times \mathcal{M a t}_{(n+1) n}(\mathcal{P}) & \longrightarrow \operatorname{Mat}_{(n+1) n}(\mathcal{P}) \\
((\phi, R, L), M) & \longmapsto L^{-1}\left(\phi^{*} M\right) \cdot R .
\end{aligned}
$$

Dizemos que $M_{1}, M_{2}$ são $\mathcal{G}(n)$-equivalentes se: $\exists(\phi, R, L) \in \mathcal{G}(n)$ tal que $M_{2}=L^{-1}\left(\phi^{*} M_{1}\right) R$.

Uma matriz $M \in \mathcal{M a t}_{n(n+1)}(\mathcal{P})$ pode ser considerada como uma aplicação $f$ : $\mathbb{K}^{m}, 0 \rightarrow \mathbb{K}^{n(n+1)}$. Podemos identificar $\operatorname{Mat}_{n(n+1)}(\mathcal{P}) \operatorname{com} \mathcal{P}^{n(n+1)}$ e o grupo $\mathcal{G}(n)$ pode ser considerado como um subgrupo do grupo de contato correspondente. 
Lema 2.5. O grupo $\mathcal{G}(n)$ age no espaço das aplicações $\left(\mathbb{K}^{m}, 0\right) \rightarrow \mathbb{K}^{n(n+1)}$ como um subgrupo do grupo de contato $\mathcal{K}$.

Demonstração: A ação do grupo $\mathcal{R}_{m}$ em ambos grupos coincidem. O grupo $\mathcal{C}$ é o conjunto de aplicações $\mathbb{K}, 0 \rightarrow G l_{(n+1) n}(\mathbb{K})$. Existe um homomorfismo natural

$$
\begin{aligned}
\theta: G l_{n}(\mathbb{K}) \times G l_{n+1}(\mathbb{K}) & \longrightarrow G l_{n(n+1)}(\mathbb{K}) \\
(R, L) & \longmapsto R \otimes L,
\end{aligned}
$$

onde $R \otimes L$ é o produto de kronecker das matrizes $R$ e $L$, e o determinante é dado por $\operatorname{det}(\theta(R, L))=\operatorname{det}(R \otimes L)=(\operatorname{det}(R))^{n+1}(\operatorname{det}(L))^{n}$. Além disso a aplicação $\theta$ fornece um homomorfismo entre os grupos $G l_{n}(\mathcal{P}) \times G l_{n+1}(\mathcal{P})$ e $\mathcal{C}$, daí segue o resultado.

Vamos exemplificar a prova anterior para o caso $n=2$.

Exemplo 2.6. Sejam $(R, L) \in G l_{2}(\mathcal{P}) \times G l_{3}(\mathcal{P}),(R, L)$ age sobre as aplicações $\mathbb{K}^{m}, 0 \rightarrow \mathbb{K}^{6}$, no mesmo contexto $Z=\theta(R, L) \in \mathcal{C}$, onde:

$$
\begin{gathered}
R=\left(\begin{array}{ll}
a & b \\
c & d
\end{array}\right), \quad L=\left(\begin{array}{ccc}
e & f & g \\
h & i & j \\
k & l & m
\end{array}\right), \\
Z=\left(\begin{array}{cccccc}
a e & a f & a g & c e & c f & c g \\
a h & a i & a j & c h & c i & c j \\
a k & a l & a m & c k & c l & c m \\
b e & b f & b g & d e & d f & d g \\
b h & b i & b j & d h & d i & d j \\
b k & a l & b m & d k & d l & d m
\end{array}\right)
\end{gathered}
$$

O seguinte lema nos garante que a relação de equivalência do grupo $\mathcal{G}(n)$ para matrizes se identifica com a relação de equivalência do grupo $\mathcal{K}$ para funções determinadas pelos seus menores maximais.

Lema 2.7. Sejam $\sum\left(M_{1}\right)=V\left(\Delta^{1}\right)$ e $\sum\left(M_{2}\right)=V\left(\Delta^{2}\right)$ germes de espaços de codimensão 2 correspondentes às matrizes $M_{1}, M_{2} \in \mathcal{M a t}_{n(n+1)}(\mathcal{P})$, onde $\Delta^{i}$ são os ideais gerado pelos menores maximais das matrizes $M_{i}$. Então $\Delta^{1} \sim_{\mathcal{K}} \Delta^{2}$ se, somente se $M_{2} \in \mathcal{G}(n) . M_{1}$.

\section{Demonstração:}

$(\Rightarrow) \Delta^{1} \sim_{\mathcal{K}} \Delta^{2}$, então $\exists \phi \in \mathcal{R}_{m}$ e $A \in G L_{n+1}(\mathcal{P})$ tais que:

$$
\Delta^{2} A=\phi^{*} \Delta^{1}
$$


onde $\Delta^{i}$ são os ideais gerado pelos menores maximais das matrizes $M_{i}$. Pela Proposição (2.1) as seqüências

$$
0 \longrightarrow \mathcal{P}^{n} \stackrel{M_{i}}{\longrightarrow} \mathcal{P}^{n+1} \stackrel{\Delta^{i}}{\longrightarrow} \mathcal{P} \longrightarrow \frac{\mathcal{P}}{\left(\Delta^{i}\right)} \longrightarrow 0
$$

são exatas e o seguinte diagrama é comutativo.

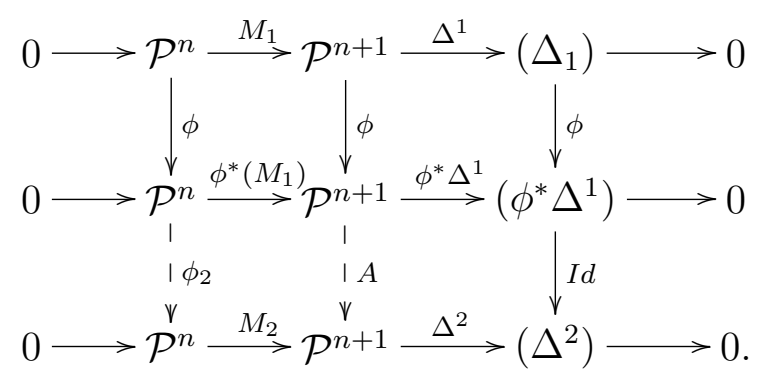

Como a segunda e terceira linhas são exatas e como temos uma aplicação que neste caso é a identidade, então existem aplicações $I d, A, \phi_{2}$ (que são únicas salvo homotopias) tais que o diagrama acima comuta. Como as aplicações do lado direito são isomorfismos então $A, \phi_{2}$ são isomorfismos portanto.

$$
M_{2} \circ \phi_{2}=A \circ \phi^{*}\left(M_{1}\right)
$$

Daí, $M_{2}=\left(A^{-1}\right)^{-1} \phi^{*}\left(M_{1}\right)\left(\phi_{2}^{-1}\right)^{-1}$.

$(\Leftarrow) M_{2} \in \mathcal{G}(n) . M_{1}$ então $\exists L \in G l_{n+1}(\mathcal{P}), R \in G l_{n}(\mathcal{P})$ e $\phi \in \mathcal{R}_{m}$ tal que $M_{2}=$ $L^{-1} \cdot\left(\phi^{*} M_{1}\right) \cdot R$.

Então temos o seguinte diagrama comutativo:

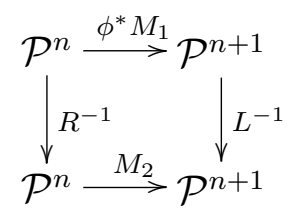

pela Proposição (2.1) temos que as seguintes seqüências são exatas:

$$
0 \longrightarrow \mathcal{P}^{n} \stackrel{M_{i}}{\longrightarrow} \mathcal{P}^{n+1} \stackrel{\Delta^{i}}{\longrightarrow} \mathcal{P} \longrightarrow \frac{\mathcal{P}}{\left(\Delta^{i}\right)} \longrightarrow 0
$$


e temos o seguinte digrama :

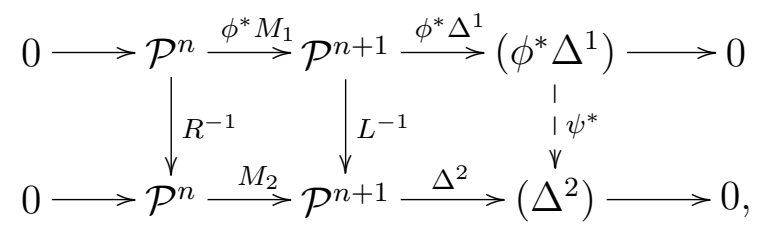

então temos a existência de $\psi^{*}$ que é invertível, e tal que o diagrama acima comuta. Ou seja temos

$$
\begin{aligned}
\psi^{*} \phi^{*}\left(\Delta^{1}\right) & =\Delta^{2} L^{-1} \\
(\phi \psi)^{*}\left(\Delta^{1}\right) & =\Delta^{2} L^{-1}
\end{aligned}
$$

portanto $\Delta^{1} \sim_{\mathcal{K}} \Delta^{2}$

Por cálculo direito podemos mostrar que para $M \in \mathcal{M a t}_{n(n+1)}(\mathcal{P})$, a inclusão definida assim:

$$
\begin{aligned}
& \psi: \operatorname{Mat}_{n(n+1)}(\mathcal{P}) \longrightarrow \operatorname{Mat}_{(n+1)(n+2)}(\mathcal{P}) \\
& M \quad \longmapsto \quad \psi_{\mathcal{M}}(M) \quad=\left(\begin{array}{cc}
M & 0 \\
0 & 1
\end{array}\right)
\end{aligned}
$$

não muda os menores maximais da matriz em $\mathcal{M a t}_{n(n+1)}(\mathcal{P})$ e é compatível com a inclusão definida pelo grupo $\mathcal{G}(n)$.

$$
\begin{aligned}
\psi_{\mathcal{G}}: \mathcal{G}(n) & \longrightarrow \mathcal{G}(n+1) \\
(R, L, \phi) & \longmapsto \psi_{\mathcal{G}}(R, L, \phi)=\left(\left(\begin{array}{cc}
R & 0 \\
0 & 1
\end{array}\right),\left(\begin{array}{ll}
L & 0 \\
0 & 1
\end{array}\right), \phi\right) .
\end{aligned}
$$

Isto é, para todo $M_{1}, M_{2} \in \operatorname{Mat}_{n(n+1)}(\mathcal{P})(n)$ então $M_{2}=L^{-1} \phi^{*}\left(M_{1}\right) R \Longleftrightarrow \psi\left(M_{2}\right)=$ $\psi_{\mathcal{G}}(L)^{-1} \phi^{*} \psi\left(M_{1}\right) \psi_{\mathcal{G}}(R)$.

Além disso para cada matriz $M \in \mathcal{M a t}_{n(n+1)}(\mathcal{P}) \operatorname{com} M_{i j}(0, \ldots, 0) \neq 0$ para alguma entrada $M_{i j}$, existe uma matriz $M_{1} \in \mathcal{M a t}_{n(n-1)}(\mathcal{P})$ tal que $\psi\left(M_{1}\right) \sim_{\mathcal{G}(n)} M$. Pelas transformações de linhas e colunas da matriz $M$, então existe $L \in G l_{n+1}(\mathbb{C}\{x\}), R \in$ $G l_{n}(\mathbb{C}\{x\})$ e $M_{1} \in \mathcal{M a t}_{n \times(n-1)}(\mathbb{C}\{x\})$ tais que:

$$
M=L .\left(\begin{array}{cc}
M_{1} & 0 \\
0 & 1
\end{array}\right) \cdot R .
$$

Além disso o ideal $\Delta$ gerado pelos menores maximais da matriz $M$ é igual ao ideal $\Delta^{\prime}$ 
gerado pelos menores maximais da matriz $M_{1}$. Portanto, sem perda de generalidade podemos considerar matrizes cujas entradas pertencem ao ideal maximal de $\mathcal{P}$.

\subsection{Espaço Tangente}

Como já definimos uma ação sobre o espaço das matrizes $(n+1) \times n$, a primeira tarefa para a classificação é determinar o espaço tangente sob a ação do grupo $\mathcal{G}(n)$. Consideremos o espaço das matrizes com entradas em $\mathcal{P}$ como um $\mathcal{P}$ módulo. $\mathrm{O}$ espaço tangente será considerado como um $\mathcal{P}$ submódulo de $\mathcal{M} t_{(n+1) n}(\mathcal{P})$.

Teorema 2.8. Seja $M \in \mathcal{M a t}_{(n+1) n}(\mathcal{P})$.

1. O espaço tangente com relação ao grupo $\mathcal{R}_{m}$ é dado por:

$$
T_{M} \mathcal{R}_{m}(M)=\mathbf{m}\left\{\frac{\partial M}{\partial x_{i}}, \forall 1 \leq i \leq m\right\} .
$$

Usamos também a notação $J(M)$ para o $\mathcal{P}$ - módulo gerado pelas matrizes da forma

$$
\frac{\partial M}{\partial x_{j}}=\left(\begin{array}{ccc}
\frac{\partial M_{11}}{\partial x_{j}} & \cdots & \frac{\partial M_{1 n}}{\partial x_{j}} \\
\vdots & \ddots & \vdots \\
\frac{\partial M_{(n+1) 1}}{\partial x_{j}} & \cdots & \frac{\partial M_{(n+1) n}}{\partial x_{j}}
\end{array}\right)_{(n+1) n}
$$

$\forall 1 \leq j \leq m$

2. O espaço tangente com relação ao grupo $G=G l_{n+1}(\mathcal{P}) \times G l_{n}(\mathcal{P})$ é dado por:

$$
T_{M} G(M)=\mathcal{P}\left\{(A M+M B) / A \in \mathcal{M a t}_{n+1}(\mathcal{P}), B \in \mathcal{M a t}_{n}(\mathcal{P})\right\}
$$

3. O espaço tangente com relação ao grupo $\mathcal{G}(n)=\mathcal{R}_{m} \times G$ é dado pela soma dos espaços tangentes:

$$
T_{M} \mathcal{G}(M)=\mathbf{m}\left\{\frac{\partial M}{\partial x_{i}}, \forall 1 \leq i \leq m\right\}+\mathcal{P}\left\{(A M+M B) / A \in \mathcal{M a t}_{n+1}(\mathcal{P}), B \in \mathcal{M a t}_{n}(\mathcal{P})\right\}
$$

\section{Demonstração:}


1. Temos então a seguinte ação:

$$
\begin{aligned}
\Upsilon: \mathcal{R}_{m} \times \operatorname{Mat}_{(n+1) n}(\mathcal{P}) & \longrightarrow \operatorname{Mat}_{(n+1) n}(\mathcal{P}) \\
(\phi, M) & \longmapsto \phi^{*}(M) .
\end{aligned}
$$

Vamos usar a Proposição (1.11) para determinar o espaço tangente. Considere-se o seguinte caminho em $\mathcal{R}_{m}$

$$
\begin{aligned}
\alpha: I & \longrightarrow \mathcal{R}_{m} \\
t & \longmapsto I d_{m}+t E_{i j},
\end{aligned}
$$

onde $I d_{m}$ é a matriz identidade $m \times m$ e $E_{i j}$ é a matriz $m \times m$ com 1 na entrada $(i, j)$ e zero em qualquer outro lugar. Temos,

$$
\begin{aligned}
\Upsilon \circ \alpha(t) & =\Upsilon\left(I d_{m}+t E_{i j}\right) \\
& =M\left(I d_{m}+t E_{i j}\right) .
\end{aligned}
$$

Daí:

$$
(\Upsilon \circ \alpha)^{\prime}(t)=\frac{\partial M}{\partial x_{k}} \cdot E_{i j}
$$

portanto temos a primeira afirmação.

2. Temos a seguinte ação:

$$
\begin{aligned}
\Upsilon: G \times \mathcal{M a t}_{(n+1) n}(\mathcal{P}) & \longrightarrow \operatorname{Mat}_{(n+1) n}(\mathcal{P}) \\
(L, R, M) & \longmapsto \text { L.M.R. }
\end{aligned}
$$

Considere-se o seguinte caminho em $G$

$$
\begin{aligned}
\alpha: I & \longrightarrow G \\
t & \longmapsto\left(I d_{n+1}+t A, i d_{n}+t B\right)
\end{aligned}
$$


então:

$$
\begin{aligned}
\Upsilon \circ \alpha(t) & =\Upsilon\left(I d_{n+1}+t A, I d_{n}+t B\right) \\
& =\left(I d_{n+1}+t A\right) M(I d)\left(I d_{n}+t B\right) \\
& =M+t M B+t A M+t^{2} A M B
\end{aligned}
$$

Derivando esta igualdade com respeito a $t$ temos:

$$
(\Upsilon \circ \alpha)^{\prime}(t)=A M+M B
$$

e portanto temos a afirmação (2).

3. Segue dos items (1) e (2).

Definição 2.9. O espaço tangente estendido para $M \in \mathcal{M a t}_{(n+1) n}$ é dado por:

$$
T_{e} \mathcal{G}(n) M=\left\{\langle J(M), A M+M B\rangle / A \in \mathcal{M a t}_{n+1}(\mathcal{P}), B \in \mathcal{M a t}_{n}(\mathcal{P})\right\}
$$

Temos o seguinte resultado para o Grupo $\mathcal{G}(n)$.

Proposição 2.10. (Transversal Completa, [4].) Seja $M$ um k-jato em $J^{k} \mathcal{M a t}_{(n+1) n}(\mathbb{C}\{x\})$, escrevemos $\mathcal{G}_{1}$ para o subgrupo de $\mathcal{G}(n)$ dos elementos cujo 1- jato é a identidade, e $H^{k+1} \mathcal{M a t}_{(n+1) n}(\mathbb{C}\{x\})$ para as aplicações polinomiais homogêneas de grau $k+1$. Suponha que

$$
T_{M} J^{k+1} \mathcal{G}_{1} M \cap H^{k+1} \mathcal{M a t}{ }_{(n+1) n}(\mathbb{C}\{x\})+T=H^{k+1} \mathcal{M a t}_{(n+1) n}(\mathbb{C}\{x\})
$$

para algum subespaço $T$ de $H^{k+1} \mathcal{M a t}_{(n+1) n}(\mathbb{C}\{x\})$. Então todo $(k+1)$ - jato com $k$-jato $M$ é $J^{k+1} \mathcal{G}_{1}$ - equivalente a um jato da forma $M+N$, com $N \in T$. Tal $T$ é chamado de transversal completa.

Observação 2.11. Se $M \in \mathcal{M a t}_{(n+1) n}(\mathbb{C}\{x\})$, temos que

$$
T \mathcal{G}_{1} \cdot M=\mathbf{m}^{2}\left\{\frac{\partial M}{\partial x_{i}}\right\}+\mathbf{m}\left\{A M+M B / A \in \mathcal{M a t}_{n+1}(\mathbb{C}\{x\}), B \in \mathcal{M a t}_{n}(\mathbb{C}\{x\})\right\}
$$




\subsection{O Cálculo de $T^{1}$}

Vimos na Proposição (2.1) que qualquer deformação pode ser expressa como uma perturbação da matriz representação. Nesta seção expressaremos o módulo normal, $N_{X}$, e o espaço das deformações da primeira ordem, $T^{1}$, em termos de matrizes e assim estaremos em condições de tratar a base das deformações semi- universais usando matrizes.

Lema 2.12. Seja $M \in \mathcal{M a t}_{(n+1) n}(\mathcal{P})$ com entradas no ideal maximal $\mathbf{m}_{m}, X$ a variedade definida por seus menores maximais. Então o módulo normal é dado por:

$$
N_{X}=\frac{\mathcal{M a t}_{(n+1) n}(\mathcal{P})}{\operatorname{Img}}
$$

onde

$$
\begin{aligned}
g: \operatorname{Mat}_{(n+1)(n+1)}(\mathcal{P}) \oplus \mathcal{M a t}_{(n) n}(\mathcal{P}) & \longrightarrow \operatorname{Mat}_{(n+1) n}(\mathcal{P}) \\
(A, B) & \longmapsto A M+M B .
\end{aligned}
$$

Demonstração: Para provar o lema é suficiente mostrar que a seguinte seqüência é exata:

$$
\cdots \longrightarrow \operatorname{Mat}_{(n+1)(n+1)}(\mathcal{P}) \oplus \operatorname{Mat}_{(n) n}(\mathcal{P}) \stackrel{g}{\longrightarrow} \operatorname{Mat}_{(n+1)(n)}(\mathcal{P}) \stackrel{\Phi}{\longrightarrow} N_{X} \longrightarrow 0 .
$$

1. Definição de $\Phi$ e prova da sobrejetividade.

Seja

$$
\begin{aligned}
\Phi: \operatorname{Mat}_{(n+1) n}(\mathcal{P}) & \longrightarrow N_{X}=\operatorname{Hom}_{\mathcal{P}}\left(I, \frac{\mathcal{P}}{I}\right) \\
N & \longmapsto \Phi(N),
\end{aligned}
$$

onde

$$
\begin{aligned}
\Phi(N): I & \longrightarrow \frac{\mathcal{P}}{I} \\
\Delta_{k} & \longmapsto \sum_{i=1}^{n+1} \sum_{j=1}^{n}(-1)^{i+j} N_{i j} M_{k, i j},
\end{aligned}
$$

onde $M_{k, i j}$ é o menor sub maximal de $M$ que resulta deixando a i-ésima e k-ésima coluna e j-ésima linha e então formando o determinante.

Tem-se que $\Phi$ está bem definida.

Agora vamos provar a sobrejetividade. Seja $b \in N_{X}$, por Artin ([3]) toda deformação pode ser identificada com a deformação $\left(\Delta_{1}+\epsilon b_{1}, \ldots, \Delta_{n+1}+\epsilon b_{n+1}\right)$, e pela Proposição (2.1) qualquer deformação de um germe Cohen-Macaulay de codimensão 2 pode ser escrita como uma perturbação da matriz $M$. Então existe $N=\left(N_{i j}\right) \in \mathcal{M a t}_{(n+1) n}$ tal que $M+\epsilon N$ é a perturbação de $M$. A correspondente deformação de primeira ordem é dada pelos menores maximais de $M+\epsilon N$ 
módulo $\epsilon^{2}$, ou seja:

$$
\Delta_{k}+\epsilon \sum_{i=1}^{n+1} \sum_{j=1}^{n}(-1)^{i+j} N_{i j} M_{k, i j},
$$

onde $1 \leq k \leq n+1, i \neq k$. Temos então que $\Delta_{k}+\epsilon b_{k}=\Delta_{k}+\epsilon \sum_{i=1}^{n+1} \sum_{j=1}^{n}(-1)^{i+j} N_{i j} M_{k, i j}$ portanto $b_{k}=\sum_{i=1}^{n+1} \sum_{j=1}^{n}(-1)^{i+j} N_{i j} M_{k, i j}$, assim $\Psi(N)=b$.

2. Verificando que a seqüência é um complexo em $\mathcal{M a t}_{(n+1) n}(\mathcal{P})$.

Ou seja, provaremos que $\operatorname{im}(g) \subset \operatorname{ker}(\Phi)$.

De fato, seja $\widetilde{M} \in i m(g)$ então $\exists \widetilde{A} \in \operatorname{Mat}_{(n+1) n}(\mathcal{P}), \widetilde{B} \in \mathcal{M a t}_{(n+1) n}(\mathcal{P})$ tais que : $g(\widetilde{A}, \widetilde{B})=\widetilde{M}$ daí, $\widetilde{M}=\widetilde{A} M+M \widetilde{B}$.

Seja $\Delta=\left(\Delta_{1}, \ldots, \Delta_{n+1}\right)$ os menores maximais de $M$, e $\Delta_{\epsilon}=\left(\Delta_{\epsilon_{1}}, \cdots, \Delta_{\epsilon_{n+1}}\right)$, os menores maximais de $M+\epsilon \widetilde{M}$.

Temos que:

$$
\begin{aligned}
\Delta_{\epsilon_{k}} & =\operatorname{det}\left(M_{i j}+\sum \epsilon A_{i l} M_{l j}+\sum \epsilon M_{i l} B_{l j}\right)_{i \in\{1, \cdots, k-1, k+1, \cdots, n+1\}, j \in\{1, \cdots, n\}} \\
& =\Delta_{k}+\epsilon \sum_{i=1}^{n+1} A_{i i} \Delta_{k}+\epsilon \sum_{i=1}^{n} B_{i i} \Delta_{k}+\epsilon \sum_{i=1}^{n+1} A_{i k} \Delta_{i} \\
& =\left(1+\epsilon \sum_{i=1}^{n+1} A_{i i}+\epsilon \sum_{i=1}^{n} B_{i i} \Delta_{k}+\epsilon \sum_{i=1}^{n+1} A_{i k} \Delta_{i} .\right.
\end{aligned}
$$

Assim $\Delta_{\epsilon}$ e $\Delta$ geram o mesmo ideal em $\frac{\mathbb{C}[\epsilon]}{\left(\epsilon^{2}\right)}\left\{x_{1}, \cdots, x_{m}\right\}$. Logo $\Psi(\widetilde{M}) \in I=\Delta$. Portanto $\widetilde{M} \in \operatorname{ker}(\Phi)$.

3. Verificando que o complexo é exato em $\mathcal{M a t}_{(n+1) n}(\mathcal{P})$.

Ou seja, mostraremos que $\operatorname{ker}(\Phi) \subset i m(g)$. Seja $\widetilde{M} \in \operatorname{Ker}(\Phi), \Delta$ e $\Delta_{\epsilon}$ os ideais gerados pelos menores maximais de $M$ e $M+\epsilon \widetilde{M}$, como $\widetilde{M} \in \operatorname{ker}(\Phi)$, então os ideais $\Delta$ e $\Delta_{\epsilon}$ são iguais e assim temos uma $R$-aplicação $A, \operatorname{com} R=\frac{\mathbb{C}(\epsilon)}{\left(\epsilon^{2}\right)}\left(\left\{x_{1}, \cdots, x_{m}\right\}\right)$ e o seguinte diagrama comutativo.

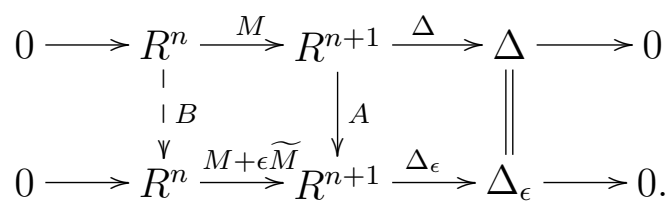

Assim $B$ é um isomorfismo. Além disso sabe-se que : $\left.A^{-1}\right|_{\epsilon=0}=I d_{n+1}$ e $\left.B\right|_{\epsilon=0}=$ $I d_{n}$. 
Temos que $M=M-\left.\epsilon \widetilde{M}\right|_{\epsilon=0}$, além disso

$$
\begin{aligned}
M & =A^{-1}(M-\epsilon \widetilde{M}) B \\
& =\left(I d_{n+1}+\epsilon \widetilde{A}\right)(M-\epsilon \widetilde{M})\left(I d_{n}+\epsilon \widetilde{B}\right) \\
& =M-\epsilon \widetilde{M}+\epsilon \widetilde{A} M+\epsilon M \widetilde{B}
\end{aligned}
$$

então $\widetilde{M}=\widetilde{A} M+M \widetilde{B}$. Portanto $\widetilde{M} \in \operatorname{Im}(g)$.

Lema 2.13. Dada $M \in \mathcal{M a t}_{(n+1) n}(\mathcal{P})$, com as notações do lema anterior,

$$
T_{X}^{1} \cong \frac{\mathcal{M a t}_{(n+1) n}(\mathcal{P})}{J(M)+i m(g)}
$$

Observação 2.14. Dos fatos já mostrados acima temos que :

$$
T_{X}^{1}=\frac{\mathcal{M a t}_{(n+1) n}(\mathcal{P})}{T_{e} \mathcal{G}(n)(M)} .
$$

Demonstração: Pela definição $T_{X}^{1}$ é o co-kernel de:

$$
\begin{aligned}
\Psi: \frac{\mathcal{P}}{(\Delta)} \otimes \Theta & \longrightarrow N_{X} \\
\frac{\partial}{\partial x_{i}} & \longmapsto\left(\frac{\partial \Delta_{1}}{\partial x_{i}}, \cdots, \frac{\partial \Delta_{n+1}}{\partial x_{i}}\right), \quad 0 \leq i \leq m .
\end{aligned}
$$

Pela regra da cadeia temos que:

$$
\begin{aligned}
\frac{\partial \Delta_{k}}{\partial x_{i}} & =\sum_{l=1}^{n+1} \sum_{j=1}^{n} \frac{\partial \Delta_{k}}{\partial M_{l j}} \frac{\partial M_{l j}}{\partial x_{i}}, \quad l \neq k \\
& =\sum_{l=1}^{n+1} \sum_{j=1}^{n}(-1)^{j+l} M_{k, l j} \cdot \frac{\partial M_{l j}}{\partial x_{i}} \\
& =\sum_{l=1}^{n+1} \sum_{j=1}^{n}(-1)^{j+l} \frac{\partial M_{l j}}{\partial x_{i}} M_{k, l j} .
\end{aligned}
$$

E assim é a k-ésima componente de: $\Phi\left(\frac{\partial M_{l j}}{\partial x_{i}}\right)$ na prova do Lema 2.12). Usando o isomorfismo de Lema (2.12) e a sobrejetividade de $\Phi$ podemos definir a aplicação como 
classes de matrizes.

$$
\begin{aligned}
\Psi: \frac{\mathcal{P}}{(\Delta)} \otimes \Theta & \longrightarrow \frac{\operatorname{Mat}_{(n+1) n}(\mathcal{P})}{\operatorname{Im}(g)}\left(\cong N_{X}\right) \\
\frac{\partial}{\partial x_{i}} & \longmapsto\left[\left(\frac{\partial M_{l j}}{\partial x_{i}}\right)\right]_{0 \leq l \leq n+1,0 \leq j \leq n} .
\end{aligned}
$$

Agora calculemos a Imagem de $\Psi$

$$
\operatorname{Im}(\Psi)=\left\{\frac{S}{\operatorname{Im}(g)} /\left[\left(\frac{\partial M_{l j}}{\partial x_{i}}\right)\right]=[(S)]\right\}
$$

portanto $\frac{\partial M_{l j}}{\partial x_{i}}-S \in \operatorname{Im}(g), \operatorname{assim} S \in \operatorname{Im}(g)+J(M)$

$$
\operatorname{Im}(\Psi)=\frac{J(M)+\operatorname{Im}(g)}{\operatorname{Im}(g)}
$$

Portanto, $T_{X}^{1} \cong \frac{\frac{\mathcal{M a t}_{(n+1) n}(\mathcal{P})}{\operatorname{Im}(g)}}{\frac{J(M)+\operatorname{Im}(g)}{\operatorname{Im}(g)}} \cong \frac{\mathcal{M a t}_{(n+1) n}(\mathcal{P})}{J(M)+\operatorname{Im}(g)}$, pelo Teorema de isomorfismo.

Observação 2.15. Quando usamos $T_{X}^{1}$ na notação do lema, usaremos a notação de $T^{1}(M)$. Usando a matriz de pesos relativos, $T^{1}(M)$ pode ser considerado um módulo graduado sobre o anel graduado $\mathcal{P}$ :

$$
T^{1}(M)=\bigoplus_{v \in \mathbb{Z}} T_{v}^{1}(M), T_{v}^{1}(M) \subset G_{v}^{D, a}, \text { ver página } 52 \text {. }
$$

Exemplo 2.16. Seja $A=\left(\begin{array}{cc}z & x \\ x y & z \\ x^{2} & y\end{array}\right)$.

Considere $a=(1,1,1)$ e $D=\left(\begin{array}{ll}1 & 1 \\ 1 & 1 \\ 1 & 1\end{array}\right)$, podemos verificar por cálculos explícitos que uma base para $T^{1}(M)$ é: 


$$
\underbrace{\left(\begin{array}{ll}
0 & 0 \\
0 & 1 \\
0 & 0
\end{array}\right),\left(\begin{array}{ll}
0 & 0 \\
1 & 0 \\
0 & 0
\end{array}\right),\left(\begin{array}{ll}
0 & 0 \\
0 & 0 \\
1 & 0
\end{array}\right)}_{T_{0}^{1}(M)}, \underbrace{\left(\begin{array}{ll}
0 & 0 \\
0 & x \\
0 & 0
\end{array}\right),\left(\begin{array}{ll}
0 & 0 \\
x & 0 \\
0 & 0
\end{array}\right),\left(\begin{array}{ll}
0 & 0 \\
y & 0 \\
0 & 0
\end{array}\right),\left(\begin{array}{ll}
0 & 0 \\
0 & 0 \\
x & 0
\end{array}\right)}_{T_{1}^{1}(M)}, \underbrace{\left(\begin{array}{ll}
0 & 0 \\
x^{2} & 0 \\
0 & 0
\end{array}\right)}_{T_{2}^{1}(M)} .
$$

portanto $T^{1}(M)=T_{0}^{1}(M) \oplus T_{1}^{1}(M) \oplus T_{2}^{1}(M)$.

Definição 2.17. Dizemos que $M \in \operatorname{Mat}_{(n+1) n}(\mathcal{P})$ tem codimensão finita se

$$
\operatorname{cod}_{\mathcal{G}(n)} M=\operatorname{dim}_{\mathbb{K}} T^{1}(M)<\infty
$$

Definição 2.18. Seja $M \in \mathcal{M} a t(\mathbb{C}\{x\})$. Definamos o número de Tjurina de $M$, denotado por $\tau$, como sendo a seguinte dimensão

$$
\tau(M)=\operatorname{dim}_{\mathbb{C}} T^{1}(M) .
$$

Exemplo 2.19. Seja $M=\left(\begin{array}{c}x^{2}+y^{2}+z^{3} \\ y z\end{array}\right) \in \mathcal{M a t}_{2 \times 1}(\mathbb{C}\{x, y, z\})$ então temos que o espaço tangente à orbita de $M$ é gerado por:

$$
\left(\begin{array}{l}
x \\
0
\end{array}\right),\left(\begin{array}{c}
2 y \\
z
\end{array}\right),\left(\begin{array}{c}
3 z^{2} \\
y
\end{array}\right),\left(\begin{array}{c}
x^{2}+y^{2}+z^{3} \\
0
\end{array}\right),\left(\begin{array}{c}
0 \\
x^{2}+y^{2}+z^{3}
\end{array}\right),\left(\begin{array}{c}
y z \\
0
\end{array}\right),\left(\begin{array}{c}
0 \\
y z
\end{array}\right),
$$

logo uma base para $T^{1}(M)$ é:

$$
\left(\begin{array}{l}
1 \\
0
\end{array}\right),\left(\begin{array}{l}
0 \\
1
\end{array}\right),\left(\begin{array}{l}
0 \\
x
\end{array}\right),\left(\begin{array}{l}
y \\
0
\end{array}\right),\left(\begin{array}{l}
z \\
0
\end{array}\right),\left(\begin{array}{l}
0 \\
z
\end{array}\right),
$$

pois:

$$
\begin{aligned}
\left(\begin{array}{l}
0 \\
y
\end{array}\right) & =\left(\begin{array}{c}
3 z^{2} \\
y
\end{array}\right)-3\left(\begin{array}{c}
x^{2}+y^{2}+y^{2}+z^{2} \\
0
\end{array}\right)+3 x\left(\begin{array}{c}
x \\
0
\end{array}\right)+\frac{3}{2} y\left(\begin{array}{c}
2 y \\
z
\end{array}\right)-\frac{3}{2}\left(\begin{array}{c}
0 \\
y z
\end{array}\right) \\
\left(\begin{array}{c}
0 \\
x z
\end{array}\right) & =x\left(\begin{array}{c}
2 y \\
z
\end{array}\right)-2 y\left(\begin{array}{l}
x \\
0
\end{array}\right) \\
\left(\begin{array}{c}
z^{2} \\
0
\end{array}\right) & =\frac{1}{3}\left(\begin{array}{c}
3 z^{2} \\
y
\end{array}\right)-\frac{1}{3}\left(\begin{array}{l}
0 \\
y
\end{array}\right) \\
\left(\begin{array}{c}
0 \\
z^{2}
\end{array}\right) & =z\left(\begin{array}{c}
2 y \\
z
\end{array}\right)-2\left(\begin{array}{c}
y z \\
0
\end{array}\right) \\
\left(\begin{array}{c}
0 \\
x^{2}
\end{array}\right) & =\left(\begin{array}{c}
0 \\
x^{2}+y^{2}+z^{2}
\end{array}\right)-y\left(\begin{array}{l}
0 \\
y
\end{array}\right)-\left(\begin{array}{c}
0 \\
z^{2}
\end{array}\right),
\end{aligned}
$$


portanto $\tau=6$.

\subsection{Determinação Finita da $\mathcal{G}(n)$ - Equivalência}

Nesta seção apresentaremos critérios de determinação finita do grupo $\mathcal{G}(n)$. O critério infinitesimal é obtido como aplicação do lema da trivialidade local. Além disso apresentamos uma outra prova deste critério infinitesimal de determinação finita usando o Teorema abc de Hironaka [25] em uma nova versão feita por D. Van Straten e Konrad Möhring em seu trabalho [27].

Definição 2.20. Seja $M \in \mathcal{M a t}_{(n+1) n}(\mathcal{P})$. Dizemos que $M$ é $k-\mathcal{G}(n)$ determinado se para toda matriz $N \in \mathcal{M a t}_{(n+1) n}(\mathcal{P})$ tal que $j^{k}(M)=j^{k}(N)$ se tem que $M \sim_{\mathcal{G}(n)} N$.

Dizemos que $M$ é finitamente determinado se $\exists k \in \mathbb{N}$ tal que $M$ é $k-\mathcal{G}(n)$ determinado.

Na proposição seguinte, obtemos uma condição necessaria para a $\mathcal{G}(n)$-determinação finita.

Proposição 2.21. Seja $M \in \mathcal{M a t}_{n(n+1)}(\mathcal{P})$. Se $M$ é $k-\mathcal{G}(n)$ determinado então $\mathbf{m}^{k+1} \mathcal{M a t}_{n(n+1)}(\mathcal{P})$ $\subseteq T \mathcal{G}(n) M$

Demonstração: Como $M$ é finitamente determinado então existe $k \in \mathbb{N}$ tal que $M$ é $k-\mathcal{G}(n)$ determinado. Seja $N \in \mathcal{M a t}_{(n+1) n}(\mathcal{P})$ tal que $j^{k} M=j^{k} N$, queremos mostrar que $M \sim_{\mathcal{G}(n)} N$. Sem perda de generalidade podemos supor que $M \in J^{k} \operatorname{Mat}_{n(n+1)}(\mathcal{P})$. Consideremos a seguinte projeção linear:

$$
\begin{array}{ccc}
\phi: J^{k+1} \mathcal{M a t}_{n(n+1)}(\mathcal{P}) & \longrightarrow & J^{k} \operatorname{Mat}_{n(n+1)}(\mathcal{P}) \\
N & \longmapsto & j^{k} N
\end{array}
$$

A aplicação $\phi$ é linear e sobrejetora portanto a derivada, $d(\phi)=\phi$. Como $M \in J^{k} \mathcal{M a t}_{n(n+1)}(\mathcal{P})$ então $\phi^{-1}(M) \subseteq J^{k+1} \mathcal{M a t}_{n(n+1)}(\mathcal{P})$ é subvariedade de $J^{k+1} \mathcal{M a t}_{n(n+1)}(\mathcal{P})$. O espaço tangente de $\phi^{-1}(M)$ é o núcleo de $\phi$.

$\operatorname{Ker} \phi=\{$ polinômios homogêneos de grau $\mathrm{k}+1\}=\frac{\mathbf{m}^{k+1} \mathcal{M} a t_{n(n+1)}(\mathcal{P})}{\mathbf{m}^{k+2} \mathcal{M a t}_{n(n+1)}(\mathcal{P})}$.

Afirmação: $\phi^{-1}(M) \subset J^{k+1} \mathcal{G}(n) M$.

De fato, dado $N \in \phi^{-1}(M)$ então $j^{k} N=M$. Como $M$ é $k-\mathcal{G}(n)$ determinado segue que $N$ é $\mathcal{G}(n)$ equivalente a $M$. Assim existem $(\phi, R, S) \in \mathcal{G}(n)$ tais que $N=R\left(\phi^{*} M\right)$.S. Logo: $j^{k+1} N=N=j^{k+1}\left(R\left(\phi^{*} M\right) . S\right) \in J^{k+1} \mathcal{G}(n) M$, então $N \in J^{k+1} \mathcal{G}(n) M$ e assim $\phi^{-1}(M) \subset J^{k+1} \mathcal{G}(n) M$, o que prova a afirmação.

Logo : 


$$
\frac{\mathbf{m}^{k+1} \mathcal{M a t}_{n(n+1)}(\mathcal{P})}{\mathbf{m}^{k+2} \mathcal{M} t_{n(n+1)}(\mathcal{P})} \cong T\left(\phi^{-1} M\right) \subset T J^{K+1} \mathcal{G}(n) M=\frac{T \mathcal{G} M+\mathbf{m}^{k+2} \mathcal{M a t}_{n(n+1)}(\mathcal{P})}{\mathbf{m}^{k+2} \mathcal{M a t}_{n(n+1)}(\mathcal{P})}
$$

$\operatorname{assim} \mathbf{m}^{k+1} \mathcal{M a t}{ }_{(n+1) n}(\mathcal{P}) \subseteq T \mathcal{G} M+\mathbf{m}^{k+2} \mathcal{M a t}{ }_{(n+1) n}(\mathcal{P})$.

Pelo Lema de Nakayama (1.14) temos que: $\mathbf{m}^{k+1} \mathcal{M a t}_{n(n+1)}(\mathcal{P}) \subseteq T \mathcal{G}(n) M$

Apresentaremos a seguir o seguinte lema o qual é uma generalização do Teorema (1.20) para matrizes. Este lema trata de singularidades Cohen-Macaulay de codimensão dois em termos da matriz representação.

Lema 2.22. (Trivialidade local) Seja $c \geq 0$ um inteiro e $M \in \mathcal{M a t}_{(n+1) n}\left(\mathbb{C}\left\{x_{1}, \ldots, x_{m}, y_{1} \ldots, y_{k}\right\}\right)$ que define uma família de singularidades isoladas Cohen-Macaulay de codimensão 2, onde os $y_{i}$ são considerados os parâmetros. Então as seguintes afirmações são equivalentes:

1. $\frac{\partial M}{\partial y_{j}} \in\left(x_{1}, \ldots, x_{m}\right)^{c}\left(\frac{\partial M}{\partial x_{1}}, \ldots, \frac{\partial M}{\partial x_{m}}\right)+\operatorname{Im}(g), \quad \forall 0 \leq j \leq k$ onde g é a aplicação definida no Lema (2.12).

2. Existem $\phi_{1}, \ldots, \phi_{m} \in \mathbb{C}\left\{x_{1}, \ldots, x_{m}, y_{1} \ldots, y_{k}\right\}, A \in \mathcal{M a t}_{(n+1)(n+1)}\left(\mathbb{C}\left\{x_{1}, \ldots, x_{m}, y_{1} \ldots, y_{k}\right\}\right)$ e $B \in \mathcal{M a t}_{n \times n}\left(\mathbb{C}\left\{x_{1}, \ldots, x_{m}, y_{1} \ldots, y_{k}\right\}\right)$ tais que:

- $A\left(x_{1}, \ldots, x_{n}, 0, \ldots, 0\right)=I d_{n+1}$

- $B\left(x_{1}, \ldots, x_{n}, 0, \ldots, 0\right)=I d_{n}$

- $\phi_{i}\left(x_{1}, \ldots, x_{n}, 0, \ldots, 0\right)=x_{i}, \forall 0 \leq i \leq m$

- $\phi_{i}-x_{i} \in\left(x_{1}, \ldots, x_{m}\right)^{c}, \forall 0 \leq i \leq m$

- $A \cdot M\left(x_{1}, \ldots, x_{m}, y_{1} \ldots, y_{k}\right) \cdot B=M\left(\phi_{1}, \ldots, \phi_{m}, 0 \ldots, 0\right)$.

\section{Demonstração:}

$(2) \Longrightarrow(1)$ Temos por hipótese que $\phi_{i}\left(x_{1}, \ldots, x_{n}, 0, \ldots, 0\right)=x_{i}, \forall 1 \leq i \leq m$ então definamos:

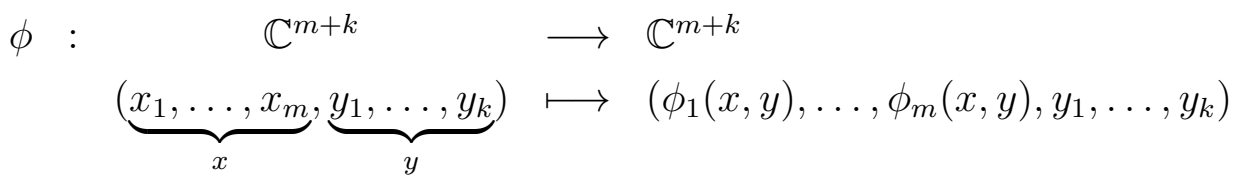

que é invertível. Denotemos $\psi=\phi^{-1}$ e ponhamos $\psi=\left(\psi_{1}, \ldots, \psi_{m}, y_{1}, \ldots, y_{k}\right)$ Afirmamos que: $\psi_{i}-x_{i} \in(x)^{c}, \forall 1 \leq i \leq m$. De fato, temos por hipótese que: $\phi_{i}-x_{i} \in\langle x\rangle^{c}$, então $\phi_{i}-x_{i}=\xi_{i} \operatorname{com} \xi \in\langle x\rangle^{c}$ assim $\phi_{i}(x, y)=\xi_{i}+x_{i}$, então

$$
\begin{aligned}
\psi\left(\phi_{1}, \ldots, \phi_{m}, y\right) & =\left(\psi_{1}\left(\xi_{1}+x_{1}, \ldots, \xi_{m}+x_{m}\right), \ldots, \psi_{m}\left(\xi_{1}+x_{1}, \ldots, \xi_{m}+x_{m}\right), y\right) \\
(x, y) & =\left(\psi_{1}\left(\xi_{1}+x_{1}, \ldots, \xi_{m}+x_{m}\right), \ldots, \psi_{m}\left(\xi_{1}+x_{1}, \ldots, \xi_{m}+x_{m}\right), y\right)
\end{aligned}
$$


daí: $\psi_{i}\left(\xi_{1}+x_{1}, \ldots, \xi_{m}+x_{m}\right)=x_{i}$. Fazendo mudança de variáveis temos que: $\psi_{i}-x_{i} \in\langle x\rangle^{m}$, o que mostra a afirmação.

Por outro lado pela hipótese temos que $A \cdot M(x, y) \cdot B=M\left(\phi_{1}, \ldots, \phi_{m}, 0, \ldots, 0\right)$, compondo com $\psi$ teríamos.

$$
A(\psi) \cdot M(x, y) \cdot B(\psi)=M(x, 0) .
$$

Como a equação do lado direito não depende de $y$, derivamos a equação com respeito a $y_{i}$ obtemos:

$$
\frac{\partial}{\partial y_{i}} A(\psi) \cdot M(x, y) \cdot B(\psi)=0
$$

Usando a regra de produto e a regra da cadeia para derivadas tem-se:

$$
\begin{gathered}
\left(\frac{\partial A(\psi)}{\partial y_{i}}+\sum_{j=1}^{m} \frac{\partial A(\psi)}{\partial x_{j}} \cdot \frac{\partial \psi_{j}}{\partial y_{i}}\right) M(\psi) B(\psi)+A(\psi)\left(\frac{\partial M(\psi)}{\partial y_{i}}+\sum_{j=1}^{m} \frac{\partial M(\psi)}{\partial x_{j}} \cdot \frac{\partial \psi_{j}}{\partial y_{i}}\right) B(\psi)+ \\
A(\psi) M(\psi)\left(\frac{\partial B(\psi)}{\partial y_{i}}+\sum_{j=1}^{m} \frac{\partial B(\psi)}{\partial x_{j}} \cdot \frac{\partial \psi_{j}}{\partial y_{i}}\right)=0
\end{gathered}
$$

onde $\frac{\partial \psi_{j}}{\partial y_{i}}$ é a matriz que resulta do produto de cada entrada da matriz com $\frac{\partial \psi_{j}}{\partial y_{i}}$. Compondo com $\phi$ e multiplicando por $A^{-1}$ à esquerda e $B^{-1}$ à direita obtemos:

$$
\begin{aligned}
& \underbrace{A^{-1}\left(\frac{\partial A}{\partial y_{i}}+\sum_{j=1}^{m} \frac{\partial A}{\partial x_{j}} \cdot \frac{\partial \psi_{j}(\phi)}{\partial y_{i}}\right) M}_{\in \operatorname{Im}(g)}+(\frac{\partial M}{\partial y_{i}}+\sum_{j=1}^{m} \frac{\partial M}{\partial x_{j}} \cdot \underbrace{\frac{\partial \psi_{j}(\phi)}{\partial y_{i}}}_{\in\langle x\rangle^{c}})+ \\
& \underbrace{M\left(\frac{\partial B}{\partial y_{i}}+\sum_{j=1}^{m} \frac{\partial B}{\partial x_{j}} \cdot \frac{\partial \psi_{j}(\phi)}{\partial y_{i}}\right) \cdot B^{-1}}_{\in \operatorname{Im}(g)}=0 .
\end{aligned}
$$

Portanto $\frac{\partial M}{\partial y_{i}} \in\langle x\rangle^{c}\left(\frac{\partial M}{\partial x_{i}}, \ldots, \frac{\partial M}{\partial x_{m}}\right)+\operatorname{Im}(g)$.

$(1) \Longrightarrow(2)$ A prova será feita por indução sobre o número de parâmetros.

Para o caso de zero parâmetros considere $A^{(0)}=I d_{n+1}, B^{(0)}=I d_{n}$, e $\phi^{(0)}=\left(x_{1}, \ldots, x_{n}\right)$.

Para o caso de $l$ parâmetros: Suponha que para $M \in \mathcal{M a t}(\mathbb{C}\{x, y\})$ com $x=\left(x_{1}, \ldots, x_{m}\right)$ e $y=\left(y_{1}, \ldots, y_{l}\right)$ existem $A^{(l)} \in \mathcal{M a t}_{n+1}(\mathbb{C}\{x, y\})$, $B^{(l)} \in \operatorname{Mat}_{n}(\mathbb{C}\{x, y\}), \phi_{i} \in \mathbb{C}\{x, y\}, \forall 1 \leq i \leq m$ tal que se cumprem as 5 
condições de (2).

Vamos agora considerar $M\left(x, y, y_{l+1}\right) \in \mathcal{M a t}_{(n+1) n}\left(\mathbb{C}\left\{x, y, y_{l+1}\right\}\right)$. Como $M(x, y, 0) \in$ $\operatorname{Mat}(\mathbb{C}\{x, y\})$, tem $l$ parâmetros, então pela hipótese indutiva existem $A^{(l)} \in$ $\mathcal{M a t}_{n+1}(\mathbb{C}\{x, y\}), B^{(l)} \in \mathcal{M a t}_{n}(\mathbb{C}\{x, y\})$ e $\phi_{i} \in \mathbb{C}\{x, y\}$, tais que:

$$
\begin{aligned}
& -A^{(l)}(x, 0)=I d_{n+1}, \\
& \text { - } B^{l}(x, 0)=I d_{n}, \\
& \text { - } \phi_{i}^{l}=x_{i} \\
& \text { - } \phi_{i}^{l}-x_{i} \in\langle x\rangle^{c}, \\
& \text { - } A^{(l)}(x, y) \cdot M(x, y, 0) \cdot B^{(l)}(x, y)=M\left(\phi_{1}^{l}, \ldots, \phi_{m}^{l}, 0\right) .
\end{aligned}
$$

Pela hipótese (1), temos que: $\exists \xi_{1}, \ldots, \xi_{n} \in\langle x\rangle^{c}\left(\mathbb{C}\left\{x, y, y_{l+1}\right\}\right), L \in \mathcal{M a t}_{n+1}\left(\mathbb{C}\left\{x, y, y_{l+1}\right\}\right)$ e $R \in \mathcal{M a t}_{n}\left(\mathbb{C}\left\{x, y, y_{l+1}\right\}\right)$ tais que:

$$
\frac{\partial M}{\partial y_{l+1}}=L M+M R+\sum_{i=1}^{m} \xi_{i} \cdot \frac{\partial M}{\partial x_{i}} .
$$

Considere o seguinte campo vetorial:

$$
\delta=-\frac{\partial}{\partial y_{l+1}}+\sum_{i=1}^{m} \xi_{i} \frac{\partial}{\partial x_{i}} .
$$

Introduzimos uma nova variável $t$ e definamos um automorfismo.

$$
\begin{aligned}
\Phi: \mathbb{C}\left\{x, y, y_{l+1}, t\right\} & \longrightarrow \mathbb{C}\left\{x, y, y_{l+1}, t\right\} \\
a & \longmapsto \sum_{v=0}^{\infty} \frac{1}{v !} \delta^{v}(a) t^{v}, \quad \forall a \in \mathbb{C}\left\{x, y, y_{l+1}, t\right\} \\
t & \longmapsto t .
\end{aligned}
$$

Em particular,

$$
\begin{aligned}
\Phi_{i}=\phi\left(x_{i}\right) & =x_{i}+\xi_{i} t+\text { termos de ordem maior em } \mathrm{t}, \quad 1 \leq i \leq m \\
\Phi\left(y_{j}\right) & =y_{j}, \quad 1 \leq j \leq l \\
\Phi\left(y_{l+1}\right) & =y_{l+1}-t
\end{aligned}
$$


Além disso, a derivada parcial com respeito a $t$ é:

$$
\begin{aligned}
\frac{\Phi_{i}}{\partial t} & =\sum_{v=1}^{\infty} \frac{1}{v !} \delta^{v}\left(x_{i}\right) v t^{v-1} \\
& =\sum_{v=0}^{\infty} \frac{1}{v !} \delta^{v+1}\left(x_{i}\right) v t^{v} \\
& =\Phi\left(\delta\left(x_{i}\right)\right), \\
& =\xi\left(\Phi_{1}, \ldots, \Phi_{m}, y, y_{l+1}-t\right)
\end{aligned}
$$

Usando esta igualdade, e derivando $M$ com respeito a $t$ tem-se:

$$
\begin{aligned}
\frac{\partial M_{i j}(\underline{\Phi}, \underline{Y})}{\partial t} & =(\sum_{v=1}^{m} \frac{\partial M_{i j}}{\partial x_{v}}(\underline{\Phi}, \underline{Y}) \cdot \underbrace{\frac{\partial \Phi_{v}}{\partial t}(x, \underline{Y})}_{\xi_{v}(\underline{\Phi}, \underline{Y})}-\frac{\partial M_{i j}}{\partial y_{l+1}}(\underline{\Phi}, \underline{Y})) \\
& =\delta\left(M_{i j}\right)(\underline{\Phi}, \underline{Y}) \\
& =\delta(M)_{i j}(\underline{\Phi}, \underline{Y}) \\
& =-(L M+M R)_{i j}(\underline{\Phi}, \underline{Y})
\end{aligned}
$$

onde $\underline{\Phi}=\Phi_{1}, \ldots, \Phi_{m}$ e $\underline{Y}=(\underbrace{0}_{l-v e z e s}, y_{l+1}-t)$.

Para o próximo passo, teremos que encontrar uma matriz $N=N(x, y, t) \in$ $G l_{n+1}(\mathbb{C}\{x, y, t\})$ e $S=S(x, y, t) \in G l_{n}(\mathbb{C}\{x, y, t\})$ com a condição que $N(x, y, 0)=$ $I d_{n+1}$ e $S(x, y, 0)=I d_{n}$ tais que o seguinte sistema de equações diferenciais é satisfeito para $\widetilde{M}=M\left(x, 0, y_{l+1}\right)$ :

$$
\frac{\partial(N \cdot \widetilde{M} \cdot S)_{i j}}{\partial t}=-(L(\underline{\Phi}, \underline{Y}) \cdot N \cdot \widetilde{M} \cdot S+N \cdot \widetilde{M} \cdot S \cdot R(\underline{\Phi}, \underline{Y}))_{i j} .
$$

Usando a regra da cadeia e do produto e o fato que $\widetilde{M}$ é independente de $t$, temos que a equação diferencial é equivalente às seguintes equações diferenciais,

$$
\begin{aligned}
& \frac{\partial N_{i j}}{\partial t}=-(L(\underline{\Phi}, \underline{Y}) \cdot N)_{i j}, \\
& \frac{\partial S_{i j}}{\partial t}=-(S \cdot R(\underline{\Phi}, \underline{Y}))_{i j},
\end{aligned}
$$

com as mesmas condições iniciais mencionadas acima. Então temos a existência e unicidade das soluções da equação diferencial. Assim temos que $M(\underline{\Phi}, \underline{Y})=$ 
$N . \widetilde{M} . S$. Agora pondo $t=y_{l+1}$ dá o seguinte:

$$
M(\underline{\widetilde{\Phi}}, 0)=N\left(x, y, y_{l+1}, y_{l+1}\right) \cdot \widetilde{M} \cdot S\left(x, y, y_{l+1}, y_{l+1}\right)
$$

onde $\underline{\widetilde{\Phi}}$ denota $\underline{\Phi}$ substituindo $t=y_{l+1}$.

Considere $A^{(l+1)}=N\left(x, y, y_{l+1}, y_{l+1}\right) \cdot A^{(l)}, B^{(l+1)}=B^{l} \cdot S\left(x, y, y_{l+1}, y_{l+1}\right)$ e $\phi^{(l+1)}=$ $\underline{\widetilde{\Phi}} \circ \phi^{(l)}$.

Temos então,

$$
\begin{aligned}
& -A^{(l+1)}(x, 0)=N(x, 0) \cdot A^{(l)}(x, 0)=I d_{n+1} \\
& -B^{(l+1)}(x, 0)=B^{(l)}(x, 0) \cdot S(x, 0)=I d_{n} \\
- & A^{(l+1)} M \cdot B^{(l+1)}=N\left(x, y, y_{l+1}, y_{l+1}\right) A^{(l)}(x, y) \cdot M \cdot B^{(l)} S\left(x, y, y_{l+1}, y_{l+1}\right)= \\
& N\left(x, y, y_{l+1}, y_{l+1}\right) \cdot M\left(\phi_{1}^{(l)}, \ldots, \phi_{m}^{(l)}, 0\right) S\left(x, y, y_{l+1}, y_{l+1}\right)=M\left(\underline{\Phi} \circ \phi^{(l)}, 0\right) .
\end{aligned}
$$

O critério infinitesimal da determinação finita é obtido como corolário do Lema da trivialidade local (2.22).

Lema 2.23. (Critério Infinitesimal da determinação Finita.) Seja $M \in \mathcal{M a t}(\mathbb{C}\{x\})$ um germe de matriz. Seja $k>0$ um inteiro tal que:

$$
\mathbf{m}^{k+1} \mathcal{M a t}_{(n+1) n}(\mathbb{C}\{x\}) \subset \mathbf{m}^{2}\left(\frac{\partial M}{\partial x_{1}}, \ldots \frac{\partial M}{\partial x_{m}}\right)+\mathbf{m} \operatorname{Im}(g) .
$$

Então $M$ é $k$ - finitamente determinado.

Demonstração: Para a prova basta mostrar que para qualquer perturbação $M+N$ de $M$ cujas entradas $N_{i j} \in \mathbf{m}^{k+1}$ tem-se que $M+N \sim_{\mathcal{G}(n)} M$.

Seja $N \in \mathbf{m}^{k+1} \mathcal{M a t}(\mathbb{C}\{x\})$, e considere matrizes da forma $M_{a}=M+a N$ para $0 \leq a \leq 1$.

1. A hipótese geral (2.2) também vale para $M_{a}$, ou seja, temos que mostrar que

$$
\mathbf{m}^{k+1} \mathcal{M a t}_{(n+1) n}(\mathbb{C}\{x\}) \in \mathbf{m}^{2}\left(\frac{\partial M_{a}}{\partial x_{1}}, \ldots \frac{\partial M_{a}}{\partial x_{m}}\right)+\mathbf{m} \operatorname{Im}\left(g_{a}\right),
$$

onde $g_{a}$ é a aplicação já definida no Lema (2.12), substituindo $M$ por $M_{a}$. Seja $\widetilde{N} \in \mathbf{m}^{k+1} \mathcal{M a t}_{(n+1) n}(\mathbb{C}\{x\})$, temos que provar que $\exists \xi_{i} \in \mathbf{m}^{2}, A \in \operatorname{Mat}_{n+1}(\mathbb{C}\{x\})$, 
$B \in \mathcal{M a t}_{n}(\mathbb{C}\{x\}) \operatorname{com} A_{i j}, B_{i j} \in \mathbf{m}$ tais que:

$$
\widetilde{N}=\sum_{i=1}^{m} \xi_{i} \frac{\partial M_{a}}{\partial x_{i}}+A \cdot M_{a}+M_{a} \cdot B .
$$

Como $\widetilde{N} \in \mathbf{m}^{k+1} \mathcal{M a t}_{(n+1) n}(\mathbb{C}\{x\})$, então pela hipótese temos que

$$
\widetilde{N}=\sum_{i=1}^{m} \widetilde{\xi}_{i} \frac{\partial M}{\partial x_{i}}+\widetilde{A} \cdot M+M \cdot \widetilde{B}
$$

onde $\widetilde{\xi}_{i} \in \mathbf{m}^{2}$ e $\widetilde{A}_{i j}, \widetilde{B}_{i j} \in \mathbf{m} \forall i, j$. Substituindo $M=M_{a}-a N$ temos

$$
\widetilde{N}=\sum_{i=1}^{m} \widetilde{\xi}_{i} \frac{\partial M_{a}}{\partial x_{i}}+\widetilde{A} \cdot M_{a}+M_{a} \cdot \widetilde{B}-\underbrace{\left(\sum_{i=1}^{m} a \widetilde{\xi}_{i} \frac{\partial N}{\partial x_{i}}+a \cdot \widetilde{A} \cdot N+a N \cdot \widetilde{B}\right)}_{N^{(1)}} .
$$

Como $N_{i j} \in \mathbf{m}^{k+1}$ então $\frac{\partial N_{i j}}{\partial x_{k}} \in \mathbf{m}^{k}$ e assim $\frac{\partial N}{\partial x_{i}} \in \mathbf{m}^{k}$, portanto $\widetilde{\xi}_{i} \cdot \frac{\partial N}{\partial x_{i}} \in \mathbf{m}^{k+2}$. Assim também as entradas de $\widetilde{A} \cdot N$ e $N . \widetilde{B}$ estão em $\mathbf{m}^{k+2}$.

Novamente pela hipótese sobre $N^{(1)}$ temos:

$$
N^{(1)}=\sum_{i=1}^{m} \xi_{i}^{(1)} \frac{\partial M}{\partial x_{i}}+A^{(1)} \cdot M+M \cdot B^{(1)} .
$$

Com $\xi_{i}^{(1)} \in \mathbf{m}^{3}$ e $A_{i j}^{(1)}, B_{i j}^{(1)} \in \mathbf{m}^{2}$. Substituindo em (2.4) temos:

$$
\begin{aligned}
\widetilde{N}= & \sum_{i=1}^{m}\left(\widetilde{\xi}_{i}+\xi_{i}^{(1)}\right) \frac{\partial M_{a}}{\partial x_{i}}+\left(\widetilde{A}+A^{(1)}\right) \cdot M_{a}+M_{a} \cdot\left(\widetilde{B}+B^{(1)}\right)- \\
& \underbrace{\left(\sum_{i=1}^{m} a \xi^{(1)} \frac{\partial N}{\partial x_{i}}+a A^{(1)} \cdot N+a N \cdot B^{(1)}\right)}_{N^{(2)}},
\end{aligned}
$$

onde as entradas de $N^{(2)}$ estão em $\mathbf{m}^{k+3}$. Iterando este processo podemos construir uma série formal $\xi_{i}$, e matrizes $A$ e $B$ com entradas no anel das séries formais. Quando $\mathbb{K}=\mathbb{C}$, aplicando o Teorema de Aproximação de Artin, a afirmação se verifica no anel das séries de potências convergentes.

2. $M_{a}+t N \sim M_{a}$, para $t$ suficientemente pequeno. 
Seja $F(x, t)=M_{a}(x)+t N(x)$, pelo primeiro passo feito acima, temos que:

$$
N \in \mathbf{m}^{2}\left(\frac{\partial M_{a}}{\partial x_{1}}, \ldots, \frac{\partial M_{a}}{\partial x_{m}}\right)+\mathbf{m} \operatorname{Im}\left(g_{a}\right) .
$$

Logo,

$$
\frac{\partial F(x, t)}{\partial t}=N(x) \in \mathbf{m}^{2}\left(\frac{\partial M_{a}}{\partial x_{1}}, \ldots, \frac{\partial M_{a}}{\partial x_{m}}\right)+\mathbf{m} \operatorname{Im}\left(g_{a}\right) .
$$

Pelo Lema (2.22), existem $\phi_{1}, \ldots, \phi_{m} \in \mathbb{C}\{x, t\}, A \in \operatorname{Mat}_{(n+1)}(\mathbb{C}\{x, t\}), B \in$ $\mathcal{M a t}_{(n)}(\mathbb{C}\{x, t\})$ tais que

- $A(x, 0)=I d_{n+1}$,

- $B(x, 0)=I d_{n}$,

- $\phi_{i}(x, 0)=x_{i}$,

- $\phi_{i}-x_{i} \in\langle x\rangle^{2}$,

- $A \cdot F(x, t) \cdot B=F\left(\phi_{1}, \ldots, \phi_{m}, 0\right)$.

Daí temos que $A \cdot\left(M_{a}(x)+t N\right) \cdot B=M_{a}\left(\phi_{1}, \ldots, \phi_{m}\right)$, assim $A \cdot\left(M_{a}(x)+t N\right) \cdot B=$ $\phi^{*}\left(M_{a}(x)\right)$. Portanto $M_{a}+t N \sim_{\mathcal{G}} M_{a}$.

3. Provemos que $M \sim_{\mathcal{G}} M+N$.

Nos passos anteriores, vimos que para todo $a \in[0,1]$ existe uma vizinhança $U$ de $a$ tal que para todo $t \in U$, temos $M_{a}+t N \sim M_{a}$. Como o intervalo [0,1] é compacto, podemos cobrí-lo por abertos $U_{i}$. Usando a transitividade da relação de equivalência $\sim$ obtemos

$$
M=M_{0} \sim_{\mathcal{G}} M_{1}=M+N
$$

Apresentamos na seqüência uma outra demonstração do critério infinitesimal de determinação finita que usa o Teorema abc de Hironaka [25], em uma nova versão feita por D. Van Straten e Konrad Möhring em seu trabalho [27]. Antes de mostrar o teorema daremos alguns resultados básicos para a prova.

Proposição 2.24. Seja $P$ um anel Noetheriano, a $\subset P$ um ideal, e $\lambda: A \rightarrow B$ qualquer homomorfismo entre P-módulos finitamente gerados. Então existe $c=c(\lambda) \in \mathbb{N}$ com as seguintes propriedades: $\forall x \in A$ e $p \in \mathbb{N}$ tais que $\lambda(x) \equiv 0 \bmod \mathbf{a}^{p+c} B$ existe um $\tilde{x} \in A$ tal que

$$
\lambda(\tilde{x})=0, e
$$




$$
\tilde{x} \equiv x \bmod \mathbf{a}^{p} A
$$

Demonstração: Como $i m(\lambda)=\lambda(A) \subset B$ é um $P$-submódulo de $B$ temos que $B \supset \mathbf{a} B \supset \mathbf{a}^{2} B \supset \mathbf{a}^{3} B \supset \ldots$ é uma a-filtração a-estável. Então pelo Lema de ArtinRees [14] existe $c$ inteiro tal que

$$
i m(\lambda) \cap \mathbf{a}^{p+c} B=\mathbf{a}^{p}\left(i m(\lambda) \cap \mathbf{a}^{c} . B\right) .
$$

Seja $x \in A$ tal que $\lambda(x) \in \mathbf{a}^{p+c} B$ então $\lambda(x)=\sum_{i} r_{i} n_{i}$, com $r_{i} \in \mathbf{a}^{p}$ e $n_{i}=\lambda\left(m_{i}\right)$ com $m_{i} \in A$. Se $\tilde{x}=x-\sum_{i} r_{i} m_{i}$, podemos verificar que $\lambda(\tilde{x})=0$.

Lema 2.25. Seja $P$ um anel, a $\subset P$ um ideal, $M$ um $P$ - módulo, $A$ um submódulo de $M e$ $\phi \in \operatorname{Aut}_{P}(M)$. Se

$$
A \cap \mathbf{a}^{p+c} M=\mathbf{a}^{p}\left(A \cap \mathbf{a}^{c} M\right)
$$

para algum $p, c \in \mathbb{N}$, então

$$
\phi(A) \cap \mathbf{a}^{p+c} M=\mathbf{a}^{p}\left(\phi(A) \cap \mathbf{a}^{c} M\right) .
$$

Demonstração: Temos que as seguintes propriedades se cumprem para quaisquer $B_{1}$, $B_{2}$ submódulos de $M$

- $\phi\left(B_{1} \cap B_{2}\right)=\phi\left(B_{1}\right) \cap \phi\left(B_{2}\right)$,

- $\phi\left(\mathbf{a}^{p} B_{1}\right)=\mathbf{a}^{p} \phi\left(B_{1}\right)$.

Aplicando $\phi$ a (2.4) obtemos o resultado.

Teorema 2.26. ([27]) Seja $S$ um anel noetheriano comutativo com $1, \mathcal{P}=S\left[\left[x_{1}, \ldots, x_{m}\right]\right] e$ $\mathbf{a} \subset P$ um ideal tal que $1-x$ é invertível $\forall x \in \mathbf{a}$, e a matriz $(I d+\Psi)$ é invertível para toda matriz $\Psi$ com entradas nas potências do ideal a. Seja $I \subset P$ um ideal próprio de $P$ e escreva $\mathcal{R}=\mathcal{P} / I$. Assuma que $\mathbf{a}^{a} T^{1}(\mathcal{R})=0$ para algum $a \in \mathbb{N}$. Para uma seqüência exata de $P$ módulos

$$
\mathfrak{F}: 0 \longrightarrow \mathcal{P}^{r} \stackrel{G}{\longrightarrow} \mathcal{P}^{s} \stackrel{F}{\longrightarrow} \mathcal{P} \longrightarrow \frac{\mathcal{P}}{I} \longrightarrow 0,
$$

existem constantes $a_{F}, a_{G}$ e $b$, tais que o seguinte se verifica: se $c \in \mathbb{N}_{0}, F^{\prime} \equiv F\left(\bmod \mathbf{a}^{a_{F}+c}\right)$, $G^{\prime} \equiv G\left(\bmod \mathbf{a}^{a_{G}}\right)$ e $F^{\prime} \circ G^{\prime}=0$, então existe um automorfismo $\Phi$ de $P$ sobre $S$, congruente à identidade modulo $\mathbf{a}^{a_{F}+c-b}$, que leva o ideal $I^{\prime}=\operatorname{Im}\left(F^{\prime}\right)$ ao ideal $I=\operatorname{Im}(F)$.

Observação 2.27. Consideramos $T^{1}(\mathcal{R})$ como as deformações de primeira ordem de $\left(X_{0}, 0\right)$, onde $X_{0}=V(I)$. Isto é $T^{1}(\mathcal{R})=T_{X_{0}}^{1}$. 
Demonstração: Denotemos $G=\left(G_{i j}\right)$ onde $G_{i j}$ são as entradas da matriz $G$, e $F=$ $\left(f_{1}, \ldots, f_{s}\right)$ onde $f_{i}$ são as entradas da matriz $F$.

Da seqüência exata $(\mathfrak{F})$

$$
\mathfrak{F}: 0 \longrightarrow \mathcal{P}^{r} \stackrel{G}{\longrightarrow} \mathcal{P}^{s} \stackrel{F}{\longrightarrow} I \longrightarrow 0
$$

obtemos o seguinte complexo:

$$
\mathfrak{F}^{*}: 0 \longrightarrow \operatorname{Hom}_{\mathcal{P}}(I, \mathcal{R}) \stackrel{F^{*}}{\longrightarrow} \operatorname{Hom}_{\mathcal{P}}\left(\mathcal{P}^{s}, \mathcal{R}\right) \stackrel{G^{*}}{\longrightarrow} \operatorname{Hom}_{\mathcal{P}}\left(\mathcal{P}^{r}, \mathcal{R}\right)
$$

também temos um mergulho do módulo $N=\operatorname{Hom}_{P}(I, R)$ em $\mathcal{R}^{s}$ :

$$
\begin{aligned}
0 \longrightarrow \operatorname{Hom}_{\mathcal{P}}(I, \mathcal{R}) \stackrel{F^{*}}{\longrightarrow} \operatorname{Hom}_{\mathcal{P}}\left(\mathcal{P}^{s}, \mathcal{R}\right) \stackrel{\cong}{\longrightarrow} \mathcal{R}^{s}, \\
\eta \longmapsto F^{*}(\eta) \longmapsto\left(\eta\left(f_{1}\right), \ldots, \eta\left(f_{s}\right)\right) .
\end{aligned}
$$

As entradas de $F^{\prime}$ e $G^{\prime}$ são:

$$
\begin{gathered}
f_{i}^{\prime}=f_{i}+\phi_{i}, \phi_{i} \in \mathbf{a}^{a_{F}}, \\
g_{i j}^{\prime}=g_{i j}+\gamma_{i j}, \gamma_{i j} \in \mathbf{a}^{a_{G}},
\end{gathered}
$$

com $a_{F}, a_{G} \gg 0$. Depois daremos as cotas inferiores para $a_{F}$ e $a_{G}$ durante a prova. Assumimos que

$$
\begin{aligned}
0 & =F \circ G \\
0 & =\sum_{i=1}^{s} f_{i}^{\prime} g_{i j}^{\prime} \\
0 & =\underbrace{\sum_{i=1}^{s} f_{i} g_{i j}}_{=0}+\sum_{i=1}^{s} \phi_{i} g_{i j}+\underbrace{\sum_{i=1}^{s} f_{i} \gamma_{i j}}_{\in I}+\underbrace{\sum_{i=1}^{s} \phi_{i} \gamma_{i j}}_{\in \mathbf{a}^{a_{F}+a_{G}}} .
\end{aligned}
$$

Assim $\bar{\eta}\left(f_{i}\right)=\left(f_{i}^{\prime}-f_{i}\right)=\phi_{i}$ define um $P$ - homomorfismo de módulos $\bar{\eta}: I \rightarrow \frac{P}{I+\mathbf{a}^{a_{F}+a_{G}}}$ com a propriedade

$$
\bar{\eta}\left(f_{i}\right)=\phi_{i}+\left(I+\mathbf{a}^{a_{F}+a_{G}}\right) .
$$

Queremos encontrar um elemento $\eta \in N=\operatorname{Hom}(I, \mathcal{R})$, que induz $\bar{\eta}$. 
Considere o seguinte homomorfismo:

$$
\begin{aligned}
\lambda: \underbrace{\operatorname{Hom} m_{\mathcal{P}}\left(\mathcal{P}^{s}, \mathcal{P}\right)}_{=A} & \longrightarrow \underbrace{\operatorname{Hom} \mathcal{P}\left(\mathcal{P}^{r}, \mathcal{R}\right)}_{=B} \\
\phi & \longmapsto \phi \circ G \bmod I,
\end{aligned}
$$

onde

$$
\begin{aligned}
\phi \circ G \bmod I: \mathcal{P}^{r} & \longrightarrow c \\
x & \longmapsto \phi \circ G(x)+I .
\end{aligned}
$$

Temos que $\phi=\left(\phi_{1}, \ldots, \phi_{s}\right) \in \operatorname{Hom}_{\mathcal{P}}\left(\mathcal{P}^{s}, P\right)$ então,

$$
\begin{aligned}
\lambda(\phi) & =\phi \circ G \bmod I \\
& =\sum_{i=1}^{s} \phi_{i} g_{i j}+I \\
\lambda(\phi) & \equiv 0 \bmod \mathbf{a}^{a_{F}+a_{G}} .
\end{aligned}
$$

Então pelo Teorema (2.24), existem $c_{1}=c_{1}(\lambda)$ e $\widetilde{\phi} \in A$ tais que $\lambda(\widetilde{\phi})=0$ e $\widetilde{\phi} \equiv$ $\phi \bmod \mathbf{a}^{a_{F}+a_{G}-c_{1}} A$. Quer dizer que:

$$
\begin{aligned}
\widetilde{\phi}_{i} & \equiv \phi_{i} \bmod \mathbf{a}^{a_{F}+a_{G}-c_{1}} \mathrm{e} \\
\sum_{i=1}^{s} \widetilde{\phi}_{i} g_{i j} & =0 \bmod I, \forall j=1, \ldots, r
\end{aligned}
$$

assim este $\widetilde{\phi}_{i}$ descreve um $\eta \in N$ definido por:

$$
\eta\left(f_{i}\right)=\widetilde{\phi}_{i}+I
$$

Assuma que $a_{G}>c_{1}$. Como $\widetilde{\phi}_{i} \equiv \phi_{i} \bmod \mathbf{a}^{a_{F}+a_{G}-c_{1}}$ por 2.8) e pelo fato que $\mathbf{a}^{a_{F}+a_{G}-c_{1}} \subseteq$ $\mathbf{a}^{a_{F}}$, se tem que $\widetilde{\phi}_{i} \equiv \phi_{i} \bmod \mathbf{a}^{a_{F}}$ e desde que $\phi_{i} \in \mathbf{a}^{a_{F}}$ por 2.6) temos que

$$
\widetilde{\phi}_{i} \in \mathbf{a}^{a_{F}} .
$$

Vamos indicar por $\beta$ o mergulho

$$
\begin{aligned}
\beta: N & \longrightarrow \mathcal{R}^{s} \\
\phi & \longmapsto\left(\phi\left(f_{1}\right), \ldots, \phi\left(f_{s}\right)\right) .
\end{aligned}
$$


Temos que $\eta \in N$ (definido em (2.9)) satisfaz.

$$
\begin{aligned}
& \beta(\eta)=\left(\eta\left(f_{1}\right), \ldots, \eta\left(f_{s}\right)\right)=(\underbrace{\widetilde{\phi}_{1}}_{\in \mathbf{a}^{a_{F}}}+I, \ldots, \underbrace{\widetilde{\phi}_{s}}_{\in \mathbf{a}^{a_{F}}}+I) \\
& \beta(\eta) \equiv 0 \bmod \mathbf{a}^{a_{F} \mathcal{R}^{s}} .
\end{aligned}
$$

Pela Proposição (2.24), existem $c_{2}>0$ e $\widetilde{\eta} \in N$ tais que $\beta(\widetilde{\eta})=0$ (i.e $\widetilde{\eta}=0$ ) e $\eta \equiv$ $0 \bmod \mathbf{a}^{a_{F}-c_{2}} N$, i.e $\eta \in \mathbf{a}^{a_{F}-c_{2}} N$.

Queremos encontrar uma derivação $\theta \in \operatorname{Der}_{S}(\mathcal{P}, \mathcal{P})$, cuja restrição a $I$ induz $\eta$. O cokernel de $\xi: \operatorname{Der}_{S}(\mathcal{P}, \mathcal{P}) \rightarrow N$ é por definição, $T^{1}(\mathcal{R} / S)=\frac{N}{i m(\xi)}$. Como $\mathbf{a}^{a} N \subseteq$ $\operatorname{Im}(\xi)$ então $\mathbf{a}^{a_{F}-c_{2}} N \subseteq \mathbf{a}^{a_{F}-c_{2}-a} \operatorname{Im}(\xi)$. Como $\eta \in \mathbf{a}^{a_{F}-c_{2}} N, \eta$ é induzido por algum

$$
\theta \in \mathbf{a}^{a_{F}-a-c_{2}} \operatorname{Der}_{S}(\mathcal{P}, \mathcal{P})
$$

tal que $\xi(\theta)=\eta$, então

$$
\eta\left(f_{i}\right)=\theta\left(f_{i}\right)+I
$$

Por (2.9) e por (2.10) segue que

$$
\theta\left(f_{i}\right) \equiv \phi_{i} \bmod \mathbf{a}^{a_{F}+a_{G}-c_{1}}+I .
$$

Mas, $\theta\left(f_{i}\right)-\widetilde{\phi}_{i} \in I$ o que implica que $\theta\left(f_{i}\right)-\phi_{i} \in I+\mathbf{a}^{a_{F}+a_{G}-c_{1}}$. Por 2.11) e 2.7) sabemos que $\theta\left(f_{i}\right) \in \mathbf{a}^{a_{F}-a-c_{2}} \mathcal{P}$, pois $\phi_{i} \in \mathbf{a}^{a_{F}} \subseteq \mathbf{a}^{a_{F}-a-c_{2}} \mathcal{P}$ então $\theta\left(f_{i}\right)-\phi_{i} \in \mathbf{a}^{a_{F}-a-c_{2}}$ portanto:

$$
\theta\left(f_{i}\right) \equiv \phi_{i} \bmod \mathbf{a}^{a_{F}-a-c_{2}} .
$$

Agora aplicando o Lema de Artin-Rees [14] para $I \subseteq$ a, existe um inteiro $c_{3}$ tal que

$$
\mathbf{a}^{p+c_{3}} \cap I=\mathbf{a}^{p}\left(\mathbf{a}^{c_{3}} \cap I\right) \subseteq \mathbf{a}^{p} I .
$$

Escolhemos $a_{G}>c_{1}$. Assim $a_{F}-a-c_{2}<a_{F}+a_{G}-1$ e (2.15) implica que $\mathbf{a}^{a_{F}-a-c_{2}-c_{3}=c_{3}} \cap$ $\left(I+\mathbf{a}^{a_{F}+a_{G}-c_{1}}\right)=a^{a_{F}-a-c_{2}-c_{3}}\left(I \cap \mathbf{a}^{c_{3}}\right)+\mathbf{a}^{a_{F}+a_{G}-c_{1}} \subset \mathbf{a}^{a_{F}-a-c_{2}-c_{3}} I+\mathbf{a}^{a_{F}=a_{G}-c_{1}}$. Combinando isto com (2.13) e (2.14) obtemos:

$$
\theta\left(f_{i}\right) \equiv \phi_{i} \bmod \mathbf{a}^{a_{F}-a-c_{2}-c_{3}} I+\mathbf{a}^{a_{F}+a_{G}-c_{1}} .
$$


pois,

$$
\begin{aligned}
\theta\left(f_{i}\right) & \equiv \phi_{i} \bmod I+\mathbf{a}^{a_{F}+a_{G}-c_{1}} \\
& \equiv \phi_{i} \bmod \mathbf{a}^{a_{F}-a-c_{2}} \\
\text { então } \theta\left(f_{i}\right) & \equiv \phi_{i} \bmod \left(I+\mathbf{a}^{a_{F}+a_{G}-c_{1}}\right) \cap \mathbf{a}^{a_{F}-a-c_{2}} .
\end{aligned}
$$

Usando a derivação $\theta$ podemos construir um automorfismo $\Phi_{a_{F}}$ de $\mathcal{P}=S\left[\left[x_{1}, \ldots, x_{m}\right]\right]$, pondo

$$
\Phi_{a_{F}}\left(x_{n}\right)=x_{n}-\theta\left(x_{n}\right) .
$$

De (2.11) temos as seguintes inclusões

$$
\begin{aligned}
& \theta\left(\mathbf{a}^{k}\right) \subset \mathbf{a}^{a_{F}-a-c_{2}} \theta\left(\mathbf{a}^{k}\right)=\mathbf{a}^{a_{F}-a-c_{2}+k-1}, \\
& \text { e } \quad \Phi_{a_{F}}(f) \equiv f-\theta(f) \bmod \mathbf{a}^{2\left(a_{F}-a-c_{2}\right)} \quad \forall f \in \mathcal{P} \text {. }
\end{aligned}
$$

Note que

$$
\Phi_{a_{F}} \equiv I d_{\mathcal{P}} \bmod \mathbf{a}^{a_{F}-a-c_{2}}
$$

além disso:

$$
\begin{array}{rlrl}
\Phi_{a_{F}}\left(f_{i}+\phi_{i}\right) & =\Phi_{a_{F}}\left(f_{i}\right)+\Phi_{a_{F}}\left(\phi_{i}\right) & \\
\operatorname{por}(2.18) & & \equiv f_{i}+\left(\phi_{i}-\theta\left(f_{i}\right)\right)-\theta\left(\phi_{i}\right) & \bmod \mathbf{a}^{2\left(a_{F}-a-c_{2}\right)} \\
\operatorname{por}(2.16) & & & \bmod \left(\mathbf{a}^{a_{F}-a-c_{2}-c_{3}} I+\mathbf{a}^{a_{F}+a_{G}-c_{1}}\right) \\
\operatorname{por}(2.17) & & & \bmod \mathbf{a}^{2 a_{F}-a-c_{2}-1} .
\end{array}
$$

Se escolhemos $a_{G} \geq c_{1}+1$ e $a_{F} \geq \max \left\{2 a+2 c_{2}+1, a+c_{2}+2, a_{G}+a+c_{2}+c_{3}\right\}$, obtemos que $a_{F}-a-c_{2}-c_{3} \geq a_{G}$. Então $\mathbf{a}^{a_{F}-a-c_{2}-c_{3}} \subset \mathbf{a}^{a_{G}}$. Além disso $a_{F}+a_{G}-c_{1} \geq a_{F}+1$, portanto $\mathbf{a}^{a_{F}+a_{G}-c_{1}} \subset \mathbf{a}^{a_{F}+1}$. Assim obtemos

$$
\begin{aligned}
\Phi_{a_{F}}\left(f_{i}+\phi_{i}\right) & \equiv f_{i} & \bmod \mathbf{a}^{a_{G}} I+\mathbf{a}^{a_{F}+1} \\
\leftrightarrow \Phi_{a_{F}}\left(f_{i}+\phi_{i}\right) & =f_{i}+\psi_{i}+\phi_{i}^{\prime \prime} & \operatorname{com} \psi_{i} \in \mathbf{a}^{a_{G}} I, \phi_{i}^{\prime \prime} \in \mathbf{a}^{a_{F}+1} .
\end{aligned}
$$

Considere o vetor $\left(f_{1}+\psi_{i}, \ldots, f_{s}+\psi_{s}\right)$, que pode ser escrito como o seguinte produto $\left(f_{1}, \ldots, f_{s}\right) \circ\left(I d_{s}+\Psi_{a_{F}}\right)$, onde $\Psi_{a_{F}}$ é uma matriz com entradas em $\mathbf{a}^{a_{G}}$. A matriz $\left(I d_{S}+\right.$ $\left.\Psi_{a_{F}}\right)$ é invertível, então descreve um automorfismo em $\mathcal{P}^{s}$. Seja $\widetilde{F}=F \circ\left(I d_{s}+\Psi_{a_{F}}\right)$ e 
$\widetilde{G}=\left(I d_{S}+\Psi_{a_{F}}\right)^{-1} \circ G$. Obtemos uma nova representação de $\mathcal{R}$ :

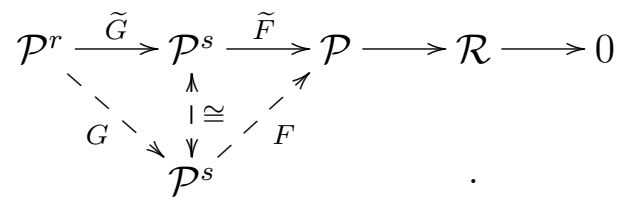

Agora Seja $G^{\prime \prime}=G^{\prime}$ e $F^{\prime \prime}=\Phi_{a_{F}} \circ F^{\prime}$ :

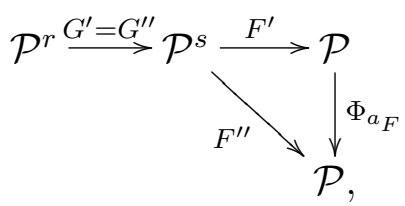

então $F^{\prime \prime} \circ G^{\prime \prime}=0$. Temos que as entradas de $\widetilde{F}$ são congruentes às entradas de $F^{\prime \prime}$ módulo $\mathbf{a}^{a_{F}+1}$ pois as entradas da matriz $F \circ\left(I d_{s}+\Psi_{a_{F}}\right)-\Phi_{a_{F}} \circ F^{\prime}$, são da forma $f_{i}+\psi_{i}-f_{i}-\psi_{i}-\phi^{\prime \prime} \in \mathbf{a}^{a_{F}+1}$. Além disso as entradas de $\widetilde{G}$ são congruentes às entradas de $G$ módulo a ${ }^{a_{G}}$, e $G \equiv G^{\prime \prime}\left(=G^{\prime}\right) \bmod \mathbf{a}^{a_{G}}$ portanto $\widetilde{G} \equiv G^{\prime \prime} \bmod \mathbf{a}^{a_{G}}$.

Agora queremos usar indução sobre $a_{F}$, e para isso verifiquemos se todas as constantes podem ser tomadas para o próximo passo de indução.

As constantes $a$ e $c_{3}$ são obtidas diretamente dos ideais a e $I$.

A constante $c_{1}$ é obtida pela Proposição (2.24) aplicando o homomorfismo

$$
\begin{aligned}
\lambda: \underbrace{\operatorname{Hom}}_{=A}\left(\mathcal{P}^{s}, \mathcal{P}\right) & \longrightarrow \underbrace{\operatorname{Hom}\left(\mathcal{P}\left(\mathcal{P}^{r}, \mathcal{R}\right)\right.}_{=B} \\
\phi & \longmapsto \phi \circ G \bmod I .
\end{aligned}
$$

Para o próximo passo da indução aplicamos o homomorfismo

$$
\begin{aligned}
\lambda^{\prime}: A & \longrightarrow B \\
\phi & \longmapsto \phi \circ\left(I d_{s}+\Psi_{a_{F}}\right)^{-1} \circ G \bmod I .
\end{aligned}
$$

Como o inteiro $c_{1}$ só depende da imagem de $\lambda$ e como $\left(\left(I d_{s}+\Psi_{a_{F}}\right)\right.$ é um automorfismo, então a constante é a mesma.

A constante $c_{2}$ surge do mergulho $\beta$. Isto foi encontrado aplicando o Lema de Artin Rees ao submódulo $F^{*}(\operatorname{Hom}(I, \mathcal{R})) \subset \operatorname{Hom}_{\mathcal{P}}\left(\mathcal{P}^{s}, \mathcal{R}\right) \cong \mathcal{R}^{s}$. No próximo passo consideramos o seguinte submódulo $\left(\left(I d_{s}+\Psi_{a_{F}}\right)^{*}\left(F^{*}(\operatorname{Hom}(I, \mathcal{R}))\right) \subset H_{\mathcal{P}}\left(\mathcal{P}^{s}, \mathcal{R}\right)\right.$, e como $\left(I d_{s}+\Psi_{a_{F}}\right)^{*}$ é um $\mathcal{P}$ automorfismo de $\operatorname{Hom}_{\mathcal{P}}\left(\mathcal{P}^{s}, \mathcal{R}\right)$, aplicamos o Lema 2.25). Assim a constante $c_{2}$ é a mesma.

Agora para a indução, $\Phi_{a_{F}} \equiv \operatorname{Id} \bmod \mathbf{a}^{a_{F}-a-c_{2}}$, considere $\Phi=\ldots \circ \Phi_{a_{F}+1} \circ \Phi_{a_{F}}$ que 
é um isomorfismo de $\mathcal{P}$. Do mesmo modo, obtemos uma matriz $\Psi$ com entradas em alguma potência de a tal que $(1+\Psi)=\prod\left(1+\Psi_{a_{F}+k}\right)$. Por construção, $\Phi\left(f_{i}+\phi_{i}\right)$ é a i-ésima componente de $\left(f_{1}, \ldots, f_{s}\right) \circ(I d+\Psi)$. Assim $\left\{f_{i}+\phi_{i}\right\}$ está sendo aplicado ao sistema de geradores $\left\{f_{i} \circ(I d+\Psi)\right\}$ de $I, \log 0 \Phi\left(I^{\prime}\right)=I$.

Corolário 2.28. ([27]) Seja $\mathbb{K}$ um corpo, $\mathcal{P}=\mathbb{K}\left[\left[x_{1}, \ldots, x_{n}\right]\right], \mathbf{m}=\left(x_{1}, \ldots, x_{n}\right), I=$ $\left(f_{1}, \ldots, f_{k}\right) \subset \mathcal{P}$ um ideal e $\mathcal{R}=\mathcal{P} /$ I. Seja:

$$
\mathfrak{F}: 0 \longrightarrow \mathcal{P}^{l} \stackrel{G}{\longrightarrow} \mathcal{P}^{k} \stackrel{F}{\longrightarrow} \mathcal{P} \longrightarrow \frac{\mathcal{P}}{I} \longrightarrow 0
$$

uma resolução livre de $\mathcal{R}, F=\left(f_{1}, \ldots, f_{k}\right) \in \operatorname{Hom}\left(\mathcal{P}^{k}, \mathcal{P}\right)$ e $G \in \operatorname{Hom}\left(\mathcal{P}^{l}, \mathcal{P}^{k}\right)$. Se além $\operatorname{disso}_{\operatorname{dim}_{k}} T_{\mathcal{R}}^{1}<\infty$, então existem inteiros naturais a, $b, c$, tais que o seguinte é verdadeiro: $\forall$ $p \geq$ c e para todo $F^{\prime}=\left(f_{1}^{\prime}, \ldots, f_{n}^{\prime}\right) \in \operatorname{Hom}\left(\mathcal{P}^{k}, \mathcal{P}\right)$ e $G^{\prime} \in H o m\left(\mathcal{P}^{l}, \mathcal{P}^{k}\right)$ com as seguintes propriedades

1. $F^{\prime} \circ G^{\prime}=0$

2. $F^{\prime} \equiv F\left(\bmod \mathbf{m}^{a+p}\right)$

3. $G^{\prime} \equiv G\left(\bmod \mathbf{m}^{b}\right)$

existe um isomorfismo $\Phi: \mathbb{K}\left[\left[x_{1}, \ldots, x_{m}\right]\right] \rightarrow \mathbb{K}\left[\left[x_{1}, \ldots, x_{m}\right]\right]$ tal que $\Phi\left(f_{i}^{\prime}\right)=f_{i} \forall 1 \leq i \leq k$ e $\Phi \equiv \operatorname{Id}\left(\bmod \mathbf{m}^{p}\right)$

Demonstração: Segue diretamente do Teorema anterior considerando $S=\mathbb{K}, \mathbf{a}=\mathbf{m}$. A condição que $\operatorname{dim}_{k} T_{\mathcal{R}}^{1}<\infty$ implica que $\exists a \in \mathbb{N}$ tal que $\mathbf{m}^{a} \mathcal{M} a t_{(n+1) n}(\{x\}) \subset J(M)+$ $\operatorname{Im}(g)$ o que significa que $\mathbf{m}^{a} T^{1}(M)=0$.

O seguinte resultado é o critério infinitesimal para a determinação finita que resulta como uma aplicação de teorema acima mencionado.

Corolário 2.29. Seja $M \in \mathcal{M a t}(\mathbb{C}\{x\})$ com entradas no ideal maximal de $\mathbb{C}\{x\}$ de codimensão finita, isto é, $\operatorname{dim}_{\mathbb{C}} T^{1}(M)<\infty$. Então $M$ é finitamente determinado, i.e. existe $r \in \mathbb{N}$, tal que $M \sim_{\mathcal{G}} \widetilde{M}$ para alguma matriz $\widetilde{M} \in \mathcal{M}$ at $(\mathbb{C}\{x\})$ que satisfaz a condição $M_{i j}-\widetilde{M}_{i j} \in \mathbf{m}^{r}$.

Demonstração: Segue diretamente do Corolário (2.28) e da Proposição (2.1) e do fato que $\operatorname{dim}_{\mathbb{C}} T^{1}(M)<\infty$.

Observação 2.30. É possível demonstrar que o grupo $\mathcal{G}(n)$ é um subgrupo geométrico do grupo do contato $\mathcal{K}$, definido por J. Damon e como conseqüiência dos resultados de [11], podemos usar as técnicas da teoria de singularidades (por exemplo, os resultados com respeito à determinação finita e deformações) para investigar estas singularidades. Estes fatos podem ser encontrados na tese de Miriam da Silva Pereira [10]. 


\subsection{Matrizes Quase- Homogêneas}

Definição 2.31. Seja $g\left(x_{1}, \ldots, x_{m}\right)=x_{1}^{v_{1}} \ldots x_{m}^{v_{m}}$ um monômio. Definamos o grau de $g$ com respeito aos pesos $\underline{a}=\left(a_{1}, \ldots, a_{m}\right) ; 0<a_{i} \in \mathbb{N}, \forall 1 \leq i \leq m$, por

$$
v_{a}(g)=\sum_{i=1}^{m} a_{i} v_{i} .
$$

Seja $f$ um polinômio. Definamos a ordem de $f$ com relação aos pesos $\underline{a}=\left(a_{1}, \ldots a_{m}\right)$ por

$$
v_{a}(f)=\inf \left\{v_{a}(g) / g \text { é um monômio def }\right\} \text {. }
$$

Dizemos que $f$ é quase-homogêneo de grau d com respeito aos pesos $\underline{a}$, se $v_{a}(g)=d$ para qualquer monômio $g$ de $f$.

Podemos considerar $\mathcal{P}$ como um módulo graduado associado a um sistema de pesos.

$$
\mathcal{P}=\bigoplus_{d \geq 0} G_{d}^{a}
$$

onde $G_{d}^{a}$ são todos os polinômios quase-homogêneos de tipo $(a, d)$. Por convenção consideramos $G_{d}^{a}=0$ se $d<0$.

Observação 2.32. Um polinômio quase-homogêneo de tipo $(a, d)$ satisfaz a relação de Euler, ${ }^{1}$

$$
d . f=\sum_{i=1}^{n} a_{i} x_{i} \frac{\partial f}{\partial x_{i}} .
$$

Definição 2.33. Seja $M \in \operatorname{Mat}_{(n+1) n}\left(\mathbb{C}\left\{x_{1}, \ldots, x_{m}\right\}\right)$. $M$ é chamado quase homogêneo de tipo $(D, a) \in \mathcal{M a t}_{(n+1) n}(\mathbb{N}) \times \mathbb{N}^{m}$, se:

a) Todas as entradas $M_{i j}$ são quase-homogêneas de grau $D_{i j}$ com respeito a $a$.

b) $D_{i j}-D_{i k}=D_{l j}-D_{l k}$ para $1 \leq i, l \leq n+1,1 \leq j, k \leq n$.

Seja $N$ outra matriz com entradas em $\mathcal{P}$. O peso relativo da matriz com respeito a $(D, a)$ é dado por

$$
v_{(D, a)}(N)=\inf _{j, i}\left\{v_{a}\left(N_{i j}\right)-D_{i j}\right\}
$$

\footnotetext{
${ }^{1}$ A relação de Euler generaliza a formula de Euler para polinômios homogêneos.
} 
De novo, sobre esta definição podemos considerar o espaço das matrizes $(n+1) \times n$, $\operatorname{Mat}_{(n+1) n}(\mathcal{P})$, como um módulo graduado:

$$
\operatorname{Mat}_{(n+1) n}(\mathcal{P})=\bigoplus_{v \in \mathbb{Z}} G_{v}^{D ; a}
$$

onde $G_{v}^{D ; a}=\left\{\left(M_{i j}\right) \in \mathcal{M a t}_{(n+1) n}(\mathcal{P}) / M_{i j} \in G_{D_{i j}+v}^{a}\right\}$.

Lema 2.34. Seja I $\subset \mathcal{P}$ um ideal Cohen-Macaulay de codimensão 2, gerado por polinômios quase-homogêneos com relação a algum peso a. Então existe uma representação matricial $M$ de $I$, quase homogênea de tipo $(D, \underline{a})$, para algum $D \in \operatorname{Mat}_{(n+1) n}(\mathbb{N})$.

Demonstração: Suponha que o ideal $I=\left\langle f_{1}, \ldots, f_{n+1}\right\rangle$ seja gerado por $n+1$ elementos. Pela Proposição (2.1) existe uma seqüência exata de $\frac{\mathcal{P}}{I}$. Seja $M \in \operatorname{Mat}_{(n+1) n}(\mathcal{P})$ dada pela seqüência exata, onde $I=a\left(\Delta_{1}, \ldots, \Delta_{n+1}\right)$, com $a$ uma unidade de $\mathcal{P}$. Então $\forall$ $1 \leq i \leq n+1$,

$$
\Delta_{i}=\sum_{i=1}^{n+1} R_{i} f_{i}
$$

é quase-homogênea. Suponha que todas as entradas de $M$ são quase-homogêneas. Então verificando as condições de linhas e colunas segue o resultado.

Por exemplo, para $n=2$, temos pela Proposição (2.1) uma seqüência exata. Seja $M=\left(M_{i j}\right)$ de tamanho $3 \times 2$ dada pela seqüência exata, onde $I=a\left(\Delta_{1}, \Delta_{2}, \Delta_{3}\right)$ e $a$ é uma unidade de $\mathcal{P}$.

Então temos que $\Delta_{1}=M_{21} M_{32}-M_{22} M_{31}, \Delta_{2}=M_{11} M_{32}-M_{12} M_{31}, \Delta_{3}=M_{11} M_{22}-$ $M_{12} M_{21}$ são polinômios quase-homogêneos com respeito aos pesos $a=\left(a_{1}, \ldots, a_{m}\right)$. Pela definição de polinômios quase-homogêneos temos que: $v_{a}\left(M_{21} M_{32}\right)=v_{a}\left(M_{22} M_{31}\right)$, $v_{a}\left(M_{11} M_{32}\right)=v_{a}\left(M_{12} M_{31}\right), v_{a}\left(M_{11} M_{22}\right)=v_{a}\left(M_{12} M_{21}\right)$

o que é equivalente a: $v_{a}\left(M_{21}\right)+v_{a}\left(M_{32}\right)=v_{a}\left(M_{22}\right)+v_{a}\left(M_{31}\right), v_{a}\left(M_{11}\right)+v_{a}\left(M_{32}\right)=$ $v_{a}\left(M_{12}\right)+v_{a}\left(M_{31}\right), v_{a}\left(M_{11}\right)+v_{a}\left(M_{22}\right)=v_{a}\left(M_{12}\right)+v_{a}\left(M_{21}\right)$

O seguinte resultado e sua prova são uma generalização do resultado obtido por Giusti em seu trabalho, [20], para singularidades de intersecções completas.

Corolário 2.35. Seja $M \in \mathcal{M a t}_{(n+1) n}(\mathbb{C}\{x\})$ com entradas no ideal maximal de $\mathbb{C}\{x\}$ quasehomogênea de tipo $(D, a)$ e definindo uma singularidade cujo número de Tjurina é finito. Seja $N \in \operatorname{Mat}_{(n+1) n}(\mathbb{C}\{x\})$ tal que

$$
v_{(D ; a)}(N)>\beta=\sup \{0, \alpha\}
$$


onde $\alpha=\sup \left\{v \in \mathbb{Z} / T_{v}^{1}(M) \neq 0\right\}$.

Então $M+N \sim_{\mathcal{G}} M$.

Demonstração: Sem perda de generalidade podemos assumir que $M$ é ordenado pelos pesos das linhas e colunas, i.e $D_{i j} \leq D_{i k}, \forall j \leq k, D_{i j} \leq D_{k j}, \forall i \leq k$. Como $v_{(D, a)}(N)>B>\alpha$, implica que:

$$
v_{a}\left(N_{i j}\right)-D_{i j}>\inf \left\{v_{a}\left(N_{i j}\right)-D_{i j}\right\}>\alpha
$$

Assim a imagem de $N$ em $T^{1}(M)$ é zero, quer dizer que $N \in J(M)+\operatorname{Im}(g)$,

$$
N=\sum_{i=1}^{m} \xi_{i} \frac{\partial M}{\partial x_{i}}+L M+M R,
$$

onde $\xi \in \mathbb{C}\{x\}, L \in \mathcal{M a t}_{n+1}(\mathbb{C}\{x\})$ e $R \in \mathcal{M a t}_{n}(\mathbb{C}\{x\})$.

Como $v_{(D, a)}(N)>\beta>0$, podemos escolher $v_{a}\left(\xi_{i}\right)>a_{i}, v_{a}\left(L_{i k}\right) \geq v_{a}\left(N_{i j}\right)-D_{i j} \mathrm{e}$ $v_{a}\left(R_{k j}\right) \geq v_{a}\left(N_{i j}\right)-D_{i k}$, isto se deduz da seguinte formula

$$
N_{i j}=\sum_{k=1}^{n} \xi_{k} \frac{\partial M}{\partial x_{k}}+\sum_{k=1}^{n+1} L_{i k} M_{k j}+\sum_{k=1}^{n} M_{i k} R_{k j}
$$

então teríamos que:

$$
\begin{array}{rlc}
v_{a}\left(L_{i k} N_{k j}\right) & \geq & v_{a}\left(L_{i k}\right)+v_{a}\left(N_{k j}\right) \\
& \geq & v_{a}\left(N_{i j}\right)-D_{k j}+v_{a}\left(N_{k j}\right) \\
& \geq & v_{a}\left(N_{i j}\right)+v_{(D, a)}(N) \\
& > & v_{a}\left(N_{i j}\right) . \\
v_{a}\left(N_{i k} R_{k j}\right) & \geq & \\
& \geq & v_{a}\left(N_{i k}\right)+v_{a}\left(R_{k j}\right) \\
& \geq & v_{(D, a)}(N)+v_{a}\left(N_{i j}\right)-D_{i k}\left(N_{i j}\right) \\
& > & \\
& & v_{a}\left(N_{i j}\right) .
\end{array}
$$

Além disso:

$$
\begin{aligned}
& v_{a}\left((L N)_{i j}\right)=v_{a}\left(\sum_{k=1}^{n+1} L_{i k} N_{k j}\right)>v_{a}\left(N_{i j}\right) \text { e assim } \\
& v_{(D, a)}(L N)>v_{(D, a)}(N)
\end{aligned}
$$




$$
\begin{aligned}
& v_{a}\left((N R)_{i j}\right)=v_{a}\left(\sum_{k=1}^{n} N_{i k} R_{k j}\right)>v_{a}\left(N_{i j}\right) \text { e assim } \\
& v_{(D, a)}(N R)>v_{(D, a)}(N) .
\end{aligned}
$$

As condições sobre $v_{a}\left(L_{i k}\right)$ e $v_{a}\left(R_{k j}\right)$ e o fato de $M$ ser ordenada pelos pesos da suas linhas e colunas asseguram que $\left(I d_{n+1}-L\right)(0)$ e $\left(I d_{n}-R\right)(0)$ são matrizes triangulares. Além disso, as entradas da diagonal são 1. Definamos o seguinte elemento em $\mathcal{R}_{m}$

$$
\begin{array}{rlc}
\phi: \mathbb{C}\{x\} & \longrightarrow \mathbb{C}\{x\} \\
x & \longmapsto\left(x_{1}+\xi_{1}, \ldots, x_{m}+\xi_{m}\right) .
\end{array}
$$

Logo,

$$
\begin{aligned}
\phi^{*}(M+N)= & M(\phi(x))+\sum_{i=1}^{m} \xi_{i}(\phi(x)) \frac{\partial M(\phi(x))}{\partial \phi_{i}}+(L M)(\phi(x))+M R(\phi(x)) \\
= & M+\widehat{M}+\sum_{k=1}^{m} \xi_{i} \frac{\partial M}{\partial x_{i}}-\sum_{k=1}^{m} \xi_{i} \frac{\partial M}{\partial x_{i}}+\sum_{i=1}^{m} \xi_{i}(\phi(x)) \frac{\partial M(\phi(x))}{\partial \phi_{i}}+L M+M R \\
& +\widehat{L M}+\widehat{M R} \\
= & M+N-\sum_{i=1}^{m} \xi_{i} \frac{\partial M}{\partial x_{i}}+\underbrace{\widehat{M}+\sum_{i=1}^{m} \xi_{i}(\phi(x)) \frac{\partial M(\phi(x))}{\partial+\phi_{i}}+\widehat{L M}+\widehat{M R}}_{H_{1}}
\end{aligned}
$$

onde $v_{(D, a)}\left(H_{1}\right)>v_{(D, a)}(N)$ então teríamos.

$$
\begin{gathered}
\left(I d_{n+1}-L\right)\left(\phi^{*}(M+N)\right)\left(I d_{n}-R\right) \\
=\quad\left(I d_{n+1}-L\right)\left(M+N-\sum_{k=1}^{m} \xi_{i} \frac{\partial M}{\partial x_{i}}+H_{1}\right)\left(I d_{n}-R\right) \\
=(M+N-\sum_{k=1}^{m} \xi_{i} \frac{\partial M}{\partial x_{i}}-L M-\underbrace{\left.L N+\sum_{k=1}^{m} \xi_{i} L \frac{\partial M}{\partial x_{i}}+H_{1}-L H_{1}\right)}_{=H_{2} ; v_{(D, a)}\left(H_{2}\right)>v_{(D, a)}(N)}\left(I d_{n}-R\right) \\
=\underbrace{M+N-\sum_{k=1}^{m} \xi_{i} \frac{\partial M}{\partial x_{i}}-L M-M R}_{=-N} \underbrace{-N R+\sum_{k=1}^{m} \xi_{i} \frac{\partial M}{\partial x_{i}} R+L M R+H_{2}-H_{2} R}_{M+N_{1} .}
\end{gathered}
$$

Portanto $M+N \sim_{\mathcal{G}} M+N_{1}$.

Como $v_{(D, a)}\left(N_{1}\right)>v_{(D, a)}(N)>\beta$, então fazendo o mesmo processo, encontramos uma 
matriz $N_{2} \in \operatorname{Mat}_{(n+1) n}(\mathbb{C}\{x\})$ tal que $M+N_{1} \sim M+N_{2} \operatorname{com} v_{(D, a)}\left(N_{2}\right)>v_{(D, a)}\left(N_{1}\right)>$ $\beta$. Iterando de novo o processo, então teríamos que existem matrizes $N_{1}, N_{2}, \ldots$ tais que $M+N \sim_{\mathcal{G}} M+N_{k}, \forall k$ e $v_{(D, a)}\left(N_{k+1}\right)>v_{(D, a)}\left(N_{k}\right)$. Temos por hipótese que $M$ define uma singularidade tal que $\operatorname{dim} T^{1}<\infty$ e então pelo Corolário de Hironaka, $M$ é finitamente determinada. Então existe $n$ tal que se $N_{i j} \in \mathbf{m}^{n}$ então $M \sim_{\mathcal{G}} M+N_{n}$, ou seja, $M+N \sim_{\mathcal{G}} M+N_{n} \sim_{\mathcal{G}} M$. 


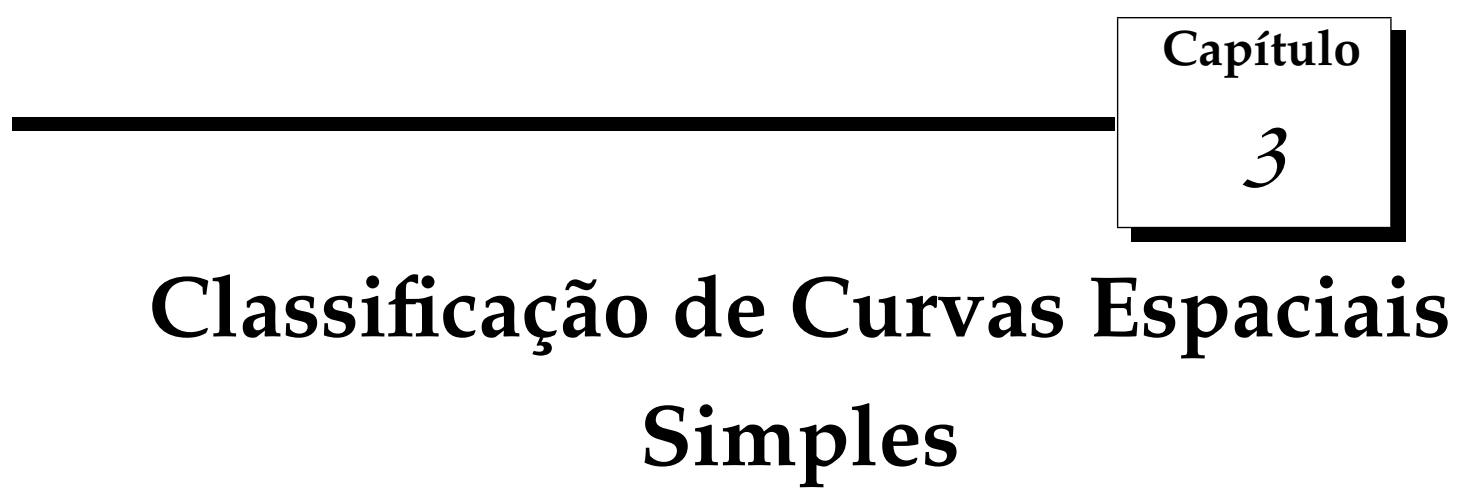

O objetivo deste capítulo é descrever a classificação das curvas determinantais simples, obtida por Anne Frühbis-Krüger em [15].

\subsection{Redução ao Problema}

Vamos introduzir as notações que serão utilizadas para descrever singularidades que não podem ser simples. Antes daremos a seguinte notação. Fixemos os pesos $a=\left(a_{1}, \ldots, a_{m}\right)$. Seja $\left\{b_{1, d}, b_{2, d} \ldots, b_{r(a, d), d}\right\}$ o conjunto de monômios de grau $d$ e denotemos por $r(a, d)$ o número de elementos deste conjunto. Seja $G_{0}$ o $\mathbb{C}$ - espaço vetorial de todas as matrizes quase-homogêneas de tipo $(\mathrm{D}, \mathrm{a})$. Sua base é de cardinalidade $\sum_{i j} r\left(a, D_{i j}\right)$. No problema de classificação que estamos estudando, uma matriz $M \in G_{0}$ será chamada genérica se $M$ é $G(n)$ - finitamente determinada.

Para $M \in G_{0}$ genérica, consideramos os seguintes subconjuntos de $G_{0}$ com suas cardinalidades:

1. $S_{1}=\left\{b_{i, a_{j}} \frac{\partial M}{\partial x_{j}} / 1 \leq i \leq r\left(a, a_{j}\right), 1 \leq j \leq m\right\}, \operatorname{card}\left(S_{1}\right)=s_{1}$,

2. $S_{2}=\left\{b_{i, D_{l 1}-D_{j 1}} Z_{j l} / 1 \leq i \leq r\left(a, D_{l 1}-D_{j 1}\right), 1 \leq j, l \leq n+1\right\}, \operatorname{card}\left(S_{2}\right)=s_{2}$,

3. $S_{3}=\left\{b_{i, D_{1 l}-D_{j l}} S_{j l} / 1 \leq i \leq r\left(a, D_{1 l}-D_{1 j}\right), 1 \leq j, l \leq n\right\}, \operatorname{card}\left(S_{3}\right)=s_{3}$,

onde $Z_{j l} \in \operatorname{Mat}_{(n+1) n}(\mathbb{C}\{x\})$ é a matriz que tem como j-ésima linha a l-ésima linha de $M$ e zeros nas outras entradas, e do mesmo modo $S_{j l} \in \mathcal{M a t}_{(n+1) n}(\mathbb{C}\{x\})$ é a matriz 
que tem como j-ésima coluna a l-ésima coluna de $M$ e zeros nas outras entradas. Estas matrizes podem ser escritas da seguinte forma:

$$
Z_{j l}=\left(\begin{array}{ccc} 
& 0 & \\
M_{j 1} & \ldots & M_{j n} \\
& 0 &
\end{array}\right) \text { e } S_{j l}=\left(\begin{array}{ccc} 
& M_{1 j} & \\
0 & \vdots & 0 \\
& M_{(n+1) j} &
\end{array}\right) .
$$

Os elementos dos três conjuntos $S_{i}$ geram o espaço tangente da órbita de $M$ em $G_{0}$. Ou seja, estes elementos geram o núcleo da aplicação: $G_{0} \rightarrow T^{1}(M)$, que aplica um elemento $M \in G_{0}$ a sua classe em $T^{1}(M)$.

A dimensão deste núcleo é estritamente menor do que: $s_{1}+s_{2}+s_{3}-1$, que é equivalente a dizer que a dimensão do espaço tangente da órbita em $M$ é estritamente menor do que $s_{1}+s_{2}+s_{3}-1$, pois existem ao menos duas relações entre os elementos destes conjuntos:

1. $\sum_{i} Z_{i i}=\sum_{i} S_{i i}$

2. $\sum_{i=1}^{m} a_{i} x_{i} \frac{\partial M}{\partial x_{i}}=\sum_{i=1}^{n} D_{1 i} S_{i i}+\sum_{i=2}^{n+1}\left(D_{i 1}-D_{11}\right) Z_{i i}$, pela equação de Euler (Observação 2.32.

A primeira igualdade segue do fato que $M=\sum_{i} Z_{i i}=\sum_{i} S_{i i}$ e a segunda relação segue da fórmula de Euler para polinômios quase-homogêneos:

$$
\left.\begin{array}{rl}
\sum_{i=1}^{m} a_{i} x_{i} \frac{\partial M}{\partial x_{i}}= & \left(\begin{array}{ccc}
D_{11} M_{11} & \cdots & D_{1 n} M_{1 n} \\
\vdots & \ddots & \vdots \\
D_{(n+1) 1} M_{(n+1) 1} & \cdots & D_{(n+1) n} M_{(n+1) n}
\end{array}\right) \\
= & \left(\begin{array}{ccc}
D_{11} M_{11} & \ldots & D_{1 n} M_{1 n} \\
\vdots & \ddots & \vdots \\
D_{11} M_{(n+1) 1} & \ldots & D_{1 n} M_{(n+1) n}
\end{array}\right)+ \\
0 & \ldots \\
\vdots & \ddots
\end{array}\right)
$$


Pois $D_{(j+1) 1}-D_{11}=\ldots=D_{(j+1) j}-D_{1 j}$, pela relação entre linhas e colunas dada na parte 2 da Definição (2.33).

\subsection{Germes Simples}

Como estamos interessados na classificação de curvas espaciais simples, definiremos germes simples e apresentaremos critérios que os identifiquem. Vamos mostrar também que os possíveis valores para $n$ são 1 e 2 .

Definição 3.1. Uma matriz finitamente determinada $M \in \mathcal{M a t}_{(n+1) n}(\mathcal{P})$ é $\mathcal{G}(n)$ simples se existe um número inteiro $k_{0} \in \mathbb{N}$ e uma vizinhança $V$ de $j^{k} M$ em $J^{k} \mathcal{M a t}_{(n+1) n}(\mathcal{P})$ tais que $V$ encontra um número finito de $J^{k} \mathcal{G}(n)$ - órbitas, para todo $k \geq k_{0}$.

A seguinte proposição nos fornece um critério para detectarmos a existência de germes não simples.

Proposição 3.2. (J. W. Bruce, [? ]) Seja $V$ um $\mathbb{K}$ - espaço afim e $G$ um grupo algébrico de Lie agindo em $V$. Se $W$ é uma subvariedade suave de $V$ com a propriedade que o conjunto $\left\{w \in W / T_{w} W \subset T_{w} G . w\right\}$ é uma subvariedade própria de $W$, então dado qualquer $w \in W$, para toda vizinhança $U$ de $w$ temos que ela encontra um número infinito de $G$-órbitas.

O seguinte lema é uma aplicação da proposição anterior.

Lema 3.3. O espaço de curvas singulares definidas por uma matriz genérica $M \in G_{0}$ de tipo $(D, a)$ não pode ser simples, se $\sum_{i j} r\left(a, D_{i j}\right) \geq s_{1}+s_{2}+s_{3}-1$.

Demonstração: Pelos cálculos da seção anterior temos que $\operatorname{dim}_{M} \cdot \mathcal{G}(n) \cdot M<s_{1}+s_{2}+$ $s_{3}-1$, para $M \in G_{0}$. Se $s_{1}+s_{2}+s_{3}-1 \leq \sum_{i j} r\left(a, D_{i j}\right)$, então teríamos que $\operatorname{dim} T_{M} \cdot \mathcal{G} \cdot M<$ $\operatorname{dim} G_{0}$. Logo, pela Proposição (3.2), uma vizinhança de $M$ em $G_{0}$ não pode ser coberta por um número finito de órbitas.

Corolário 3.4. Seja $M \in \mathcal{M a t}_{(n+1) n}\left(\mathbb{C}\left\{x_{1}, x_{2}, x_{3}\right\}\right)$ com entradas no ideal maximal de $\mathbb{C}\left\{x_{1}, x_{2}, x_{3}\right\}$. Se $n \geq 3$, então $M$ não define um curva simples.

Demonstração: Seja $N$ uma matriz genérica de tipo $(D, a), \operatorname{com} a=(1,1,1), D_{i j}=1 \forall$ $1 \leq i \leq n+1, \forall 1 \leq j \leq n$.

Aplicando o critério do Lema (3.3) temos que calcular a dimensão de $G_{0}$ e obtemos que $r\left(a, D_{i j}\right)=3, \forall i, j$. Então $\sum_{i j} r\left(a, D_{i j}\right)=3 n(n+1)=3 n^{2}+3 n$.

Como $r\left(a, a_{j}\right)=3, \forall 1 \leq j \leq 3$, pois os únicos polinômios mônicos de grau 1 são: 
$x_{1}, x_{2}, x_{3}$ e portanto $s_{1}=3.3=9, s_{2}=(n+1)^{2}$ e $s_{3}=n^{2}$. Assim $N$ e $j^{1}(M)$ não podem ser simples, se:

$$
\sum_{i j} r\left(a, D_{i j}\right)=m . n^{2}+m . n \geq(n+1)^{2}+n^{2}+m^{2}-1=s_{1}+s_{2}+s_{3}-1 \forall n \geq 3 .
$$

A equação 3.1) é satisfeita quando $n \geq 3$. Se o germe definido por $M$ for simples então existe uma vizinhança de $j^{1}(M)$ em $G_{0}$, que encontra um número finito de órbitas, contradizendo o fato que $j^{1}(M)$ não é simples.

Corolário 3.5. Candidatos para curvas espaciais simples podem ser encontrados entre as intersecções completas classificadas por M. Giusti, [20], e entre os germes definidos pelos menores maximais de uma matriz $3 \times 2$.

Vamos mencionar o espaço de curvas classificadas por Giusti em seu trabalho, [20].

$$
\begin{aligned}
& \text { Tipo } S_{u}\left(x^{2}+y^{2}+z^{u-3}, y z\right) \text {, } \\
& \text { Tipos } \begin{cases}T_{7} & \left(x^{2}+y^{3}+z^{3}, y z\right), \\
T_{8} & \left(x^{2}+y^{3}+z^{4}, y z\right), \\
T_{9} & \left(x^{2}+y^{3}+z^{5}, y z\right),\end{cases} \\
& \text { Tipos } \begin{cases}U_{7} & \left(x^{2}+y z, x y+z^{3}\right), \\
U_{8} & \left(x^{2}+y z+z^{3}, x y\right), \\
U_{9} & \left(x^{2}+y z, x y+z^{4}\right),\end{cases} \\
& \text { Tipos } \begin{cases}W_{8} & \left(x^{2}+z^{3}, y^{2}+x z\right), \\
W_{9} & \left(x^{2}+y z^{2}, y^{2}+x z\right),\end{cases} \\
& \text { Tipos } \begin{cases}Z_{9} & \left(x^{2}+z^{3}, y^{2}+z^{3}\right), \\
Z_{10} & \left(x^{2}+y z^{2}, y^{2}+z^{3}\right) .\end{cases}
\end{aligned}
$$

Estas mesmas considerações que usamos para obter o resultado acima também permitem desigualdades similares para dimensões mais altas:

$$
\sum_{i j} r\left(a, D_{i j}\right)=m \cdot n^{2}+m \cdot n \geq(n+1)^{2}+n^{2}+m^{2}-1
$$

onde $m$ é o número de variáveis.

Desta equação (3.2) se obtém que germes simples podem ser encontrados se: $(m-2)\left(n^{2}+n\right)<m^{2}$. Uma superfície simples Cohen-Macaulay de codimensão dois em $\left(\mathbb{C}^{4}, 0\right)$, por exemplo, só pode ser dada por uma matriz $2 \times 3$.

Por argumentos similares podemos demonstrar os seguintes corolários. 
Corolário 3.6. Seja $M \in \mathcal{M a t} t_{3 \times 2}(\mathbb{C}\{x, y, z\})$ com entradas no ideal maximal, e sejam $f_{1}, f_{2}, f_{3}$ seus menores maximais. Se $j^{2}\left(f_{1}\right)=j^{2}\left(f_{2}\right)=0$, então o germe definido por $M$ não pode ser simples.

O seguinte corolário fornece uma lista de singularidades que não são simples.

Corolário 3.7. Seja $(D, a)$ um dos tipos de germes da Tabela (3.1), página (62). Então a matriz quase-homogênea de tipo $(D, a)$ não é simples.

Demonstração: Para os germes das linhas 3 - 9 e 12 - 13, a condição do Lema (3.3) é satisfeita. Assim o germe não pode ser simples. De fato: para o caso (3) temos que $a=(1,1,1)$ e se pode calcular facilmete que $D=\left(\begin{array}{ll}3 & 2\end{array}\right)$.

Calculamos então $\operatorname{dim} G_{0}$, temos que:

- $r(a, 3)=10$, pois os monômios de grau 3 com respeito aos pesos $a=(1,1,1)$ são: $x^{3}, y^{3}, z^{3}, x^{2} y, x^{2} z, x y^{2}, x z^{2}, y^{2} z, y z^{2}, x y z$.

- $r(a, 2)=6$, pois os únicos monômios de grau 2 com respeito aos pesos $a=(1,1,1)$ são: $x^{2}, y^{2}, z^{2}, x y, x z, z y$.

Então temos que $\operatorname{dim} G_{0}=r(a, 3)+r(a, 2)=16$. Agora calculamos: $s_{1}, s_{2}$ e $s_{3}$

- $s_{1}=9$, pois $i=1, \forall j$ e $1 \leq j \leq 3$

- $s_{2}=4$,

- $s_{3}=1$.

Assim $\sum_{i=1}^{3} s_{i}=14$

Logo $\operatorname{dim} G_{0} \geq \sum_{i=1}^{3} s_{i}-1$ e portanto $M$ não pode ser simples. Os outros casos são análogos.

Para as linhas 1,2, 10 e 11 existe outra relação entre os geradores do núcleo da função definida anteriormente, $G_{0} \rightarrow T^{1}(M)$, de fato:

Nas linhas 1 e 2 temos que: $z \frac{\partial M}{\partial z}=\left(\begin{array}{ll}0 & z\end{array}\right)=S_{22}$.

Nas linhas 10 e 11 temos que: $z \frac{\partial M}{\partial z}=\left(\begin{array}{ccc}z & 0 & 0 \\ 0 & 0 & 0\end{array}\right)=S_{11}$.

Assim para os casos mencionados acima temos que $\sum_{i j} r\left(a, D_{i j}\right) \geq s_{1}+s_{2}+s_{3}-2$, e pelo Lema (3.3) temos que o germe não é simples. 


\begin{tabular}{|c|c|c|c|c|c|}
\hline Forma normal da matriz genérica & $\tau$ & a & $\mathrm{D}$ & $\operatorname{Dim} G_{0}$ & $\sum_{i=1}^{3} s_{i}$ \\
\hline$\left(x^{3}+a x^{2} y^{2}+y^{6} z\right)$ & 10 & $(2,1,4)$ & $\left(\begin{array}{ll}6 & 4\end{array}\right)$ & 10 & 12 \\
\hline$\left(x^{4}+a x^{2} y^{2}+y^{4} z\right)$ & 9 & $(1,1,3)$ & $\left(\begin{array}{ll}4 & 3\end{array}\right)$ & 12 & 14 \\
\hline$\left(x^{3}+y^{3}+a x y z+b x z^{2} y^{2}+z^{2}+c x^{2}\right)$ & 13 & $(1,1,1)$ & $\left(\begin{array}{ll}3 & 2\end{array}\right)$ & 16 & 15 \\
\hline$\left(\begin{array}{cc}x^{2}+y z+z^{4} & \left.x y+a z^{5}\right)\end{array}\right.$ & 13 & $(2,3,1)$ & $\left(\begin{array}{ll}4 & 5\end{array}\right)$ & 9 & 10 \\
\hline$\left(\begin{array}{ll}x^{2}+z^{4} & y^{2}+a x z+z^{3}\end{array}\right)$ & 11 & $(4,3,2)$ & $\left(\begin{array}{ll}8 & 6\end{array}\right)$ & 7 & 8 \\
\hline$\left(x y+z^{3} y z+a x z^{2}+z^{3}\right)$ & 10 & $(1,2,1)$ & $\left(\begin{array}{ll}3 & 3\end{array}\right)$ & 12 & 13 \\
\hline$\left(\begin{array}{ccc}z+x^{3} & y & x \\
a x^{4} & z & y\end{array}\right)$ & 9 & $(1,3,2)$ & $\left(\begin{array}{lll}3 & 2 & 1 \\
4 & 3 & 2\end{array}\right)$ & 15 & 16 \\
\hline$\left(\begin{array}{ccc}z & x^{2}+a z^{2} & y \\
x & y & x^{2}-z^{2}\end{array}\right)$ & 8 & $(1,2,1)$ & $\left(\begin{array}{lll}1 & 2 & 2 \\
1 & 2 & 2\end{array}\right)$ & 20 & 21 \\
\hline$\left(\begin{array}{ccc}z & x & y \\
0 & y+a x^{2} & x^{3}-z^{2}\end{array}\right)$ & 11 & $(2,4,3)$ & $\left(\begin{array}{lll}3 & 2 & 4 \\
2 & 4 & 6\end{array}\right)$ & 10 & 11 \\
\hline$\left(\begin{array}{ccc}z & x^{3}+a x y^{2} & y^{3} \\
0 & y & x\end{array}\right)$ & 11 & $(2,2,5)$ & $\left(\begin{array}{lll}5 & 6 & 6 \\
6 & 2 & 2\end{array}\right)$ & 13 & 15 \\
\hline$\left(\begin{array}{ccc}z & x^{2}+a y^{4} & y^{5} \\
0 & y & x\end{array}\right)$ & 12 & $(4,2,7)$ & $\left(\begin{array}{ccc}7 & 8 & 10 \\
1 & 2 & 4\end{array}\right)$ & 10 & 12 \\
\hline$\left(\begin{array}{ccc}x & y & z \\
y z & x z+a z^{3} & y\end{array}\right)$ & 10 & $(2,2,1)$ & $\left(\begin{array}{lll}2 & 2 & 1 \\
3 & 3 & 2\end{array}\right)$ & 16 & 17 \\
\hline$\left(\begin{array}{ccc}x & y & z \\
z^{2} & x^{3}+a x^{2} z+b x z^{2}+c z^{3} & y\end{array}\right)$ & 13 & $(1,2,1)$ & $\left(\begin{array}{lll}1 & 2 & 1 \\
2 & 3 & 2\end{array}\right)$ & 22 & 21 \\
\hline
\end{tabular}

Tabela 3.1: Curvas espaciais não simples 
Corolário 3.8. Se $(D, a)$ é um dos tipos de matrizes quase-homogêneas da Tabela (3.1) e $N$ é outra matriz $3 \times 2$ com entradas no ideal maximal de $\mathbb{C}\{x, y, z\}$, satisfazendo $v_{(D, a)}(N) \geq 0$, então $N$ não pode ser simples.

Demonstração: Considere $N=j_{a} N+\widetilde{N}$ onde $j_{a} N$ é a parte de $N$ que tem peso relativo igual a zero, ou seja $v_{D, a}\left(j_{a} N\right)=0$. Seja $M$ uma matriz $3 \times 2$ quase-homogênea de tipo $(D, a)$ tal que $j_{a} N+t M$ é uma matriz genérica quase-homogênea de tipo $(D, a)$ para $t$ pequeno. Portanto, podemos assumir que o número de Tjurina de $j_{a} N+t M$ é finito. Então $v_{(D, a)}(\widetilde{N})>v_{(D, a)}(N) \geq 0$, portanto $\beta=0^{1}$. Como $\operatorname{dim} T^{1}(N)<\infty$ pelo Corolário (2.35) temos que $N+t M \sim_{\mathcal{G}(n)} j_{a} N+t M$. Assim $N$ não pode ser simples, pois $N$ deforma $j_{a} N+t M$, que não é simples.

\subsection{Candidatos para Curvas Espaciais Simples}

Usando as técnicas estudadas nas seções anteriores, apresentaremos nesta seção uma lista de singularidades que são candidatos a curvas espaciais simples que não são intersecções completas.

Proposição 3.9. Os germes de curvas definidas pelos menores maximais das matrizes nas Tabelas (3.2) e (3.3) são candidatos para singularidades simples.

Aqui apresentaremos uma visão global dos passos da prova.

Sabemos que equivalência de germes de matrizes implica equivalência dos 1- jatos das respectivas matrizes, assim primeiro classificaremos os 1- jatos.

Os passos que trabalharemos são:

(I) Classificação de 3 pontos em $\mathbb{P}^{2}$.

(II) Classificação de 1-jatos da matriz de representação.

(III) Procurar candidatos em cada um dos casos do item (II).

Definição 3.10. Se $f \in \mathbb{C}\{x\}$ é um polinômio homogêneo de grau d, então $f\left(\lambda x_{0}, \ldots, \lambda x_{m}\right)=$ $\lambda^{d} f\left(x_{0}, \ldots, x_{m}\right)$ para cada $\lambda \in \mathbb{C}$. Assim o conjunto:

$$
V(I)=\left\{p \in \mathbb{P}^{m} / f(p)=0\right\}
$$

\footnotetext{
${ }^{1}$ Definido na página 52
} 


\begin{tabular}{|c|c|c|c|c|c|}
\hline Tipo & $\begin{array}{l}\text { Apresentação } \\
\text { da Matriz }\end{array}$ & Condiçõos & $a$ & $\mu$ & $\tau$ \\
\hline$A_{k-3} \vee L$ & $\left(\begin{array}{ccc}z & y & x^{k-3} \\
0 & x & y\end{array}\right)$ & $\begin{array}{l}k \geq 4, \quad k \text { par } \\
k \geq 4, \quad k \text { impar }\end{array}$ & $\begin{array}{c}\left(1, \frac{k-2}{2}, \frac{k-2}{2}\right) \\
(1, k-2, k-2)\end{array}$ & $\begin{array}{l}k-2 \\
k-2\end{array}$ & $\begin{array}{l}k-1 \\
k-1\end{array}$ \\
\hline$E_{6}(1)$ & $\left(\begin{array}{ccc}z & y & x^{2} \\
x & z & y\end{array}\right)$ & & $(3,5,4)$ & 4 & 5 \\
\hline$E_{7}(1)$ & $\left(\begin{array}{ccc}z+x^{2} & y & x \\
0 & z & y\end{array}\right)$ & & $(2,3,4)$ & 5 & 6 \\
\hline$E_{8}(1)$ & $\left(\begin{array}{ccc}z & y & x^{3} \\
x & z & y\end{array}\right)$ & & $(3,7,5)$ & 6 & 7 \\
\hline$J_{2, k}(2)$ & $\left(\begin{array}{ccc}z+x^{2} & y & x^{k+2} \\
0 & z & y\end{array}\right)$ & $\begin{array}{l}k=0 \\
k=1\end{array}$ & $\begin{array}{l}(1,2,2) \\
(2,5,4)\end{array}$ & $\begin{array}{l}6 \\
7\end{array}$ & $\begin{array}{l}7 \\
8\end{array}$ \\
\hline$E_{12}(2)$ & $\left(\begin{array}{ccc}z & y & x^{3} \\
x^{2} & z & y\end{array}\right)$ & & $(3,8,7)$ & 8 & 9 \\
\hline
\end{tabular}

Tabela 3.2: Curvas espaciais de mutiplicidade 3 


\begin{tabular}{|c|c|c|c|c|c|}
\hline Tipo & $\begin{array}{l}\text { Apresentação } \\
\text { da Matriz }\end{array}$ & Condições & $a$ & $\mu$ & $\tau$ \\
\hline$D_{k+4} \vee L$ & $\left(\begin{array}{ccc}z & 0 & x^{k+2}-y^{2} \\
0 & x & y\end{array}\right)$ & $\begin{array}{l}k \geq 4, \quad k \text { par } \\
k \geq 4, \quad k \text { impar }\end{array}$ & $\begin{array}{c}\left(1, \frac{k+2}{2}, \frac{k+2}{2}\right) \\
(2, k+2, k+2)\end{array}$ & $\begin{array}{l}k+5 \\
k+5\end{array}$ & $\begin{array}{l}k+6 \\
k+6\end{array}$ \\
\hline$E_{6} \vee L$ & $\left(\begin{array}{ccc}z & -y^{2} & -x^{3} \\
0 & x & y\end{array}\right)$ & & $(3,4,5)$ & 7 & 8 \\
\hline$E_{7} \vee L$ & $\left(\begin{array}{ccc}z & x^{3}-y^{2} & 0 \\
0 & x & y\end{array}\right)$ & & $(2,3,4)$ & 8 & 9 \\
\hline$E_{8} \vee L$ & $\left(\begin{array}{ccc}z & -y^{2} & -x^{4} \\
0 & x & y\end{array}\right)$ & & $(3,5,7)$ & 9 & 10 \\
\hline$S_{6}^{*}$ & $\left(\begin{array}{ccc}z & x & y \\
0 & y & x^{2}-z^{2}\end{array}\right.$ & & $(2,3,2)$ & 6 & 7 \\
\hline$T_{7}^{*}$ & $\left(\begin{array}{ccc}z & x & y \\
0 & y & x^{2}-z^{3}\end{array}\right)$ & & $(6,9,4)$ & 7 & 8 \\
\hline$U_{7}^{*}$ & $\left(\begin{array}{ccc}z & x y & x^{2} \\
x & z & y\end{array}\right)$ & & $(3,4,5)$ & 7 & 8 \\
\hline$W_{8}^{*}$ & $\left(\begin{array}{ccc}z & y^{2} & x^{2} \\
x & z & y\end{array}\right)$ & & $(4,5,7)$ & 8 & 9 \\
\hline
\end{tabular}

Tabela 3.3: Curvas espaciais de mutiplicidade 4 
está bem definido. Mas geralmente, se $I \subset \mathbb{C}\{x\}$ é um ideal homogêneo (i.e I é gerado por polinômios homogêneos) então o conjunto

$$
V(I)=\left\{p \in \mathbb{P}^{m} / f(p)=0 \text { para todo } f \in I\right\}
$$

está bem definido e é chamado conjunto algébrico projetivo definido por I.

\subsection{Passo I: Classificação de Três Pontos em $\mathbb{P}^{2}$.}

Seja $V(I) \subseteq \mathbb{P}^{2}$ um conjunto algébrico 0- dimensional de multiplicidade 3 . Isto é $V(I)$ consiste de três pontos (contando suas multiplicidades).

1. Se $V(I)$ pode ser mergulhado em $\mathbb{P}^{1}$, então $I$ é da forma $I=(f(x, y), z)$; onde $f(x, y)$ é o gerador do ideal principal descrevendo estes três pontos em $\mathbb{P}^{1}$. Além disso, I é equivalente (por [18], capítulo 2) a um dos seguintes casos:

- 3 pontos distintos

$I=\left(x^{3}-x y^{2}, z\right)$

- 1 ponto de multiplicidade 2 e mais um ponto simples

$I=\left(x^{2} y, z\right)$

- 1 ponto de multiplicidade 3

$I=\left(x^{3}, z\right)$

2. Se $V(I)$ não pode ser mergulhado em $\mathbb{P}^{1}$, então temos que estudar três situações:

- 3 Pontos distintos:

Em $\mathbb{P}^{2}$ as coordenadas podem ser escolhidas tais que estes pontos sejam (1 : $0: 0),(0: 1: 0)$ e $(0: 0: 1)$. Logo $V\left(I_{1}\right)=\{(1: 0: 0)\}, V\left(I_{2}\right)=\{(0:$ $1: 0)\}, V\left(I_{3}\right)=\{(0: 0: 1)\}$ então $I_{1}=(y, z), I_{2}=(x, z), I_{3}=(x, y)$. Logo $V\left(I_{1} \cdot I_{2} \cdot I_{3}\right)=V\left(I_{1}\right) \cup V\left(I_{2}\right) \cup V\left(I_{3}\right)$, por propriedades de conjuntos algébricos. Seja $I=I_{1} \cdot I_{2} \cdot I_{3}$, então $V(I)=\{(1: 0: 0),(0: 1: 0),(0: 0: 1)\}$. Assim $I$ é da forma $I=(x z, y z, x y)$.

- 1 ponto de multiplicidade 2 e mais um ponto simples.

Neste caso podemos escolher coordenadas tais que o ponto de multiplicidade 2 seja ( $1: 0: 0)$, e o ponto de multiplicidade 1 seja $(0: 0: 1)$. A direção do espaço tangente do ponto não pode ser $y=0$, pois estamos considerando que os três pontos não são mergulhados em $\mathbb{P}^{1}$. Além disso podemos escolher esta direção como $z=0$. Considerando $V\left(I_{1}\right)=\{(1: 0: 0)\}, V\left(I_{2}\right)=$ 
$\{(0: 0: 1)\}$ então $I_{1}=(y, z), I_{2}=(x, y), \operatorname{logo} I=I_{1} \cdot I_{2}=\left(y x, y^{2}, z x, z y\right) \mathrm{e}$ assim $I$ é da forma $I=\left(x z, y z, y^{2}\right)$.

- 1 ponto de multiplicidade 3 .

Por uma escolha apropriada de coordenadas, podemos supor que o ponto é $(1: 0: 0)$. Agora consideramos a aplicação $h:(1: y: z) \longmapsto(y, z)$ e obtemos os seguintes dois casos para o anel local:

$$
\begin{aligned}
\frac{\mathbb{C}\{y, z\}}{h(I)} \cong \frac{\mathbb{C}\{y, z\}}{\left(y z, y^{2}, z^{2}\right)} \Rightarrow I=\left(y z, y^{2}, z^{2}\right) \\
\frac{\mathbb{C}\{y, z\}}{h(I)} \cong \frac{\mathbb{C}\{y, z\}}{\left(y, z^{3}\right)} \cong \frac{\mathbb{C}\{y, z\}}{\left(y z, y^{2}, z^{2}-y\right)} \Rightarrow I=\left(y z, y^{2}, z^{2}-x y\right)
\end{aligned}
$$

Temos que o ideal $\left\langle y z, y^{2}, z^{2}-y\right\rangle=\left\langle z^{3}, z^{2}-y\right\rangle$, o isomorfismo em (3.4) é dado por:

$$
\begin{aligned}
\Phi: \frac{\mathbb{C}\{y, z\}}{\left(y, z^{3}\right)} & \longrightarrow \frac{\mathbb{C}\{y, z\}}{\left(z^{3}, z^{2}-y\right)} \\
z & \longmapsto z \\
y & \longmapsto z^{2}-y,
\end{aligned}
$$

cujo inverso é dado pelo seguinte homomorfismo:

$$
\begin{aligned}
\Psi: \frac{\mathbb{C}\{y, z\}}{\left(z^{3}, z^{2}-y\right)} & \longrightarrow \frac{\mathbb{C}\{y, z\}}{\left(y, z^{3}\right)} \\
z & \longmapsto z \\
y & \longmapsto z^{2}-y .
\end{aligned}
$$

\subsection{Passo II: Classificação dos 1-Jatos da Matriz de Repre- sentação.}

O seguinte lema é um lema técnico muito usual que pode ser provado usando transformações de linhas e colunas.

Lema 3.11. Se $M \in \mathcal{M a t}_{3 \times 2}(\mathbb{C}\{y, z\})$ é da forma

$$
\left(\begin{array}{lcr}
z & y & 0 \\
a_{1} y+b_{1} z y & a_{2} y+b_{2} z & a_{3} y+b_{3} z
\end{array}\right) a_{i}, b_{i} \in \mathbb{C}
$$

e para toda matriz na órbita de $M$ temos que nenhum menor maximal é nulo (quer dizer que $b_{3}=0, a_{3} \neq 0$ ou $\left.b_{3} \neq 0, a_{1} b_{3}^{2}-a_{3} b_{1} b_{3}+a_{2} a_{3} b_{3}-b_{2} a_{3}^{2} \neq 0\right)$, então $M$ é $\mathcal{G}(n)$ equivalente à 
matriz.

$$
\left(\begin{array}{lll}
z & y & 0 \\
y & 0 & z
\end{array}\right)
$$

\section{Demonstração:}

- Suponha que $b_{3}=0, a_{3} \neq 0$, daremos os passos para as transformações de linhas e colunas:
1. $\left(\begin{array}{ccc}z & y & 0 \\ a_{1} y & \left(a_{2}-b_{1}\right) y+b_{2} z & a_{3} y\end{array}\right)$
2. $\left(\begin{array}{ccc}z & a_{3} y & 0 \\ a_{1} y & a_{3} b_{2} z & a_{3} y\end{array}\right)$
3. $\left(\begin{array}{ccc}a_{3} z & a_{3} y & 0 \\ 0 & a_{3} b_{2} z & a_{3} y\end{array}\right)$
4. $\left(\begin{array}{ccc}y & z & 0 \\ b_{2} z & 0 & y\end{array}\right)$
5. $\left(\begin{array}{ccc}b_{2} y & b_{2} z & 0 \\ b_{2} z & 0 & b_{2} y\end{array}\right)$

Seja a seguinte mudança de coordenadas:

$$
\begin{aligned}
\phi: \mathbb{C}\{y, z\} & \longrightarrow \mathbb{C}\{y, z\} \\
z & \longmapsto b_{2} y \\
y & \longmapsto b_{2} z .
\end{aligned}
$$

$\operatorname{Logo} \phi^{*}\left(\left(\begin{array}{ccc}z & y & 0 \\ y & 0 & z\end{array}\right)\right)=\left(\begin{array}{ccc}b_{2} y & b_{2} z & 0 \\ b_{2} z & 0 & b_{2} y\end{array}\right)$ e assim obtemos o resultado.

- Suponha que $b_{3} \neq 0, a_{1} b_{3}^{2}-a_{3} b_{1} b_{3}+a_{2} a_{3} b_{3}-b_{2} a_{3}^{2} \neq 0$. Daremos os passos para as transformações de linhas e colunas:
1. $\left(\begin{array}{lrr}z & y & 0 \\ a_{1} y & \left(a_{2}-b_{1}\right) y+b_{2} z & a_{3} y+b_{3} z\end{array}\right)$
2. $\left(\begin{array}{ccr}b_{3} z & a_{3} y & 0 \\ a_{1} b_{3} y & a_{3}\left(a_{2}-b_{1}\right) y+b_{2} a_{3} z & a_{3} y+b_{3} z\end{array}\right)$
3. $\left(\begin{array}{lcr}b_{3} z+a_{3} y & a_{3} y & 0 \\ \left(a_{1} b_{3}+a_{3} a_{2}-a_{3} b_{1}\right) y+a_{3} b_{2} z & a_{3}\left(a_{2}-b_{1}\right) y+a_{3} b_{2} z & a_{3} y+b_{3} z\end{array}\right)$ 

4. $\left(\begin{array}{ccr}b_{3} z+a_{3} y & a_{3} b_{3} y & 0 \\ \left(a_{1} b_{3}+a_{3} a_{2}-a_{3} b_{1}\right) y+a_{3} b_{2} z & \left(b_{3} a_{3} a_{2}-b_{3} a_{3} b_{1}-a_{3}^{2} b_{2}\right) y & a_{3} y+b_{3} z\end{array}\right)$
5. $\left(\begin{array}{lcr}b_{3} z+a_{3} y & a_{3} b_{3} y & 0 \\ \left(a_{3}^{3} b_{2}+a_{1} a_{3} b_{3}^{2}\right) y+\left(-b_{3}^{2} a_{3} a_{2}+b_{3}^{2} a_{3} b_{1}+a_{3}^{2} b_{2} b_{3}+b_{3} a_{3}^{2} b_{2}\right) z & 0 & a_{3} y+b_{3} z\end{array}\right)$
6. $\left(\begin{array}{lcr}b_{3}\left(b_{3} z+a_{3} y\right) & a_{3} y & 0 \\ b_{3}\left(b_{3} a_{3} a_{2}-b_{3} a_{3} b_{1}-a_{3}^{2} b_{3}+a_{1} b_{3}^{2}\right) y & 0 & a_{3} y+b_{3} z\end{array}\right)$
7. $\left(\begin{array}{lcr}b_{3} z+a_{3} y & a_{3}\left(b_{3} a_{3} a_{2}-b_{3} a_{3} b_{1}-a_{3}^{2} b_{3}+a_{1} b_{3}^{2}\right) y & 0 \\ a_{3}\left(b_{3} a_{3} a_{2}-b_{3} a_{3} b_{1}-a_{3}^{2} b_{3}+a_{1} b_{3}^{2}\right) y & 0 & b_{3} z+a_{3} y\end{array}\right)$

Seja a seguinte mudança de coordenadas:

$$
\begin{aligned}
\phi: \mathbb{C}\{y, z\} & \longrightarrow \mathbb{C}\{y, z\} \\
z & \longmapsto b_{3} z+a_{3} y \\
y & \longmapsto a_{3}\left(b_{3} a_{3} a_{2}-b_{3} a_{3} b_{1}-a_{3}^{2} b_{3}+a_{1} b_{3}^{2}\right) y
\end{aligned}
$$

Logo: $\phi^{*}\left(\left(\begin{array}{ccc}z & y & 0 \\ y & 0 & z\end{array}\right)\right)=$

$$
\left(\begin{array}{lcr}
b_{3} z+a_{3} y & a_{3}\left(b_{3} a_{3} a_{2}-b_{3} a_{3} b_{1}-a_{3}^{2} b_{3}+a_{1} b_{3}^{2}\right) y & 0 \\
a_{3}\left(b_{3} a_{3} a_{2}-b_{3} a_{3} b_{1}-a_{3}^{2} b_{3}+a_{1} b_{3}^{2}\right) y & 0 & b_{3} z+a_{3} y
\end{array}\right) .
$$

Daí obtemos o resultado.

Lema 3.12. Seja $M \in \mathcal{M a t}_{3 \times 2}(\mathbb{C}\{x, y, z\})$ definindo um germe de curva que não é equivalente a uma intersecção completa. Então o 2-jato de seus menores maximais $f=\left(f_{1}, f_{2}, f_{3}\right)$ é equivalente a uma das funções seguintes:
1. $j^{2} f \sim_{\mathcal{K}}(z x, z y, x y)$
5. $j^{2} f \sim_{\mathcal{K}}(z x, z y, 0)$
2. $j^{2} f \sim_{\mathcal{K}}\left(z x, z y, y^{2}\right)$
6. $j^{2} f \sim_{\mathcal{K}}\left(z y, y^{2}, 0\right)$
3. $j^{2} f \sim_{\mathcal{K}}\left(z y, y^{2}, z^{2}-x y\right)$
7. $j^{2} f \sim \sim_{\mathcal{K}}\left(z x, z y, z^{2}\right)$
4. $j^{2} f \sim_{\mathcal{K}}\left(z y, y^{2}, z^{2}\right)$
8. $j^{2} f \sim_{\mathcal{K}}(q, 0,0)$,

onde q é uma forma quadrática arbitrária ou zero. Nos casos 7 e 8, M não pode definir uma singularidade simples.

Demonstração: Como $f$ não é $\mathcal{K}$-equivalente a uma intersecção completa, todas as entradas de $M$ estão no ideal maximal $\mathbf{m}$ de $\mathbb{C}\{x, y, z\}$. Temos que $j^{2} f$ é obtido pelos 
menores maximais da matriz $j^{1} M$. Vamos fazer esta classificação por casos:

1. Caso 1: $j^{2} f \neq 0, \forall 1 \leq i \leq 3$

Parte a: Estrutura da matriz Representação.

Suponha que $j^{1} M_{11} \neq 0$, então:

$$
j^{1} M=\left(\begin{array}{ccc}
j^{1} M_{11}(x, y, z) & j^{1} M_{12}(x, y, z) & j^{1} M_{13}(x, y, z) \\
j^{1} M_{21}(x, y, z) & j^{1} M_{22}(x, y, z) & j^{1} M_{23}(x, y, z)
\end{array}\right) .
$$

Considere a seguinte mudança de coordenadas $\left(\phi \in \mathcal{R}_{m}\right)$ :

$$
\begin{aligned}
\phi: \mathbb{C}\{x, y, z\} & \longrightarrow \mathbb{C}\{x, y, z\} \\
x & \longmapsto x \\
y & \longmapsto y \\
z & \longmapsto j^{1} M_{11}(x, y, z),
\end{aligned}
$$

então teríamos que:

$$
j^{1} M \sim_{\mathcal{G}(n)}\left(\begin{array}{ccc}
z & j^{1} \tilde{M}_{12}(x, y, z) & j^{1} \tilde{M}_{13}(x, y, z) \\
j^{1} \tilde{M}_{21}(x, y, z) & j^{1} \tilde{M}_{22}(x, y, z) & j^{1} \tilde{M}_{23}(x, y, z)
\end{array}\right) .
$$

Fazendo transformações de linhas e colunas podemos eliminar a variável $z$ em $j^{1} \tilde{M}_{12}(x, y, z), j^{1} \tilde{M}_{13}(x, y, z)$ e $j^{1} \tilde{M}_{13}(x, y, z)$. Sem perda de generalidade podemos considerar as mesmas notações para as entradas de $j^{1} M$ para não carregar a notação, obtendo:

$$
j^{1} M \sim_{\mathcal{G}(n)}\left(\begin{array}{ccc}
z & j^{1} \tilde{M}_{12}(x, y) & j^{1} \tilde{M}_{13}(x, y) \\
j^{1} \tilde{M}_{21}(x, y) & j^{1} \tilde{M}_{22}(x, y, z) & j^{1} \tilde{M}_{23}(x, y, z)
\end{array}\right) .
$$

Além disso, temos que nenhum dos menores maximais se anulam portanto $j^{1} \tilde{M}_{12}(x, y) \neq 0$ ou $j^{1} \tilde{M}_{13}(x, y) \neq 0$. Suponha que $j^{1} \tilde{M}_{12}(x, y), j^{1} \tilde{M}_{13}(x, y)$ são linearmente dependentes. Então fazendo transformações de colunas temos que:

$$
j^{1} M \sim_{\mathcal{G}(n)}\left(\begin{array}{ccc}
z & j^{1} \tilde{M}_{12}(x, y) & 0 \\
j^{1} \tilde{M}_{21}(x, y) & j^{1} \tilde{M}_{22}(x, y, z) & j^{1} \tilde{M}_{23}(x, y, z)
\end{array}\right) .
$$


Logo, consideremos a seguinte mudança de coordenadas:

$$
\begin{aligned}
\phi: \mathbb{C}\{x, y, z\} & \longrightarrow \mathbb{C}\{x, y, z\} \\
z & \longmapsto z \\
x & \longmapsto x \\
y & \longmapsto j^{1} \tilde{M}_{12}(x, y) .
\end{aligned}
$$

Assim:

$$
j^{1} M \sim_{\mathcal{G}(n)}\left(\begin{array}{ccc}
z & y & 0 \\
j^{1} \tilde{M}_{21}(x, y) & j^{1} \tilde{M}_{22}(x, y, z) & j^{1} \tilde{M}_{23}(x, y, z)
\end{array}\right) .
$$

Agora suponha que $j^{1} \tilde{M}_{12}(x, y), j^{1} \tilde{M}_{13}(x, y)$ são linearmente independentes. Fazendo a seguinte mudança de coordenadas:

$$
\begin{aligned}
\phi: \mathbb{C}\{x, y, z\} & \longrightarrow \mathbb{C}\{x, y, z\} \\
z & \longmapsto z \\
x & \longmapsto j^{1} \tilde{M}_{13}(x, y) \\
y & \longmapsto j^{1} \tilde{M}_{12}(x, y),
\end{aligned}
$$

temos que:

$$
j^{1} M \sim_{\mathcal{G}(n)}\left(\begin{array}{ccc}
z & y & x \\
j^{1} \tilde{M}_{21}(x, y) & j^{1} \tilde{M}_{22}(x, y, z) & j^{1} \tilde{M}_{23}(x, y, z)
\end{array}\right) .
$$

Destes casos concluímos que:

$$
j^{1} M \sim_{\mathcal{G}(n)}\left(\begin{array}{ccc}
z & y & \alpha x \\
j^{1} \tilde{M}_{21}(x, y) & j^{1} \tilde{M}_{22}(x, y, z) & j^{1} \tilde{M}_{23}(x, y, z)
\end{array}\right) .
$$

onde $\alpha \in\{0,1\}$.

Para $\alpha=0, j^{1} M \sim_{\mathcal{G}(n)}\left(\begin{array}{ccc}z & y & 0 \\ a_{1} x+b_{1} y & a_{2} x+b_{2} y+c_{2} z & a_{3} x+b_{3} y+c_{3} z\end{array}\right)$. Vamos considerar as seguintes mudanças de coordenadas.

(a) Suponha que $a_{3} \neq 0$.

$$
\begin{aligned}
\phi_{1}: \mathbb{C}\{x, y, z\} & \longrightarrow \mathbb{C}\{x, y, z\} \\
x & \longmapsto a_{3} x+b_{3} y+c_{3} z
\end{aligned}
$$


(b) suponha que $a_{3}=0$ e $a_{2} \neq 0$ então:

$$
\begin{aligned}
\phi_{2}:\{x, y, z\} & \longrightarrow\{x, y, z\} \\
x & \longmapsto a_{2} x+b_{2} y+c_{2} z
\end{aligned}
$$

(c) suponha que $a_{1} \neq 0, a_{2}=0, a_{3}=0$

$$
\begin{aligned}
\phi_{3}:\{x, y, z\} & \longrightarrow\{x, y, z\} \\
x & \longmapsto a_{1} x+b_{1} y
\end{aligned}
$$

(d) suponha que $a_{1}=0, a_{2}=0, a_{3}=0$

Para qualquer destes casos temos que $j^{1} M$ é equivalente a:
(a) $\left(\begin{array}{lll}z & y & 0 \\ * & * & x\end{array}\right)$
(c) $\left(\begin{array}{lll}z & y & 0 \\ x & \star & \star\end{array}\right)$
(b) $\left(\begin{array}{lll}z & y & 0 \\ * & x & \star\end{array}\right)$
(d) $\left(\begin{array}{ccc}z & y & 0 \\ \star & \star & \star\end{array}\right)$,

onde $*$ denota uma forma linear em $x, y$ e $z, \star$ denota uma forma linear em $y \mathrm{e} z$.

No caso $(d)$ pelo Lema (3.11) temos que:

$$
j^{1} M \sim_{\mathcal{G}(n)}\left(\begin{array}{ccc}
z & y & 0 \\
y & 0 & z
\end{array}\right)
$$

e portanto $\underline{j^{2} f \sim_{\mathcal{K}}\left(z y, z^{2}, y^{2}\right)}$.

No caso $(c)$ temos que:

$$
j^{1} M \sim_{\mathcal{G}(n)}\left(\begin{array}{ccc}
z & y & 0 \\
x & a_{1} y+b_{1} z & a_{2} y+b_{2} z
\end{array}\right) .
$$

Suponha que $b_{1} \neq 0$ e $b_{2} \neq 0$, fazendo transformação de linhas e colunas a esta última matriz temos:

$$
j^{1} M \sim_{\mathcal{G}(n)}\left(\begin{array}{ccc}
z & b_{2} y & 0 \\
x & \left(a_{1} b_{2}-a_{2} b_{1}\right) z & a_{2} y+b_{2} z
\end{array}\right) .
$$

Suponha agora que $a_{1} b_{2}-a_{2} b_{1} \neq 0$, então: 


$$
j^{1} M \sim_{\mathcal{G}(n)}\left(\begin{array}{ccc}
z & b_{2} y & 0 \\
b_{2} x-\left(a_{1} b_{2}-a_{2} b_{1}\right) z & 0 & a_{2} y+b_{2} z
\end{array}\right) .
$$

Considere agora a seguinte mudança de coordenadas:

$$
\begin{aligned}
& \phi: \mathbb{C}\{x, y, z\} \quad \rightarrow \quad \mathbb{C}\{x, y, z\} \\
& x \quad \longmapsto b_{2} x-\left(a_{1} b_{2}-a_{2} b_{1}\right) z \\
& j^{1} M \sim_{\mathcal{G}(n)}\left(\begin{array}{ccc}
z & b_{2} y & 0 \\
x & 0 & a_{2} y+b_{2} z
\end{array}\right) \sim_{\mathcal{G}(n)}\left(\begin{array}{ccc}
z & y & 0 \\
x & 0 & a_{2} y+z
\end{array}\right) .
\end{aligned}
$$

Vamos usar o Lema de Mather (1.15) para mostrar que:

$$
\left(\begin{array}{ccc}
z & y & 0 \\
x & 0 & a_{2} y+z
\end{array}\right) \sim_{\mathcal{G}(n)}\left(\begin{array}{ccc}
z & y & 0 \\
x & 0 & z
\end{array}\right) .
$$

Seja $\mathcal{M}=\left\{\left(\begin{array}{ccc}z & y & 0 \\ x & 0 & a_{2} y+z\end{array}\right) / \forall a_{2} \in \mathbb{C}\right\}$, uma subvariedade de $J^{1} \mathcal{M} a t_{(n+1) n}(\mathbb{C}\{x, y, z\})$. $\mathcal{M}$ é uma variedade conexa, cujo espaço tangente é e que:

$$
T_{M}(\mathcal{M})=\mathcal{O}_{3}\left\langle\left(\begin{array}{lll}
0 & 0 & 0 \\
0 & 0 & y
\end{array}\right)\right\rangle, \forall M \in \mathcal{M} .
$$

Por outro lado, o espaço tangente da órbita de $M \in \mathcal{M}$ é dado por:

$$
\begin{gathered}
T_{M} \mathcal{G}(n)(M)=\mathbf{m}_{3}\left\langle\left(\begin{array}{lll}
0 & 0 & 0 \\
1 & 0 & 0
\end{array}\right),\left(\begin{array}{lll}
0 & 1 & 0 \\
0 & 0 & a_{2}
\end{array}\right),\left(\begin{array}{lll}
1 & 0 & 0 \\
0 & 0 & 1
\end{array}\right)\right\rangle+\mathcal{O}_{3}\left\langle\left(\begin{array}{ccc}
z & y & 0 \\
0 & 0 & 0
\end{array}\right),\right. \\
\left(\begin{array}{lll}
0 & 0 & 0 \\
z & y & 0
\end{array}\right),\left(\begin{array}{ccc}
x & 0 & a_{2} y+z \\
0 & 0 & 0
\end{array}\right),\left(\begin{array}{ccc}
0 & 0 & 0 \\
x & 0 & a_{2} y+z
\end{array}\right),\left(\begin{array}{lll}
z & 0 & 0 \\
x & 0 & 0
\end{array}\right),\left(\begin{array}{lll}
0 & z & 0 \\
0 & x & 0
\end{array}\right), \\
\left(\begin{array}{lll}
0 & 0 & z \\
0 & 0 & x
\end{array}\right),\left(\begin{array}{ccc}
y & 0 & 0 \\
0 & 0 & 0
\end{array}\right),\left(\begin{array}{lll}
0 & y & 0 \\
0 & 0 & 0
\end{array}\right),\left(\begin{array}{ccc}
0 & 0 & y \\
0 & 0 & 0
\end{array}\right),\left(\begin{array}{ccc}
0 & 0 & 0 \\
a_{2} y+z & 0 & 0
\end{array}\right) \\
\left.,\left(\begin{array}{ccc}
0 & 0 & 0 \\
0 & a_{2} y+z & 0
\end{array}\right),\left(\begin{array}{ccc}
0 & 0 & 0 \\
0 & 0 & a_{2} y+z
\end{array}\right)\right\rangle .
\end{gathered}
$$

Logo:

$\left(\begin{array}{lll}0 & 0 & 0 \\ 0 & 0 & y\end{array}\right)=\left(\begin{array}{lll}y & 0 & 0 \\ 0 & 0 & y\end{array}\right)-\left(\begin{array}{lll}y & 0 & 0 \\ 0 & 0 & 0\end{array}\right) \in T_{M} \mathcal{G}_{n} M$, para qualquer $a_{2} \in \mathbb{C}$. 
Além disso, $\operatorname{dim} T_{M} \mathcal{G}_{n} M=17$ é constante , $\forall M \in \mathcal{M}$. Logo

$$
\begin{gathered}
\left(\begin{array}{ccc}
z & y & 0 \\
x & 0 & a_{2} y+z
\end{array}\right) \sim_{\mathcal{G}(n)}\left(\begin{array}{lll}
z & y & 0 \\
x & 0 & z
\end{array}\right) \\
\text { e assim } j^{1} M \sim_{\mathcal{G}(n)}\left(\begin{array}{ccc}
z & y & 0 \\
x & 0 & z
\end{array}\right) \sim_{\mathcal{G}(n)}\left(\begin{array}{ccc}
y & z & 0 \\
x & 0 & y
\end{array}\right) . \text { Portanto } \\
\underline{j^{2} f \sim_{\mathcal{K}}\left(z x, z y, y^{2}\right) .}
\end{gathered}
$$

Se $a_{1} b_{2}-a_{2} b_{1}=0$, então:

$$
j^{1} M \sim_{\mathcal{G}(n)}\left(\begin{array}{ccc}
z & b_{2} y & 0 \\
x & 0 & a_{2} y+b_{2} z
\end{array}\right)
$$

pelo caso anterior:

$$
\underline{j^{2} f \sim_{\mathcal{K}}\left(z x, z y, y^{2}\right)} .
$$

Suponha agora que $b_{1}=0$ ou $b_{2}=0$, para estes se obtém que:

Se $b_{1}=0$ e $b_{2}=0$ então $\underline{j^{2} f \sim_{\mathcal{K}}\left(z x, z y, z^{2}\right)}$.

Se $b_{1}=0, b_{2} \neq 0$, então $j^{1} M \sim_{\mathcal{G}(n)}\left(\begin{array}{ccc}z & y & 0 \\ x & 0 & a_{2} y+b_{2} z\end{array}\right) \sim_{\mathcal{G}(n)}^{b_{2} \neq 0}\left(\begin{array}{ccc}z & y & 0 \\ x & 0 & z\end{array}\right)$, assim $\underline{j^{2} f \sim_{\mathcal{K}}\left(z x, z y, y^{2}\right)}$

Se $b_{1} \neq 0, b_{2}=0$ então: $j^{1} M \sim_{\mathcal{G}(n)}\left(\begin{array}{ccc}z & y & 0 \\ x & a_{1} y+b_{z} & y\end{array}\right)$, $\operatorname{assim} \underline{j^{2} f \sim_{\mathcal{K}}\left(z y, y^{2}, z^{2}-x y\right)}$ ou $\underline{j^{2} f \sim_{\mathcal{K}}\left(z x, z y, z^{2}\right)}$.

No caso $(b)$ temos que $j^{1} M \sim_{\mathcal{G}(n)}\left(\begin{array}{ccc}z & y & 0 \\ a_{1} x+b_{1} y+c_{1} z & x & b_{2} y+c_{2} z\end{array}\right)$.

Suponha que $b_{1} \neq 0$ e $b_{2} \neq 0$.

Fazendo transformações de linhas e colunas vamos ter:

$$
j^{1} M \sim_{\mathcal{G}(n)}\left(\begin{array}{ccc}
b_{2} z & y & 0 \\
b_{2} a_{1} x+\left(c_{1} b_{2}-b_{1} c_{2}\right) z & x & y+c_{2} z
\end{array}\right) .
$$


Suponha que $c_{1} b_{2}-b_{1} c_{2} \neq 0$ então:

$$
j^{1} M \sim_{\mathcal{G}(n)}\left(\begin{array}{ccc}
b_{2} z-b_{2} a_{1} y & y & 0 \\
\left(c_{1} b_{2}-b_{1} c_{2}\right) z & x & y+c_{2} z
\end{array}\right)
$$

Considere a seguinte mudança de coordenadas:

$$
\begin{aligned}
\phi: \mathbb{C}\{x, y, z\} & \longrightarrow \mathbb{C}\{x, y, z\} \\
z & \longmapsto b_{2} z-b_{2} a_{1} y
\end{aligned}
$$

assim obtemos que:

$$
j^{1} M \sim_{\mathcal{G}(n)}\left(\begin{array}{ccc}
z & y & 0 \\
\tilde{b} y+\tilde{c} z & x & y+c_{2} z
\end{array}\right) .
$$

Logo, suponha que $\tilde{b} \neq 0$ então $j^{1} M \sim_{\mathcal{G}(n)}\left(\begin{array}{ccc}z & y & 0 \\ \left(\tilde{c}-\tilde{b} c_{2}\right) z & x & y+c_{2} z\end{array}\right)$. Suponha que $\left(\tilde{c}-\tilde{b} c_{2}\right) \neq 0$,

$$
j^{1} M \sim_{\mathcal{G}(n)}\left(\begin{array}{ccc}
z & y & 0 \\
0 & y-\left(\tilde{c}-\tilde{b} c_{2}\right) x & y+c_{2} z
\end{array}\right) .
$$

Considere agora a seguinte mudança de coordenadas:

$$
\begin{aligned}
\phi: \mathbb{C}\{x, y, z\} & \longrightarrow \mathbb{C}\{x, y, z\} \\
x & \longmapsto y-\left(\tilde{c}-\tilde{b} c_{2}\right) x
\end{aligned}
$$

Logo:

$$
j^{1} M \sim_{\mathcal{G}(n)}\left(\begin{array}{ccc}
z & y & 0 \\
0 & x & y+c_{2} z
\end{array}\right)
$$

Considere agora, $\mathcal{M}=\left\{\left(\begin{array}{ccc}z & y & 0 \\ 0 & x & y+c_{2} z\end{array}\right) / \tilde{c} \in \mathbb{C}\right\}$, que é $\mathcal{M}$ subvariedade conexa de $J^{1} \mathcal{M a t}_{(n+1) n}(\mathbb{C}\{x, y, z\})$. Vamos usar o Lema de Mather (1.15) para mostrar que $\mathcal{M}$ está contida em uma única órbita. De fato, calculamos o espaço tangente de $M \in \mathcal{M}$.

$$
T_{M} \mathcal{G}(n)(M)=\mathbf{m}_{3}\left\langle\left(\begin{array}{ccc}
0 & 0 & 0 \\
0 & 1 & 0
\end{array}\right),\left(\begin{array}{ccc}
0 & 1 & 0 \\
0 & 0 & 1
\end{array}\right),\left(\begin{array}{ccc}
1 & 0 & 0 \\
0 & 0 & \tilde{c}
\end{array}\right)\right\rangle+\mathcal{O}\left\langle\left(\begin{array}{ccc}
z & y & 0 \\
0 & 0 & 0
\end{array}\right),\right.
$$




$$
\begin{gathered}
\left(\begin{array}{ccc}
0 & 0 & 0 \\
z & y & 0
\end{array}\right),\left(\begin{array}{ccc}
0 & x & y+c_{2} z \\
0 & 0 & 0
\end{array}\right),\left(\begin{array}{ccc}
0 & 0 & 0 \\
0 & x & y+c_{2} z
\end{array}\right),\left(\begin{array}{ccc}
z & 0 & 0 \\
0 & 0 & 0
\end{array}\right),\left(\begin{array}{ccc}
0 & z & 0 \\
0 & 0 & 0
\end{array}\right), \\
\left(\begin{array}{ccc}
0 & 0 & z \\
0 & 0 & 0
\end{array}\right),\left(\begin{array}{ccc}
y & 0 & 0 \\
x & 0 & 0
\end{array}\right),\left(\begin{array}{ccc}
0 & y & 0 \\
0 & x & 0
\end{array}\right),\left(\begin{array}{ccc}
0 & 0 & y \\
0 & 0 & x
\end{array}\right),\left(\begin{array}{ccc}
0 & 0 & 0 \\
y+c_{2} z & 0 & 0
\end{array}\right), \\
\left.\left(\begin{array}{ccc}
0 & 0 & 0 \\
0 & y+c_{2} z & 0
\end{array}\right),\left(\begin{array}{ccc}
0 & 0 & 0 \\
0 & 0 & y+\tilde{c} z
\end{array}\right)\right\rangle,
\end{gathered}
$$

Temos que $T_{M}(\mathcal{M})=\mathcal{O}_{3}\left\langle\left(\begin{array}{lll}0 & 0 & 0 \\ 0 & 0 & z\end{array}\right)\right\rangle$, então:

$$
\left(\begin{array}{lll}
0 & 0 & 0 \\
0 & 0 & z
\end{array}\right)=\left(\begin{array}{ccc}
0 & z & 0 \\
0 & 0 & z
\end{array}\right)-\left(\begin{array}{ccc}
0 & z & 0 \\
0 & 0 & 0
\end{array}\right) \in T_{M} \mathcal{G}(n)(M) .
$$

Portanto

$$
j^{1} M \sim\left(\begin{array}{ccc}
z & y & 0 \\
0 & x & y
\end{array}\right)
$$

Então

$$
\underline{j^{2} f \sim_{\mathcal{K}}\left(z x, z y, y^{2}\right)} .
$$

Para $b_{1}=0$ ou $b_{2}=0$, temos que:

Se $b_{1}=0$ e $b_{2}=0$

$$
j^{1} M \sim_{\mathcal{G}(n)}\left(\begin{array}{ccc}
y & z & 0 \\
x & 0 & y
\end{array}\right)
$$

Se $b_{1}=0$ e $b_{2} \neq 0$

$$
j^{1} M \sim_{\mathcal{G}(n)}\left(\begin{array}{ccc}
y & z & 0 \\
x & 0 & y
\end{array}\right)
$$

Nestes casos, $j^{2} f \sim_{\mathcal{K}}\left(z x, z y, y^{2}\right)$.

Se $b_{1} \neq 0$ e $b_{2}=0$

$$
j^{1} M \sim_{\mathcal{G}(n)}\left(\begin{array}{ccc}
y & z & 0 \\
z & x & y
\end{array}\right) .
$$

$\operatorname{Assim} \underline{j^{2} f \sim_{\mathcal{K}}\left(z y, y^{2}, z^{2}-x y\right)}$

No caso (a), página (72), temos que:

$$
j^{1} M \sim_{\mathcal{G}(n)}\left(\begin{array}{ccc}
z & y & 0 \\
a_{1} x+b_{1} y+c_{1} z & a_{2} x+b_{2} y+c_{2} z & x
\end{array}\right) .
$$


Suponha que $a_{1} \neq 0$ e $a_{2} \neq 0$.

$$
j^{1} M \sim_{\mathcal{G}(n)}\left(\begin{array}{ccc}
z & y & 0 \\
b_{1} y+c_{1} z & b_{2} y+c_{2} z & x
\end{array}\right) .
$$

Agora suponha que $c_{1} \neq 0$ e $c_{2} \neq 0$

$$
j^{1} M \sim_{\mathcal{G}(n)}\left(\begin{array}{ccc}
c_{2} z-c_{1} y & y & 0 \\
\left(c_{2} b_{1}-b_{2} c_{1}\right) y & b_{2} y+c_{2} z & x
\end{array}\right) \sim_{\mathcal{G}(n)}\left(\begin{array}{ccc}
z & y & 0 \\
\left(c_{2} b_{1}-b_{2} c_{1}\right) y & \tilde{b_{2}} y+\tilde{c_{2} z} & x
\end{array}\right) .
$$

Suponha que $c_{2} b_{1}-b_{2} c_{1}=0$, então:

$$
j^{1} M \sim_{\mathcal{G}(n)}\left(\begin{array}{ccc}
z & y & 0 \\
0 & \tilde{b_{2}} y+\tilde{c_{2}} z & x
\end{array}\right) .
$$

Suponha que $\tilde{b_{2}} \neq 0$

$$
j^{1} M \sim_{\mathcal{G}(n)}\left(\begin{array}{ccc}
z & y & 0 \\
z & z & x
\end{array}\right) \sim_{\mathcal{G}(n)}\left(\begin{array}{ccc}
z & z-y & 0 \\
z & 0 & x
\end{array}\right) \sim_{\mathcal{G}(n)}\left(\begin{array}{ccc}
z & y & 0 \\
z & 0 & x
\end{array}\right) .
$$

Assim

$$
\underline{j^{2} f \sim_{\mathcal{K}}(z x, z y, x y)} .
$$

Os outros casos se reduzem aos casos já feitos anteriormente.

\section{Parte b: Redução do problema à classificação de 3 pontos em $\mathbb{P}^{2}$.}

A classificação feita acima por muitos cálculos de transformações de linhas, colunas e mudanças de coordenadas pode ser reduzida a classificar 3 pontos em $\mathbb{P}^{2}$, página (66). Para isso vamos considerar os seguintes fatos:

Definição 3.13. ([23], página 25) O mergulho de Segre é uma aplicação definida como:

$$
\begin{aligned}
\sigma: \mathbb{P}^{n} \times \mathbb{P}^{m} & \longrightarrow \mathbb{P}^{(n+1)(m+1)-1} \\
\left(\left[x_{0}: \ldots: x_{n}\right],\left[y_{0}: \ldots: y_{m}\right]\right) & \longmapsto\left[x_{0} y_{0}: \ldots: x_{0} y_{1}: \ldots: x_{i} y_{j}: \ldots: x_{n} y_{m}\right]
\end{aligned}
$$

Pode-se verificar que $\sigma$ é uma aplicação injetiva e que a imagem de $\sigma$ é o conjunto de zeros das equações quadráticas $Z_{i j} Z_{k l}-Z_{i l} Z_{k j}$, onde $Z_{i j}$ denota as coordenadas homogêneas de $\mathbb{P}^{(n+1)(m+1)-1}$, ou seja $\sigma\left(\mathbb{P}^{n} \times \mathbb{P}^{m}\right)=V\left(Z_{i j} Z_{k l}-Z_{i l} Z_{k j}\right)$. A imagem é chamada variedade de Segre.

Uma variedade de Segre é um exemplo de uma variedade determinantal. 
1) Uma matriz $M$ de tamanho $3 \times 2$ pode ser considerada como um elemento em $\mathbb{P}^{5}$ (sem considerar a matriz nula). Se todos os menores maximais se anulam, então $M$ é um elemento da imagem do mergulho Segre.

$$
\begin{aligned}
\sigma: \mathbb{P}^{2} \times \mathbb{P}^{1} & \longrightarrow \mathbb{P}^{5} \\
\left(\left[t_{0}: t_{1}: t_{2}\right],\left[s_{0}: s_{1}\right]\right) & \longmapsto\left(\begin{array}{lll}
t_{0} s_{0} & t_{1} s_{0} & t_{2} s_{0} \\
t_{0} s_{1} & t_{1} s_{1} & t_{2} s_{1}
\end{array}\right)
\end{aligned}
$$

Esta imagem é dada pelo ideal $I=\left\langle\left(x_{1} x_{5}-x_{2} x_{4},-x_{1} x_{6}+x_{3} x_{4}, x_{2} x_{6}-x_{3} x_{5}\right)\right\rangle$ e é de grau 3, ver [23] página (234). Portanto, $\mathbb{P}^{2}$ em posição geral intercepta $V(I)$ em três pontos.

2) A matriz $M$ de tamanho $2 \times 3$ com entradas lineares em 3 variáveis, para a qual $\alpha \neq 0$ ou $\alpha=0$ nos casos da parte $(a)$, página (72), define uma inclusão.

$$
\begin{aligned}
& i: \mathbb{P}^{2} \hookrightarrow \hookrightarrow \quad \mathbb{P}^{5} \\
& (x: y: z) \mapsto\left(\begin{array}{ccc}
z & y & M_{13}(x, y, z) \\
M_{21}(x, y, z) & M_{22}(x, y, z) & M_{23}(x, y, z)
\end{array}\right) .
\end{aligned}
$$

3) $\mathbb{P}^{2} \subset \mathbb{P}^{5}$ definida pela inclusão intercepta a imagem do mergulho Segre em um objeto 0-dimensional de multiplicidade 3 (ou a intersecção não é 0dimensional), pelo Teorema de Bézout (ver [24]). Como a imagem da aplicação de Segre é dada por matrizes de tamanho $3 \times 2$, para as quais todos os menores maximais se anulam, o conjunto definido pelos menores maximais de $M$ em $\mathbb{P}^{2}$ consiste de 3 pontos em $\mathbb{P}^{2}$ contando com suas multiplicidades. Assim os seguintes casos podem ocorrer:

- 3 pontos diferentes:

$$
j^{2} f \sim_{\mathcal{K}}(x y, x z, y z) .
$$

- 1 ponto de multiplicidade 2 e um ponto qualquer :

$$
j^{2} f \sim_{\mathcal{K}}\left(z x, z y, y^{2}\right)
$$

- 1 ponto de multiplicidade 3 


$$
\begin{array}{rlll}
j^{2} f & \sim_{\mathcal{K}} & \left(z y, y^{2}, z^{2}-x y\right) \\
\text { e } \quad j^{2} f & \sim_{\mathcal{K}} & \left(z y, z^{2}, y^{2}\right) .
\end{array}
$$

2. Caso 2: $j^{2} f_{i} \neq 0, i \in\{1,2\}, j^{2} f_{3}=0$

Suponha que podemos encontrar uma matriz na órbita de $M$ tal que o 1-jato de alguma entrada não se anula (por exemplo, podemos considerar $j^{1} M_{11} \neq 0$ ), fazendo mudança de coordenadas obtemos:

$$
j^{1} M \sim_{\mathcal{G}(n)}\left(\begin{array}{ccc}
z & L_{12}(x, y) & L_{13}(x, y) \\
L_{21}(x, y) & L_{22}(x, y, z) & L_{23}(x, y, z)
\end{array}\right) .
$$

Usando o fato de que um dos menores maximais se anula e considerando a estrutura da matriz podem ocorrer os casos.

$$
j^{1} M \sim_{\mathcal{G}(n)}\left(\begin{array}{ccc}
z & 0 & 0 \\
L_{21}(x, y) & L_{22}(x, y, z) & L_{23}(x, y, z)
\end{array}\right),
$$

e

$$
j^{1} M \sim_{\mathcal{G}(n)}\left(\begin{array}{ccc}
z & y & 0 \\
L_{21}(x, y) & L_{22}(x, y, z) & L_{23}(x, y, z)
\end{array}\right) .
$$

Considerando o primeiro caso:

$$
j^{1} M \sim_{\mathcal{G}(n)}\left(\begin{array}{ccc}
z & 0 & 0 \\
a_{1} x+b_{1} y & a_{2} x+b_{2} y+c_{2} & a_{3} x+b_{3} y+c_{3} z
\end{array}\right) .
$$

Suponha que $a_{2} \neq 0$ e $b_{3} \neq 0$. Fazendo as seguintes mudanças de coordenadas:

$$
\begin{aligned}
\phi_{1}: \mathbb{C}\{x, y, z\} & \longrightarrow \mathbb{C}\{x, y, z\} \\
x & \longmapsto a_{2} x+b_{2} y+c_{2} \\
y & \longmapsto a_{3} x+b_{3} y+c_{3} z
\end{aligned}
$$

então :

$$
j^{1} M \sim_{\mathcal{G}(n)}\left(\begin{array}{ccc}
z & y & 0 \\
\widetilde{L}_{21}(x, y) & x & y
\end{array}\right) .
$$

Logo $\underline{j^{2} f \sim_{\mathcal{K}}(z x, z y, 0)}$. 
Suponha que

$$
j^{1} M \sim_{\mathcal{G}(n)}\left(\begin{array}{ccc}
z & 0 & 0 \\
a_{1} x+b_{1} y & b_{2} y+c_{2} z & b_{3} y+c_{3} z
\end{array}\right) .
$$

Se $b_{2} \neq 0$ e $b_{3} \neq 0$, fazendo transformações de linhas e colunas temos:

$j^{1} M \sim_{\mathcal{G}(n)}\left(\begin{array}{ccc}z & 0 & 0 \\ a_{1} x+b_{1} y & \left(c_{2} b_{3}-c_{3} b_{2}\right) z & b_{3} y+c_{3} z\end{array}\right) \sim_{\mathcal{G}(n)}\left(\begin{array}{ccc}z & 0 & 0 \\ a_{1} x+b_{1} y & z & b_{3} y+c_{3} z\end{array}\right)$,

e usando a mudança de coordenadas:

$$
\begin{aligned}
\phi_{2}: \mathbb{C}\{x, y, z\} & \longrightarrow \mathbb{C}\{x, y, z\} \\
y & \longmapsto b_{3} y+c_{3} z .
\end{aligned}
$$

Logo

$$
\underline{j^{2} f \sim_{\mathcal{K}}\left(z y, z^{2}, 0\right)} .
$$

Para o segundo caso, pelo fato de ter um maximal igual a zero, temos que:

$$
j^{1} M \sim_{\mathcal{G}(n)}\left(\begin{array}{ccc}
z & y & 0 \\
0 & 0 & a_{3} x+b_{3} y+c_{3} z
\end{array}\right) .
$$

Se $a_{3}=0$, usando a seguinte mudança de coordenadas:

$$
\begin{aligned}
\phi: \mathbb{C}\{x, y, z\} & \longrightarrow \mathbb{C}\{x, y, z\} \\
z & \longmapsto a_{3} x+b_{3} y+c_{3} z,
\end{aligned}
$$

obtemos

$$
j^{1} M \sim_{\mathcal{G}(n)}\left(\begin{array}{ccc}
z & y & 0 \\
0 & 0 & x
\end{array}\right) \sim_{\mathcal{G}(n)}\left(\begin{array}{ccc}
y & x & 0 \\
0 & 0 & z
\end{array}\right)
$$

Portanto:

$$
\underline{j^{2} f \sim_{\mathcal{K}}(z x, z y, 0)} .
$$

Se $a_{3}=0, j^{1} M \sim_{\mathcal{G}(n)}\left(\begin{array}{ccc}z & y & 0 \\ 0 & 0 & b_{3} y+c_{3} z\end{array}\right)$.

Se $c_{3} \neq 0, j^{1} M \sim_{\mathcal{G}(n)}\left(\begin{array}{ccc}z & y & 0 \\ 0 & 0 & b_{3} y+z\end{array}\right)$. 
Usamos o Lema de Mather (1.15) para mostrar que:

$$
\left(\begin{array}{ccc}
z & y & 0 \\
0 & 0 & b_{3} y+z
\end{array}\right) \sim_{\mathcal{G}(n)}\left(\begin{array}{ccc}
z & y & 0 \\
0 & 0 & z
\end{array}\right) \sim_{\mathcal{G}(n)}\left(\begin{array}{ccc}
y & z & 0 \\
0 & 0 & y
\end{array}\right) .
$$

Assim,

$$
\underline{j^{2} f \sim_{\mathcal{K}}\left(z y, y^{2}, 0\right)} .
$$

Se $c_{3}=0, j^{1} M \sim_{\mathcal{G}(n)}\left(\begin{array}{ccc}z & y & 0 \\ 0 & 0 & y\end{array}\right)$.

Logo,

$$
\underline{j^{2} f \sim_{\mathcal{K}}\left(z y, y^{2}, 0\right)} .
$$

3. Caso 3: $j^{2} f_{2}=j^{2} f_{3}=0$

Aqui só existe uma possibilidade: $\underline{j^{2} f \sim_{\mathcal{K}}(q, 0,0)}$.

Pelo Corolário (3.6), página (60) obtemos que $j^{2} f \sim_{\mathcal{K}}(q, 0,0)$ não é simples.

Como $j^{2} f \sim_{\mathcal{K}}\left(z x, z y, z^{2}\right)$ então $j^{1} M \sim_{\mathcal{G}(n)}\left(\begin{array}{lll}0 & z & y \\ y & x & 0\end{array}\right)$. Pelo Corolário 3.8 e pela Tabela (3.1), $f$ não é simples.

\subsection{Passo III: Procurar Candidatos em cada um dos Casos do Item (2).}

Vamos demonstrar um lema para cada um dos casos do passo II. As provas de todos usam as mesmas técnicas e portanto são similares. Para as provas usaremos o Teorema da transversal completa (2.10), Lema de Mather (1.15) e critérios das seções anteriores.

Lema 3.14. Se $j^{2} f \sim_{\mathcal{K}}(z x, z y, x y)$ então $f \sim_{\mathcal{K}}(z x, z y, x y)$.

Demonstração: Temos que

$$
j^{1} M \sim_{\mathcal{G}(n)}\left(\begin{array}{ccc}
z & 0 & y \\
0 & x & y
\end{array}\right) .
$$

Vamos usar o Lema de determinação finita (2.23) para mostrar que $j^{1} M$ é 1-determinado. 
Considere-se o espaço tangente da matriz $M$.

$$
\begin{gathered}
T_{M} \mathcal{G}_{(n)}(M)=\mathbf{m}\left\langle\left(\begin{array}{lll}
0 & 0 & 0 \\
0 & 1 & 0
\end{array}\right),\left(\begin{array}{lll}
0 & 0 & 1 \\
0 & 0 & 1
\end{array}\right),\left(\begin{array}{lll}
1 & 0 & 0 \\
0 & 0 & 0
\end{array}\right)\right\rangle+\mathcal{O}_{3}\left\langle\left(\begin{array}{lll}
z & 0 & y \\
0 & 0 & 0
\end{array}\right),\left(\begin{array}{lll}
0 & 0 & 0 \\
z & 0 & y
\end{array}\right),\right. \\
\left(\begin{array}{lll}
0 & x & y \\
0 & 0 & 0
\end{array}\right)\left(\begin{array}{lll}
0 & 0 & 0 \\
0 & x & y
\end{array}\right),\left(\begin{array}{lll}
z & 0 & 0 \\
0 & 0 & 0
\end{array}\right),\left(\begin{array}{lll}
0 & z & 0 \\
0 & 0 & 0
\end{array}\right),\left(\begin{array}{lll}
0 & 0 & z \\
0 & 0 & 0
\end{array}\right),\left(\begin{array}{lll}
0 & 0 & 0 \\
x & 0 & 0
\end{array}\right) \\
\left.,\left(\begin{array}{lll}
0 & 0 & 0 \\
0 & x & 0
\end{array}\right),\left(\begin{array}{lll}
0 & 0 & 0 \\
0 & 0 & x
\end{array}\right),\left(\begin{array}{lll}
y & 0 & 0 \\
y & 0 & 0
\end{array}\right),\left(\begin{array}{lll}
0 & y & 0 \\
0 & y & 0
\end{array}\right),\left(\begin{array}{lll}
0 & 0 & y \\
0 & 0 & y
\end{array}\right)\right\rangle .
\end{gathered}
$$

Segue-se que

$$
\mathbf{m}^{2} \mathcal{M a t}_{3 \times 2}(\mathbb{C}\{x, y, z\}) \subset \mathbf{m} T_{M} \mathcal{G}(n)(M) .
$$

Então $j^{1} M$ é 1-determinado. $\operatorname{Logo} M \sim_{\mathcal{G}(n)}\left(\begin{array}{ccc}z & 0 & y \\ 0 & x & y\end{array}\right)$. Daí,

$$
f \sim_{\mathcal{K}}(z x, z y, x y)
$$

Lema 3.15. Se $j^{2} f \sim_{\mathcal{K}}\left(z x, z y, y^{2}\right)$ então existe $k \in \mathbb{N}, k \geq 2$ tal que $f \sim_{\mathcal{K}}\left(z x, z y, y^{2}-x^{k+1}\right)$.

\section{Demonstração: Considere}

$$
N \sim_{\mathcal{G}(n)}\left(\begin{array}{ccc}
y & x & 0 \\
0 & y & z
\end{array}\right)
$$

Queremos determinar as órbitas em $J^{2} \mathcal{M} a t_{3 \times 2}(\mathbb{C}\{x, y, z\})$, pela ação de $J^{2} \mathcal{G}(n)$, cujo 1jato seja igual a $N$. Para isto calculamos a transversal completa $T$, assim

$$
T=\left\langle\left(\begin{array}{ccc}
0 & 0 & 0 \\
x^{2} & 0 & 0
\end{array}\right)\right\rangle
$$

Pela Proposição (2.10), segue que $j^{2} M$ é $J^{2} \mathcal{G}(n)$ - equivalente a

$$
\left(\begin{array}{ccc}
y & x & 0 \\
a x^{2} & y & z
\end{array}\right)
$$

Suponha que $a \neq 0$ então:

$$
j^{2} M \sim_{\mathcal{G}(n)}\left(\begin{array}{ccc}
y & x & 0 \\
x^{2} & y & z
\end{array}\right)
$$


como

$$
\left(\begin{array}{ccc}
y & x & 0 \\
x^{2} & y & z
\end{array}\right)
$$

é 2-determinado por Lema (2.23) então

$$
M \sim_{\mathcal{G}(n)}\left(\begin{array}{ccc}
y & x & 0 \\
x^{2} & y & z
\end{array}\right) .
$$

Se $a=0$ podemos considerar o 3 - jato e calcular a transversal completa,

$$
T=\left\langle\left(\begin{array}{ccc}
0 & 0 & 0 \\
b x^{3} & 0 & 0
\end{array}\right)\right\rangle,
$$

no caso em que $b \neq 0$, temos que

$$
j^{3} M \sim_{\mathcal{G}(n)}\left(\begin{array}{ccc}
y & x & 0 \\
x^{3} & y & z
\end{array}\right) .
$$

$\operatorname{como}\left(\begin{array}{ccc}y & x & 0 \\ x^{3} & y & z\end{array}\right)$ é 3-determinado então:

$$
M \sim_{\mathcal{G}(n)}\left(\begin{array}{ccc}
y & x & 0 \\
x^{3} & y & z
\end{array}\right)
$$

Assim para o $k$-jato, usando a transversal completa temos que:

$$
M \sim_{\mathcal{G}(n)}\left(\begin{array}{ccc}
y & x & 0 \\
x^{k} & y & z
\end{array}\right) .
$$

Logo

$$
f \sim_{\mathcal{K}}\left(z x, z y, y^{2}-x^{k+1}\right)
$$

Lema 3.16. Se $j^{2} f \sim_{\mathcal{K}}(z x, z y, 0)$, então $f$ é equivalente a uns dos seguintes casos:

1. $f \sim_{\mathcal{K}}\left(z x, z y, x y^{2}+x^{k-1}\right), \forall k \geq 4$

2. $f \sim_{\mathcal{K}}\left(z x, z y, y^{4}-x^{3}\right)$

3. $f \sim_{\mathcal{K}}\left(z x, z y, x^{3}+x y^{3}\right)$ 
4. $f \sim_{\mathcal{K}}\left(z x, z y, y^{5}-x^{3}\right)$

ou $f$ não pode ser simples pelo Lema (3.7).

Lema 3.17. Se $j^{2} f \sim_{\mathcal{K}}\left(z y, y^{2}, z^{2}-x y\right)$, então $f$ é equivalente a um dos seguintes singularidades:

1. $f \sim_{\mathcal{K}}\left(z y-x^{3}, y^{2}-x^{2} z, z^{2}-x y\right)$

2. $f \sim_{\mathcal{K}}\left(z y+x^{2} z, y^{2}+x^{2} y, z^{2}-x y\right)$

3. $f \sim_{\mathcal{K}}\left(z y-x^{4}, y^{2}-x^{3} z, z^{2}-x y\right)$

ou $f$ não pode ser simples pelo Lema (3.7).

Demonstração: Pela hipótese $j^{1} M=\left(\begin{array}{ccc}z & y & 0 \\ x & z & y\end{array}\right)$.

Ou seja:

$$
M=\left(\begin{array}{ccc}
z+p_{1} & y+p_{2} & p_{3} \\
x+p_{4} & z+p_{5} & y+p_{6}
\end{array}\right)
$$

$\operatorname{com} p_{i} \in \mathbf{m}^{2}$. Consideremos a seguinte mudança de coordenadas:

$$
\begin{aligned}
& \phi: \mathbb{C}\{x, y, z\} \longrightarrow \mathbb{C}\{x, y, z\} \\
& (x, y, z) \longmapsto\left(x+p_{4}, y+p_{2}, z+p_{5}\right) \\
& M \sim_{\mathcal{G}(n)}\left(\begin{array}{ccc}
z+a_{1} & y & a_{2} \\
x & z & y+a_{3}
\end{array}\right), \quad a_{i} \in \mathbf{m}^{2} .
\end{aligned}
$$

Fazendo transformações de colunas podemos eliminar alguns termos de $a_{3} \in \mathbf{m}^{3}$, logo:

$$
M \sim_{\mathcal{G}(n)}\left(\begin{array}{ccc}
z+\tilde{a}_{1} & y & \tilde{a}_{2} \\
x & z & y+p(y)
\end{array}\right), \quad \tilde{a}_{i} \in \mathbf{m}^{2}
$$

onde $p(y)$ é um polinômio em $y$ de grau maior ou igual a 2. Considere-se $p(y)=$ $t_{2} y^{2}+t_{3} y^{3}+\ldots$ Como $1+t_{2} y+t_{3} y^{2} \ldots$ é um elemento invertível, podemos multiplicar este último elemento a uma linha:

$$
M \sim_{\mathcal{G}(n)}\left(\begin{array}{ccc}
\left(z+\tilde{a}_{1}\right)\left(1+t_{2} y+\ldots\right) & y\left(1+t_{2} y+\ldots\right) & \tilde{a}_{2}\left(1+t_{2} y+\ldots\right) \\
x & z & y+p(y)
\end{array}\right), \quad \tilde{a}_{i} \in \mathbf{m}^{2}
$$

e considerando a seguinte mudança de coordenadas:

$$
\begin{aligned}
\phi: \mathbb{C}\{x, y, z\} & \longrightarrow \mathbb{C}\{x, y, z\} \\
y & \longmapsto y\left(1+t_{2} y+\ldots\right),
\end{aligned}
$$


temos que:

$$
M \sim_{\mathcal{G}(n)}\left(\begin{array}{ccc}
z+\widetilde{a_{1}} & y & \widetilde{a_{2}} \\
x & z & y
\end{array}\right), \quad \widetilde{a_{i}} \in \mathbf{m}^{2}
$$

Quando consideramos os pesos do jato da matriz, temos em mente que se cumprem as relações da Definição (2.33), assim $v_{a}(z)-v_{a}(x)=v_{a}(y)-v_{a}(z)$ e que $v_{a}(z)-v_{a}(x)=0$ não fornecem nenhuma contribuição.

1. $j_{(3,5,4)} M$ :

$$
j_{(3,5,4)} M=\left(\begin{array}{ccc}
z & y & \delta_{(3,5,4)} x^{2} \\
x & z & y
\end{array}\right) .
$$

Se $\delta_{(3,5,4)} \neq 0$, por uma mudança de coordenadas temos que:

$$
\begin{aligned}
j_{(3,5,4)} M & \sim_{\mathcal{G}(n)}\left(\begin{array}{ccc}
\sqrt[3]{\delta_{(2,5,4)} z} & \sqrt[3]{\delta_{(2,5,4)}} y & \delta_{(3,5,4)} x^{2} \\
x & \sqrt[3]{\delta_{(2,5,4)} z} & {\sqrt[3]{\delta_{(2,5,4)}} y}^{2}
\end{array}\right) \\
& \sim_{\mathcal{G}(n)}\left(\begin{array}{ccc}
z & y & x^{2} \\
x & z & y
\end{array}\right) .
\end{aligned}
$$

Esta última equivalência se obteve fazendo transformações de linhas e colunas. E $M$ está na mesma órbita de $j_{(3,5,4)} M$, pela Tabela (3.2) e Lema (2.35).

$$
f \sim_{\mathcal{K}}\left(z y-x^{3}, y^{2}-x^{2} z, z^{2}-x y\right)
$$

2. $j_{(2,4,3)} M$

$$
j_{(2,4,3)} M=\left(\begin{array}{ccc}
z & y & -\delta_{(2,4,3)} x z \\
x & z & y
\end{array}\right) .
$$

Se $\delta_{(2,4,3)} \neq 0$, por transformação de coluna, mudança de coordenadas e transformações de linhas e colunas, novamente, obtemos

$$
\begin{aligned}
j_{(2,4,3)} M & \sim_{\mathcal{G}(n)} \\
& \left(\begin{array}{ccc}
z & y & 0 \\
x & z & y+\delta_{(2,3,4)} x^{2}
\end{array}\right) \\
& \sim_{\mathcal{G}(n)}\left(\begin{array}{ccc}
\sqrt{\delta_{(2,3,4)} z} & \delta_{(2,3,4)} y & 0 \\
x & \delta_{(2,3,4)} z & \delta_{(2,3,4)}\left(y+x^{2}\right)
\end{array}\right) \\
& \sim_{\mathcal{G}(n)}\left(\begin{array}{ccc}
z & y & 0 \\
x & z & y+x^{2}
\end{array}\right) .
\end{aligned}
$$


E $M$ está na mesma órbita de $j_{(2,4,3)} M$, pela Tabela (3.2) e Lema (2.35).

$$
f \sim_{\mathcal{K}}\left(z y+x^{2} z, y^{2}+x^{2} y, z^{2}-x y\right)
$$

3. $j_{(3,7,5)} M$ :

$$
j_{(3,7,5)} M=\left(\begin{array}{ccc}
z & y & \delta_{(3,7,5)} x^{3} \\
x & z & y
\end{array}\right) .
$$

Se $\delta_{(3,7,5)} \neq 0$, fazendo uma mudanças de coordenadas considerável e transformações de linha e colunas, obtemos:

$$
\begin{aligned}
j_{(3,7,5)} M & \sim_{\mathcal{G}(n)}\left(\begin{array}{ccc}
\sqrt[3]{\delta_{(3,7,5)} z} & \sqrt[3]{\delta_{(3,7,5)}} y & \delta_{(3,7,5)} x^{3} \\
x & \sqrt[3]{\delta_{(3,7,5)} z} & \sqrt[3]{\delta_{(3,7,5)}} y
\end{array}\right) \\
& \sim \sim_{\mathcal{G}(n)}\left(\begin{array}{ccc}
z & y & x^{3} \\
x & z & y
\end{array}\right) .
\end{aligned}
$$

E $M$ está na mesma órbita de $j_{(3,7,5)} M$, por Tabela (3.2) e Lema (2.35).

$$
f \sim_{\mathcal{K}}\left(z y-x^{4}, y^{2}-x^{3} z, z^{2}-x y\right)
$$

4. $j_{(1,3,2)} M$ :

$$
j_{(1,3,2)} M=\left(\begin{array}{ccc}
z+\delta_{(1,3,2)} x^{2} & y & \tilde{\delta}_{(1,3,2)} x^{4} \\
x & z & y+\tilde{\tilde{\delta}}_{1,3,2} x^{3}
\end{array}\right)
$$

implica que $v_{(D, a)} M \geq 0$ para $a=(1,3,2)$ e $D=\left(\begin{array}{lll}2 & 3 & 4 \\ 1 & 2 & 3\end{array}\right)$.

Além disso $M$ não pode ser simples pelo Corolário (3.8), pois $(D ; a)$ é um dos tipos da Tabela (3.1).

Lema 3.18. Se $j^{2} f \sim_{\mathcal{K}}\left(z y, y^{2}, z^{2}\right)$, então $f$ é equivalente a uns dos seguintes germes:

1. $f \sim_{\mathcal{K}}\left(z y+x^{2} y, y^{2}-x^{2} z, z^{2}+x^{2} z\right)$

2. $f \sim_{\mathcal{K}}\left(z y+x^{2} y, y^{2}-x^{3} z, z^{2}+x^{2} z\right)$

3. $f \sim_{\mathcal{K}}\left(z y-x^{5}, y^{2}-x^{2} z, z^{2}-x^{3} y\right)$

ou $f$ não pode ser simples. 
Lema 3.19. Se $j^{2} f \sim_{\mathcal{K}}\left(z y, y^{2}, 0\right)$ então $f$ é equivalente a uma das curvas seguintes:

1. $f \sim_{\mathcal{K}}\left(z y, y^{2}-x^{3}-x z^{2}, z^{3}+x^{2} z\right)$

2. $f \sim_{\mathcal{K}}\left(z y, y^{2}-x^{3}-x z^{3}, z^{4}+x^{2} z\right)$

3. $f \sim_{\mathcal{K}}\left(z y-x^{3}, y^{2}-x^{2} z, x z^{2}-x^{2} y\right)$

4. $f \sim_{\mathcal{K}}\left(z y-x^{3}, y^{2}-x z^{2}, z^{3}-x^{2} z\right)$

ou $f$ não pode ser simples.

\subsection{Deformações Semi-Universais das Formas Normais}

Nessa seção calculamos algumas deformações universais dos germes classificados na seção anterior.

Teorema 3.20. Todas as curvas espaciais das Tabelas (3.2) e (3.2) são simples.

Definição 3.21. Uma classe de germe $X$ é adjacente a uma outra classe $Y$ e indicamos $X \rightarrow Y$, se qualquer representante $f$ de $X$ pode ser mergulhado em uma deformação $F=\left(u, f_{u}(x)\right)$, $f_{0}=f$ tal que o conjunto $\{u, x\}$ para os quais $f_{u}(x, y) \in Y$ contém $u=x=0$ no seu fecho.

Observação 3.22. Para provar que $f \in X$ é adjacente a $g \in Y, f \rightarrow g$, é suficiente dar uma deformação $f_{t}$ de $f$ tal que $f_{0} \sim f e f_{t} \sim g$ para $t \neq 0$.

Para os candidatos encontrados na seção anterior, consideraremos as deformações semi-universais.

Proposição 3.23. As deformações das formas normais de $A_{k} \vee L e D_{k+2} \vee L$ são as seguintes:

Tipo forma normal deformação Semiuniversal

$$
\begin{aligned}
& A_{k} \vee L \quad\left(\begin{array}{ccc}
z & y & x^{k} \\
0 & x & y
\end{array}\right) \quad\left(\begin{array}{ccc}
z & y+\beta & x^{k}+\gamma+a_{k-1} x^{k-1}+\ldots+a_{1} x \\
\alpha & x & y
\end{array}\right) \\
& D_{k+2} \vee L\left(\begin{array}{ccc}
z & 0 & x^{k}-y^{2} \\
0 & x & y
\end{array}\right) \quad\left(\begin{array}{ccc}
z & \beta+b_{1} y & x^{k}-y^{2}+\gamma+b_{2} y+a_{k-1} x^{k-1}+\ldots+a_{1} x \\
\alpha & x & y
\end{array}\right)
\end{aligned}
$$


Para estas 2 classes vamos listar todas as adjacências explícitas.

1. Para $D_{k+2} \vee L$ temos:

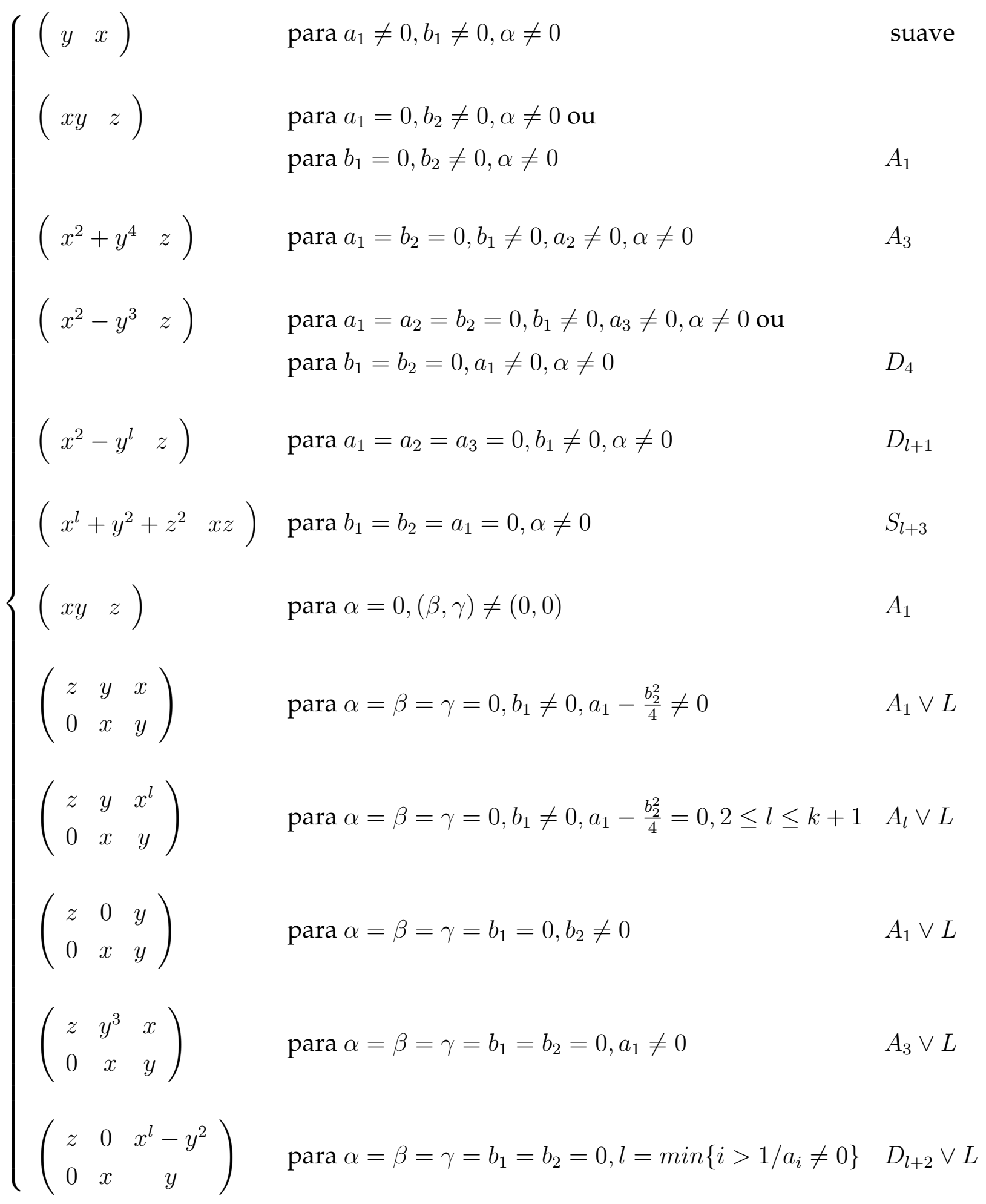


2. Para $A_{k} \vee L$ temos:

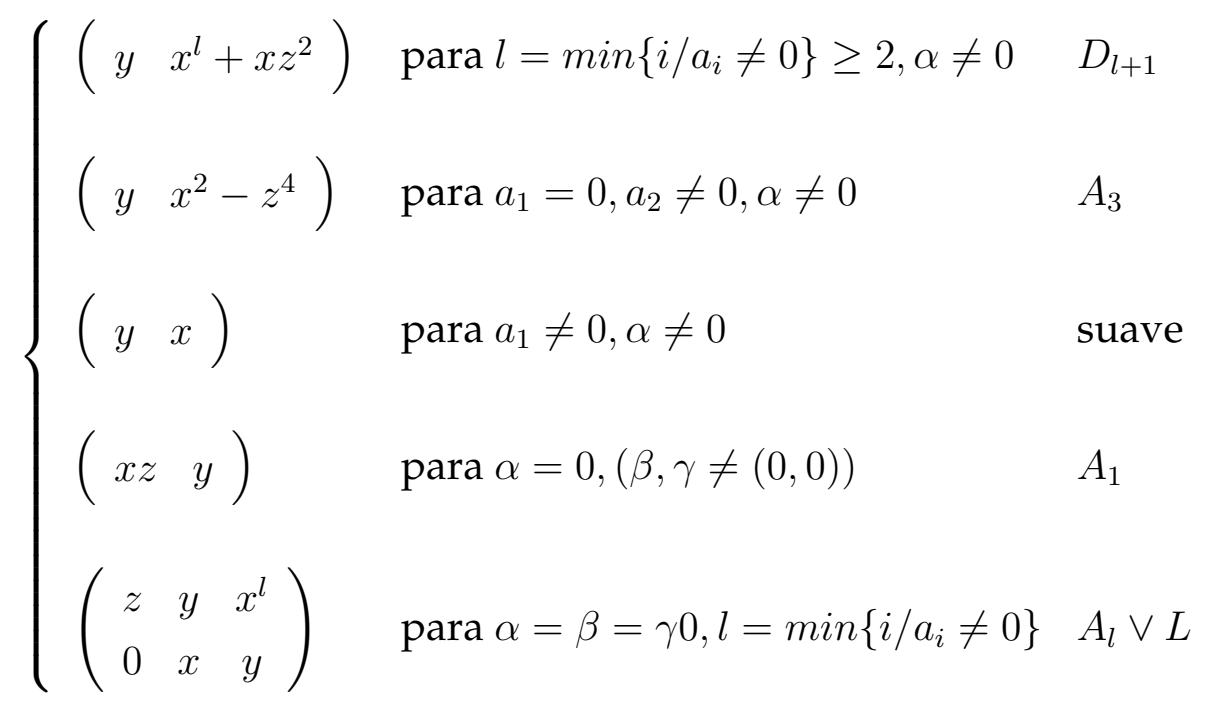

As adjacências $D_{k+2} \vee L \rightarrow A_{k+1} \vee L$ são dadas por $b_{1}=-a_{k-1}, b_{2}=-2 \sqrt{a_{k-1}^{k}}$, $a_{i}=a_{k-1}^{k-i+1} \forall 1 \leq i \leq k-2$.

Como estas singularidades são adjacentes a apenas um múmero finito de singularidades, então as duas classes $A_{k} \vee L$ e $D_{K} \vee L$ são simples.

As perturbações das matrizes descrevendo estas deformações são as seguintes.

$$
\begin{aligned}
& A_{k} \vee L \rightarrow A_{k-1} \vee L \quad\left(\begin{array}{ccc}
z & y & x^{k}+t x^{k-1} \\
0 & x & y
\end{array}\right) \\
& E_{6}(1) \rightarrow A_{2} \vee L \quad\left(\begin{array}{ccc}
z & y & x^{2}+2 t^{3} x \\
x & z & y-3 t^{2} x
\end{array}\right) \\
& E_{7}(1) \rightarrow A_{3} \vee L \quad\left(\begin{array}{ccc}
z & y & -t x^{2}+2 t^{3} x \\
x & z & y+x^{2}-3 t^{2} x
\end{array}\right) \\
& E_{8}(1) \rightarrow A_{4} \vee L \quad\left(\begin{array}{ccc}
z & y & x^{3}-\frac{2}{3} t^{3} x^{2}+\frac{2}{27} t^{6} x \\
x & z & y+2 t x^{2}-\frac{1}{3} t^{4} x
\end{array}\right) \\
& E_{6} \vee L \rightarrow A_{5} \vee L \quad\left(\begin{array}{ccc}
z & y^{2}-t y & x^{3}+2 t x y \\
0 & x & y
\end{array}\right) \\
& E_{7} \vee L \rightarrow A_{6} \vee L \quad\left(\begin{array}{ccc}
z & y^{2}+16 t^{3} y & \frac{40}{3} t^{2} x y+\frac{416}{27} t^{3} x^{2}-\frac{128}{3} t^{4} y-\frac{256}{9} t^{5} x-x^{2} y-\frac{7}{3} t x^{3} \\
0 & x & y
\end{array}\right) \\
& E_{8} \vee L \rightarrow A_{7} \vee L \quad\left(\begin{array}{ccc}
z & y^{2}+t^{5} y & -2 t^{7}+t^{9} x-5 t^{4} x y-4 t x^{2} y+4 t^{6} x^{2}+5 t^{3} x^{3}+x^{4} \\
0 & x & y
\end{array}\right)
\end{aligned}
$$




$$
\begin{aligned}
& E_{12}(2) \rightarrow A_{5} \vee L \quad\left(\begin{array}{ccc}
z+t^{2} x & y+2 t x^{2} & x^{3} \\
x^{2} & z & y
\end{array}\right) \\
& E_{7}(1) \rightarrow E_{6}(1) \quad\left(\begin{array}{ccc}
z & y & t x^{2} \\
x & z & y+x^{2}
\end{array}\right) \\
& E_{8}(1) \rightarrow E_{7}(1) \quad\left(\begin{array}{ccc}
z & y & x^{3} \\
x & z & y+t x^{2}
\end{array}\right) \\
& J_{2,0}(2) \rightarrow E_{7}(1) \quad\left(\begin{array}{ccc}
z+x^{2} & y & x^{2}+t x \\
0 & z & y
\end{array}\right) \\
& J_{2,1}(2) \rightarrow E_{8}(1) \quad\left(\begin{array}{ccc}
z+x^{2} & y & x^{3} \\
t x & z & y
\end{array}\right) \\
& S_{6}^{*} \rightarrow E_{7}(1) \quad\left(\begin{array}{ccc}
z & x & y \\
0 & y & x^{2}-z^{2}-t z
\end{array}\right) \\
& T_{7}^{*} \rightarrow E_{8}(1) \quad\left(\begin{array}{ccc}
z & x & y \\
t x & y & x^{2}-z^{3}
\end{array}\right) \\
& U_{7}^{*} \rightarrow E_{8}(1) \quad\left(\begin{array}{ccc}
z & x & y+\sqrt[3]{9} t^{2} x \\
x^{2}+\sqrt{5} t^{3} x & y & x z+\left(\frac{3 \sqrt{5}}{\sqrt[3]{9}}-\frac{1}{\sqrt[3]{9} \sqrt{5}}\right) t x^{2}-\frac{1}{3}(\sqrt[3]{9})^{2} t^{4} x-\frac{1}{3 \sqrt{5}} t^{3} z
\end{array}\right) \\
& J_{2,1}(2) \rightarrow J_{2,0}(2) \quad\left(\begin{array}{ccc}
z+x^{2} & y & x^{3}+t x^{2} \\
0 & z & y
\end{array}\right) \\
& E_{12}(2) \rightarrow J_{2,1}(2) \quad\left(\begin{array}{ccc}
z & y+3 t^{2} x^{2} & x^{3}+2 t^{3} x^{2} \\
x^{2} & z & y
\end{array}\right) \\
& D_{k} \vee L \rightarrow D_{k-1} \vee L \quad\left(\begin{array}{ccc}
z & 0 & x^{k-2}-y^{2}+x^{k-3} \\
0 & x & y
\end{array}\right) \\
& E_{6} \vee L \rightarrow D_{5} \vee L \quad\left(\begin{array}{ccc}
z & y^{2} & x^{3}+2 t^{3} x^{2}-3 t^{2} x y \\
0 & x & y
\end{array}\right) \\
& E_{7} \vee L \rightarrow D_{6} \vee L \quad\left(\begin{array}{ccc}
z & y^{2}+x^{3} & 3 t^{2} x y-2 t^{3} x^{2}+t x^{3} \\
0 & x & y
\end{array}\right) \\
& E_{8} \vee L \rightarrow D_{7} \vee L \quad\left(\begin{array}{ccc}
z & y^{2} & x^{4}+3 t^{2} x y+2 t^{3} x^{2}-2 \sqrt{3} t x^{2} y-2 t \sqrt{3} t x^{3} \\
0 & x & y
\end{array}\right) \\
& S_{6}^{*} \rightarrow D_{4} \vee L \quad\left(\begin{array}{ccc}
z & x & y \\
t z & y & x^{2}-z^{2}-t^{2} x
\end{array}\right) \\
& T_{7}^{*} \rightarrow D_{5} \vee L \quad\left(\begin{array}{ccc}
z & x & y \\
t z & y & x^{2}-z^{3}+t^{2} x
\end{array}\right) \\
& U_{7}^{*} \rightarrow D_{5} \vee L \quad\left(\begin{array}{ccc}
z & x & y-t^{2} x \\
x^{2} & y & x z-2 t x^{2}
\end{array}\right) \\
& E_{7} \vee L \rightarrow E_{6} \vee L \quad\left(\begin{array}{ccc}
z & y^{2}+x^{3} & t x^{3} \\
0 & x & y
\end{array}\right)
\end{aligned}
$$




$$
\begin{aligned}
& E_{8} \vee L \rightarrow E_{7} \vee L \quad\left(\begin{array}{ccc}
z & y^{2}+t x^{3} & x^{4} \\
0 & x & y
\end{array}\right) \\
& W_{8}^{*} \vee L \rightarrow E_{6} \vee L \quad\left(\begin{array}{ccc}
z & x & y-t^{3} x \\
x^{2} & y & z^{2}+3 t x z+3 t^{2} x^{2}
\end{array}\right) \\
& T_{7}^{*} \rightarrow S_{6}^{*} \quad\left(\begin{array}{ccc}
z & x & y \\
0 & y & x^{2}+z^{3}+t z^{2}
\end{array}\right) \\
& U_{7}^{*} \rightarrow S_{6}^{*} \quad\left(\begin{array}{ccc}
z & x & y \\
x^{2} & y & x z+t x^{2}
\end{array}\right) \\
& W_{8}^{*} \rightarrow T_{7}^{*} \quad\left(\begin{array}{ccc}
z & x & y \\
x^{2} & y & z^{2}+2 t x z+t^{2} x^{2}
\end{array}\right) \\
& W_{8}^{*} \rightarrow U_{7}^{*} \quad\left(\begin{array}{ccc}
z & x & y \\
x^{2} & y & z^{2}+t x z
\end{array}\right)
\end{aligned}
$$

Na sequência apresentaremos o diagrama de adjacências apresentadas por Anne Frühbis-Krüger em [15].

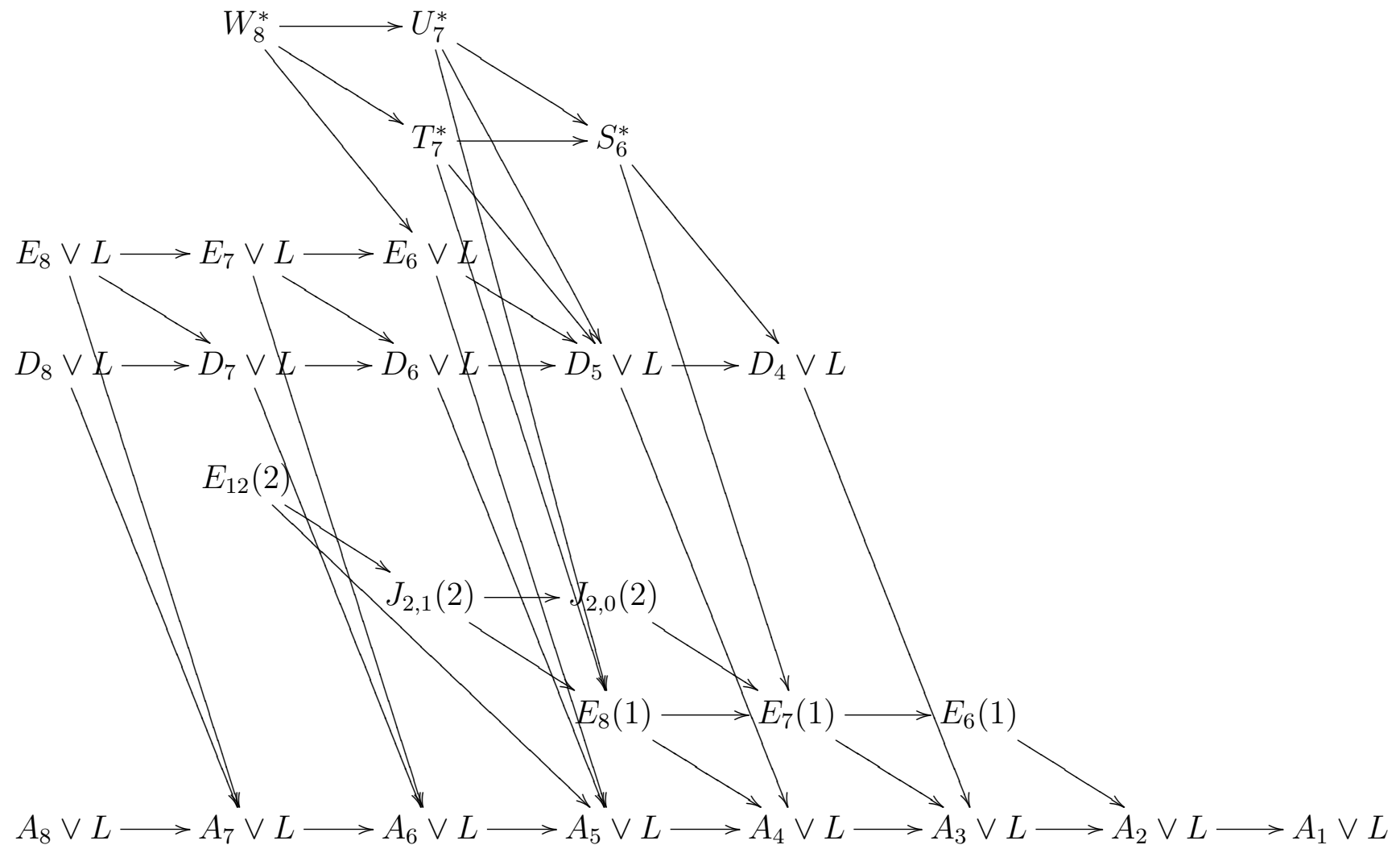

Tabela 3.4: Diagrama de adjacências 


\section{Referências Bibliográficas}

[1] V. I. Arnol'd, S. M. Guseĭn-Zade, and A. N. Varchenko. Singularities of differentiable maps. Vol. I, volume 82 of Monographs in Mathematics. Birkhäuser Boston Inc., Boston, MA, 1985. The classification of critical points, caustics and wave fronts, Translated from the Russian by Ian Porteous and Mark Reynolds.

[2] M. Artin. On the solutions of analytic equations. Invent. Math., 5:277-291, 1968.

[3] M. Artin. Lectures on deformations of singularities. Tata Institute of fundamental Research, Bombay, 1976.

[4] J. W. Bruce. On families of symmetric matrices. Mosc. Math. J., 3(2):335-360, 741, 2003. Dedicated to Vladimir I. Arnold on the occasion of his 65th birthday.

[5] J. W. Bruce and F. Tari. On families of square matrices. Proc. London Math. Soc. (3), 89(3):738-762, 2004.

[6] R.-O. Buchweitz and G.-M. Greuel. The Milnor number and deformations of complex curve singularities. Invent. Math., 58(3):241-281, 1980.

[7] L. Burch. On ideals of finite homological dimension in local rings. Proc. Cambridge Philos. Soc., 64:941-948, 1968.

[8] S. D. Cutkosky and H. Srinivasan. An intrinsic criterion for isomorphism of singularities. Amer. J. Math., 115(4):789-821, 1993.

[9] S. D. Cutkosky and H. Srinivasan. Equivalence and finite determinancy of mappings. J. Algebra, 188(1):16-57, 1997.

[10] M. da Silva Pereira. Variedades Determinantais e Singularidades de Matrizes. Tese de Doutorado, Universidade de São Paulo, ICMC, 2010. 
[11] J. Damon. Deformations of sections of singularities and Gorenstein surface singularities. Amer. J. Math., 109(4):695-721, 1987.

[12] L. R. G. Dias. Singularidades de Matrizes Simétricas. Dissertação de Mestrado, Universidade de São Paulo, ICMC, 2009.

[13] S. Ebey. The classification of singular points of algebraic curves. Trans. Amer. Math. Soc., 118:454-471, 1965.

[14] D. Einsenbud. Commutative Algebra with a view towards Algebraic Geometry. Springer-Verlag, 1995.

[15] A. Frühbis-Krüger. Classification of simple space curve singularities. Comm. Algebra, 27(8):3993-4013, 1999.

[16] A. Frühbis-Krüger. Moduli Spaces for Space Curve Singularities. PhD thesis, Universität Kaiserslautern, 2000.

[17] A. Garcia and Y. Lequain. Elementos de álgebra. Projeto Euclides,IMPA, 2005.

[18] C. G. Gibson. Singular points of smooth mappings, volume 25 of Research Notes in Mathematics. Pitman (Advanced Publishing Program), Boston, Mass., 1979.

[19] C. G. Gibson and C. A. Hobbs. Simple singularities of space curves. Math. Proc. Cambridge Philos. Soc., 113(2):297-310, 1993.

[20] M. Giusti. Classification des singularités isolées simples d'intersections complètes. In Singularities, Part 1 (Arcata, Calif., 1981), volume 40 of Proc. Sympos. Pure Math., pages 457-494. Amer. Math. Soc., Providence, RI, 1983.

[21] G.-M. Greuel, C. Lossen, and E. Shustin. Introduction to singularities and deformations. Springer Monographs in Mathematics. Springer, Berlin, 2007.

[22] G.-M. Greuel and G. Pfister. A Singular introduction to commutative algebra. Springer-Verlag, Berlin, 2002. With contributions by Olaf Bachmann, Christoph Lossen and Hans Schönemann, With 1 CD-ROM (Windows, Macintosh, and UNIX).

[23] J. Harris. Algebraic geometry, volume 133 of Graduate Texts in Mathematics. Springer-Verlag, New York, 1992. A first course.

[24] R. Hartshorne. Algebraic geometry. Springer-Verlag, New York, 1977. Graduate Texts in Mathematics, No. 52. 
[25] H. Hironaka. On the equivalence of singularities. I. In Arithmetical Algebraic Geometry (Proc. Conf. Purdue Univ., 1963), pages 153-200. Harper \& Row, New York, 1965.

[26] J. Martinet. Singularities of smooth functions and maps, volume 58 of London Mathematical Society Lecture Note Series. Cambridge University Press, Cambridge, 1982. Translated from the French by Carl P. Simon.

[27] K. Möhring and D. van Straten. A criterion for the equivalence of formal singularities. Amer. J. Math., 124(6):1319-1327, 2002.

[28] J. J. Rotman. An introduction to homological algebra, volume 85 of Pure and Applied Mathematics. Academic Press Inc. [Harcourt Brace Jovanovich Publishers], New York, 1979.

[29] J. J. Rotman. An introduction to homological algebra. Universitext. Springer, New York, second edition, 2009.

[30] E. Sernesi. Deformations of algebraic schemes, volume 334 of Grundlehren der Mathematischen Wissenschaften [Fundamental Principles of Mathematical Sciences]. Springer-Verlag, Berlin, 2006.

[31] J. Stevens. Deformations of singularities, volume 1811 of Lecture Notes in Mathematics. Springer-Verlag, Berlin, 2003.

[32] C. T. C. Wall. Finite determinacy of smooth map-germs. Bull. London Math. Soc., 13(6):481-539, 1981.

[33] K. Wirthmüller. Torus embeddings and deformations of simple singularities of space curves. Acta Math., 157(3-4):159-241, 1986. 